\title{
Tornehave Morphisms I: Resurrecting the Virtual Permutation Sets Annihilated by Linearization
}

\section{Laurence Barker}

To cite this article: Laurence Barker (2010) Tornehave Morphisms I: Resurrecting the Virtual Permutation Sets Annihilated by Linearization, Communications in Algebra, 39:1, 355-395, DOI: 10.1080/00927870903571855

To link to this article: http://dx.doi.org/10.1080/00927870903571855

$$
\text { 曲 Published online: } 20 \text { Jan } 2011 .
$$

Submit your article to this journal

Џll Article views: 72

Q View related articles $\square$

Citing articles: 1 View citing articles 


\title{
TORNEHAVE MORPHISMS I: RESURRECTING THE VIRTUAL PERMUTATION SETS ANNIHILATED BY LINEARIZATION
}

\author{
Laurence Barker
}

Department of Mathematics, Bilkent University, Bilkent, Ankara, Turkey

Tom Dieck introduced a commutative triangle whereby the exponential morphism from the Burnside functor to the unit functor is factorized through the real representation functor. Tornehave introduced a p-adic variant of the exponential morphism. His construction involves real representations that are not well defined up to isomorphism. To obtain a well defined commutative triangle, we introduce the orientation functor, a quotient of the real representation functor.

Key Words: Burnside functor; Orientation functor; Unit functor; Zombie module.

2000 Mathematics Subject Classification: Primary 19A22; Secondary 20C15.

\section{INTRODUCTION}

To construct a module from a permutation set, the usual device, called linearization, is to regard the permutation set as a basis for the module. We can understand linearization to be a map from the Burnside ring to the character ring. It may seem perverse to try to construct virtual modules from precisely those virtual permutation sets that are killed by the linearization map. Nevertheless, this is what Tornehave successfully did in an unpublished article [17]. His construction is, so to speak, a resurrection only to a shambling form of animation, since his modules are not well defined up to isomorphism. The main aim of the present article is to prove that his modules are well defined up to parity and Galois conjugacy.

The motives for this well-definedness theorem come from the theory of group functors, especially in connection with the Burnside functor $B$, the Burnside unit functor $B^{\times}$, and the real representation functor $A_{\mathbb{R}}$. We draw from a stream of literature that includes Bouc-Yalçın [10], Bouc [8, 9], Yalçın [18]. We also have a view towards two further articles on Tornehave morphisms, [3, 4]. Some of the notation and terminology in the present article is selected for compatibility with those two sequels. For instance, we shall be speaking of the reduced exponential morphism $\overline{\exp }$ and the reduced Tornehave morphism $\overline{\text { torn }}^{\pi}$ because we wish to clearly distinguish them from the lifted exponential morphism exp and the lifted Tornehave morphism torn ${ }^{\pi}$, which will be introduced in [3].

Received December 5, 2008; Revised March 14, 2009. Communicated by J. Zhang.

Address correspondence to Dr. Laurence Barker, Department of Mathematics, Bilkent University, 06800 Bilkent, Ankara, Turkey; E-mail: barker@fen.bilkent.edu.tr 
The reduced exponential morphism $\overline{\exp }: B \rightarrow B^{\times}$can be defined by means of an orbit-counting formula. But it can also be characterized in terms of permutation $\mathbb{R} G$-modules: Tom Dieck [12, 5.5.9] showed that, for a finite group $G$, the reduced exponential map $\overline{\exp }_{G}: B(G) \rightarrow B^{\times}(G)$ factorizes as the composite $\overline{\exp }_{G}=\overline{\operatorname{die}}_{G \text { o }} \operatorname{lin}_{G}$ of the reduced Tom Dieck map $\overline{\operatorname{die}}_{G}: A_{\mathbb{R}}(G) \rightarrow B^{\times}(G)$ and the linearization map $\operatorname{lin}_{G}: B(G) \rightarrow A_{\mathbb{R}}(G)$. The map $\operatorname{die}_{G}$ is defined by a formula which counts dimensions of subspaces fixed by subgroups. The map $\operatorname{lin}_{G}$ is given by $\operatorname{lin}_{G}[X]=[\mathbb{R} X]$, where $[X]$ is the isomorphism class of a finite $G$-set $X$ and $[\mathbb{R} X]$ is the isomorphism class of the permutation $\mathbb{R} G$-module $\mathbb{R} X$. Thus,

$$
\overline{\exp }_{G}[X]=\overline{\operatorname{die}}_{G}[\mathbb{R} X] .
$$

The maps $\overline{\exp }_{G}, \overline{\operatorname{die}}_{G}$, and $\operatorname{lin}_{G}$ commute with induction, restriction, inflation, deflation, and isogation. So we have a commutative triangle of morphisms of biset functors $\overline{\exp }=\overline{\text { die }}_{\mathrm{o}}$ lin.

As a variant of that factorization, we can replace $A_{\mathbb{R}}$ with the biset functor $O_{\mathbb{R}}$, called the orientation functor, which is obtained from $A_{\mathbb{R}}$ by quotienting out modulo parity and modulo Galois conjugacy. When we say that two $\mathbb{C} G$-characters $\chi$ and $\chi^{\prime}$ are Galois conjugate, we mean that $\chi$ and $\chi^{\prime}$ are conjugate under the action of $\operatorname{Aut}(\mathbb{C})$. This is equivalent to the condition that there is a finite-degree Galois extension $\mathbb{K}$ of $\mathbb{Q}$ such that $\chi$ and $\chi^{\prime}$ are conjugate under the action of the Galois group $\operatorname{Gal}(\mathbb{K} / \mathbb{Q})=\operatorname{Aut}(\mathbb{K})$. Thus $O_{\mathbb{R}}(G)=A_{\mathbb{R}}(G) / I(G)$, where $I(G)$ is the $\mathbb{Z}$-submodule of $A_{\mathbb{R}}(G)$ spanned by those $\mathbb{R} G$-characters which can be expressed as the sum $\chi+\chi^{\prime}$ of two Galois conjugate $\mathbb{C} G$-characters $\chi$ and $\chi^{\prime}$. It is not hard to show-see Remark 4.1-that $O_{\mathbb{R}}(G)$ is an elementary abelian 2-group whose rank is the number of Galois conjugacy classes of absolutely irreducible $\mathbb{R} G$ characters. In Section 4, we shall discuss the orientation functor in detail and, in particular, we shall explain the rationale for its name. We have a commutative triangle $\overline{\exp }=\overline{\text { die }}_{\circ} \overline{\text { lin }}$ where the condensed Tom Dieck morphism $\overline{\text { die }}: O_{\mathbb{R}} \rightarrow B^{\times}$ and the condensed linearization morphism $\overline{\operatorname{lin}}: B \rightarrow A_{\mathbb{R}}$ are the morphisms of biset functors induced from $\overline{\mathrm{die}}$ and lin.

Tornehave's construction leads to a kind of $\pi$-adic analogue of those factorizations. Here, $\pi$ is a fixed set of rational primes. Replacing the orbit-counting formula for $\overline{\exp }$ with a formula which adds up the $\pi$-adic valuations of the orbit sizes, we shall define the reduced Tornehave morphism $\overline{\operatorname{torn}}^{\pi}: K \rightarrow B^{\times}$, where $K=$ Ker(lin). Again, the morphism can be characterized in another way. Tornehave showed that, given an element $\kappa \in K(G)$, then

$$
\overline{\operatorname{torn}}_{G}^{\pi}(\kappa)=\overline{\operatorname{die}}[W],
$$

where $W$ is an $\mathbb{R} G$-module associated with $\kappa$ by means of a construction which we shall explain in Section 2. That construction does not yield a factorization of $\overline{\operatorname{torn}}_{G}^{\pi}$ through $A_{\mathbb{R}}(G)$; some arbitrary choices are involved, and $W$ is not well defined up to isomorphism. We call $W$ a zombie module of $\kappa$ because $\operatorname{lin}_{G}$ kills $\kappa$ and because torn $\mathrm{H}_{G}^{\pi}$ only partially resurrects $\kappa$, the module $W$ being ill-defined.

However, subject to a proviso, the image of $W$ in $O_{\mathbb{R}}(G)$ is well defined. The proviso is that, as well as fixing $\pi$, we shall also fix an automorphism $\alpha$ of $\mathbb{C}$ such that $\pi$ is the Kummer symbol of $\alpha$. We mean to say that $\pi$ is the set of primes $p$ 
such that $\alpha(\sqrt{p})=-\sqrt{p}$. A straightforward application of Galois theory and Zorn's Lemma shows that, for any given $\pi$, there exists an $\alpha$ satisfying the hypothesis. We shall introduce a morphism zom ${ }^{\alpha}: B \rightarrow O_{\mathbb{R}}$, and we shall show that $\overline{\operatorname{torn}}^{\pi}$ factorizes as the composite $\overline{\operatorname{torn}}^{\pi}=\overline{\operatorname{die}}_{\mathrm{o}} \mathrm{zom}^{\alpha}$. We conjecture that the proviso is unnecessary, and that zom ${ }^{\alpha}$ depends only on $\pi$.

The three factorizations that we have been discussing are the three commutative triangles depicted. The main theorem in this article, Theorem 2.3, asserts that the third triangle is well defined.
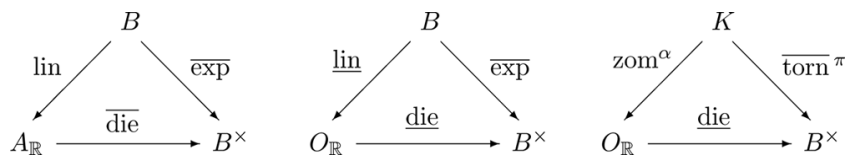

All the morphisms in the first two triangles are morphisms of biset functors; they are Mackey functors, and they also commute with inflation and deflation. The morphisms $\overline{\operatorname{torn}}^{\pi}$ and zom $^{\alpha}$ do not commute with deflation, but they are inflakey morphisms - inflation Mackey morphisms. They are Mackey morphisms which also commute with inflation.

In the next section, we establish some terminology concerning group functors, we state some of our main conclusions, and we indicate some applications that will appear in $[3,4]$. But we postpone to Section 3 some details of the definitions. In Sections 4 to 8 , we discuss a topological interpretation of the orientation functor $O_{\mathbb{R}}$, and then we present the proof of the main theorem. Each of the examples discussed in Sections 9-12 has some general theoretical significance. Letting $D_{m}$ denote the dihedral group with order $m$, we shall examine the case $G=D_{2 p}$ to show that $\overline{\operatorname{torn}}^{\pi} \neq \overline{\operatorname{torn}}^{{ }^{\prime}}$ when $\pi \neq \pi^{\prime}$. We shall examine the case $G=D_{2^{n}}$ to exhibit a surprising phenomenon concerning Galois conjugacy.

\section{CONCLUSIONS}

Like almost all mathematical stories, this one begins with material that is already known to experts. This section narrates first the background and then the statements of the main results of this article. Technical details requiring a larger foundation of notation-for instance, some of the defining formulas-are deferred to Section 3.

Tornehave's original application of the map $\overline{\operatorname{torn}}_{G}^{\pi}$ is related to the following theorem which, as we shall explain in Section 5, is equivalent to Bouc $[8,9.5,9.6]$.

Theorem 2.1 (Bouc). If $G$ is nilpotent, then the condensed Tom Dieck map $\overline{\operatorname{die}}_{G}$ : $O_{\mathbb{R}}(G) \rightarrow B^{\times}(F)$ is an $\mathbb{F}_{2}$-linear isomorphism. In other words, treating $O_{\mathbb{R}}$ and $B^{\times}$as biset functors for the class of nilpotent groups, then the condensed Tom Dieck morphism $\overline{\mathrm{die}}: O_{\mathbb{R}} \rightarrow B^{\times}$is an isomorphism of biset functors.

What Tornehave proved [17] was the surjectivity of $\overline{\operatorname{die}}_{G}$ for nilpotent $G$. Another proof of the surjectivity property was given by Yalçın [18, 1.1]. Bouc proved the injectivity property of $\overline{\operatorname{die}}_{G}$ and he also recognized that the theory of 
biset functors provides an illuminating setting for line of argument that Yalçın had presented.

But the proof originally given by Tornehave is still of interest. In view of the strange defining formulas for $\overline{\operatorname{torn}}^{\pi}$ and $\operatorname{torn}^{\pi}$, even their morphism properties are surprising, let alone the fundamental uniqueness properties of the morphism $\operatorname{torn}^{p}=\operatorname{torn}^{\{p\}}$ that are proved in [3]. Eventually, in [4], it is shown that $\overline{\operatorname{torn}}^{p}$ induces an isomorphism of Bouc [7, 6.5] whereby a difference between rhetorical biset functors and rational biset functors is related to a difference between real representation theory and rational representation theory. As a $p$-biset functor-we mean, a biset functor for the class of finite $p$-groups-let ${ }^{\mathbb{Q}} K$ be the subfunctor of $K$ generated by the coordinate module at the dihedral group with order 8 or the extra-special group with order $p^{3}$. As explained in $[9,5.3,5.6], K$ and ${ }^{\mathbb{Q}} K$ are related to rhetorical $p$-biset functors and rational $p$-biset functors, respectively. On the other hand, by Theorem 2.1-actually, just by the surjectivity property proved by Tornehave-we have $B=\operatorname{die}\left(A_{\mathbb{R}}\right)$ for $p$-groups. Letting ${ }^{\mathbb{Q}} B=\operatorname{die}\left(A_{\mathbb{Q}}\right)$, where $A_{\mathbb{Q}}$ denotes the rational representation functor, then $B$ and ${ }^{\mathbb{Q}} B$ are evidently related to real representations and rational representations, respectively. The main aim of [4] is to show that Bouc's apparently mysterious isomorphism of $p$-biset functors

$$
K /{ }^{\mathbb{Q}} K \cong B^{\times} /{ }^{\mathbb{Q}} B^{\times}
$$

is not just a miraculous coincidence: it commutes with torn ${ }^{p}$ via the canonical epimorphisms $K \rightarrow K /{ }^{\mathbb{Q}} K$ and $B^{\times} \rightarrow B^{\times} /{ }^{\mathbb{Q}} B$. The proof of that commutativity theorem makes use of results in [3] combined with some results that first appeared in Tornehave's proof of the surjectivity property.

The present article, though, is concerned with the problem of factorizing $\overline{\operatorname{torn}}^{\pi}$ through a suitable quotient biset functor of $A_{\mathbb{R}}$. In view of Theorem 2.1, it is reasonable to propose that this quotient biset functor should be $O_{\mathbb{R}}$, at least in the case of nilpotent groups. The difficulty is in proving that the zombie modules yield well defined elements of $O_{\mathbb{R}}$ for arbitrary finite groups. Actually, the main results in $[3,4]$ do not require us to deal with this problem. Our interest in the matter derives from a curiosity as to how the purely algebraic features of $\overline{\operatorname{torn}}^{\pi}$ discussed in [3, 4] are related to the role of the Burnside unit group $B^{\times}(G)$ in the study of $G$-spheres.

Let us be clear about the various kinds of group functors that we shall be considering. The theory of biset functors was introduced by Bouc [6]. We shall employ some notation and terminology that appears in an introductory account [2, Section 2]. Briefly, a biset functor $L$ for a suitable class of groups $\mathscr{X}$ consists of a coordinate module $L(G)$ for each $G$ in $\mathscr{X}$; furthermore, $L$ is equipped with five kinds of maps between the coordinate modules. These five kinds of maps, called the elemental maps, are the induction map $\operatorname{ind}_{G, H}: L(H) \rightarrow L(G)$, the restriction map $\operatorname{res}_{H, G}: L(G) \rightarrow L(H)$, the inflation map $\inf _{G, G / N}: L(G / N) \rightarrow L(G)$, the deflation

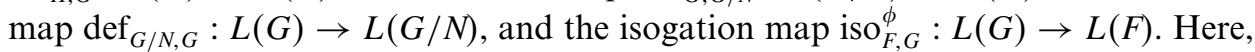
$H \leq G \unrhd N$ and $\phi$ is a group isomorphism $G \stackrel{\sim}{\rightarrow} F$. The elemental maps are required to satisfy certain commutation relations, which we shall discuss in a moment. A morphism of biset functors $\theta: L \rightarrow L^{\prime}$ consists of coordinate maps $\theta_{G}: L(G) \rightarrow$ $L^{\prime}(G)$ which commute with the elemental maps. When the coordinate modules $L(G)$ are modules of a commutative unital ring $R$ and the elemental maps are $R$-module 
homomorphisms, we call $L$ a biset functor over $R$. For biset functors over $R$, the morphisms are required to be $R$-module homomorphisms.

The full list of all fifteen commutation relations for the elemental maps has never been recorded in print. This is because there are some other well-known and more useful characterizations of the notion of a biset functor. Bouc [6] defined a biset functor for $\mathscr{X}$ over $R$ to be an $R$-additive morphism $R \mathscr{C}^{\mathscr{X}} \rightarrow R$-Mod, we mean to say, from the biset category for $\mathscr{X}$ over $R$ to the category of $R$-modules. Equivalently, as in [2], the biset functors for $\mathscr{X}$ over $R$ can be regarded as the locally unital modules of an $R$-algebra $R \Gamma^{\mathscr{L}}$, called the alchemic algebra, which is the $R$ algebra generated by (abstract forms of) the elemental maps.

We shall also be working with some other kinds of group functors. Generally, we understand a group functor for $\mathscr{X}$ over $R$ to be an $R$-additive functor $R \mathscr{D}^{\mathscr{L}} \rightarrow$ $R$-Mod, where $R \mathscr{D}^{\mathscr{X}}$ is a subcategory of $R \mathscr{C}^{\mathscr{X}}$ owning all the isogation morphisms. Equivalently, we can understand a group functor for $\mathscr{X}$ over $R$ to be a locally unital $R \Delta^{\mathscr{L}}$-module, where $R \Delta^{\mathscr{X}}$ is a subalgebra of $R \Gamma^{\mathscr{L}}$ owning all the isogation maps. Our discussions will involve all five of the following particular cases. We define an isogation functor to be a collection of coordinate modules equipped with isogation maps; this is the case where $R \mathscr{D}^{\mathscr{X}}$ is generated by the isogation morphisms, or equivalently, $R \Delta^{\mathscr{L}}$ is generated by the isogation maps. An induction functor is an isogation functor that is also equipped with induction maps. A Mackey functor is an induction functor equipped also with restriction maps. An inflaky functor is a Mackey functor equipped also with inflation maps. Thus, a biset functor is an inflaky functor equipped also with deflation maps. Actually, the group functors that we shall be considering will always be biset functors, but we shall be working with various kinds of morphism: isogation morphisms, induction morphisms, Mackey morphisms, inflaky morphisms, and morphisms of biset functors.

Let us specify Tornehave's construction of the zombie modules. Some proofs will be needed to confirm the viability of the steps, and some further notation would be helpful for the sake of clarity, but let us postpone such details to Section 3 . Recall the we have fixed an automorphism $\alpha \in \mathbb{C}$ with Kummer symbol $\pi$. Now, any element $\kappa \in K(G)$ can be expressed as a formal difference $\kappa=[X]-[Y]$, where $X$ and $Y$ are $G$-sets whose corresponding real permutation modules $\mathbb{R} X$ and $\mathbb{R} Y$ are isomorphic to each other. Taking $X$ and $Y$ to be orthonormal bases of $\mathbb{R} X$ and $\mathbb{R} Y$, it can be shown that there exists a $G$-invariant isometry between $\mathbb{R} X$ and $\mathbb{R} Y$. Identifying $\mathbb{R} X$ with $\mathbb{R} Y$ via a $G$-invariant isometry, and then passing to the complexification $\mathbb{C} X=\mathbb{C} Y$, we let $\theta$ be the composite operator on $\mathbb{C} X$ formed by first applying $\alpha^{-1}$ to the coordinates associated with $X$ and then applying $\alpha$ to the coordinates associated with $Y$. It can be shown that $\theta$ is a $G$-invariant orthogonal operator. In the group of such operators, we can deform $\theta$ to the $\mathbb{C}$-linear extension of an operator $\theta_{\mathbb{R}}$ on $\mathbb{R} X$. The $(-1)$-eigenspace $W$ of $\theta_{\mathbb{R}}$ is an $\mathbb{R} G$-submodule of $\mathbb{R} X$. We call $W$ a zombie module for $\kappa$ (with respect to $\alpha$ ).

Tornehave [17, 1.2] obtained the following result, which we shall recover in Section 7. He used it to confirm that, taking his $\pi$-adic orbit-counting formula as the definition of $\overline{\operatorname{torn}}_{G}^{\pi}$, then $\overline{\operatorname{torn}}_{G}^{\alpha}(\kappa) \in B^{\times}(G)$, hence $\overline{\operatorname{torn}}_{G}^{\pi}$ is well defined as a map $K(G) \rightarrow B^{\times}(G)$.

Proposition 2.2 (Tornehave). With the notation above, $\overline{\operatorname{die}}_{G}[W]=\overline{\operatorname{torn}}_{G}^{\pi}(\kappa)$. 
Alas, for fixed $\alpha$ and $\kappa$, the zombie module $W$ is not well defined up to isomorphism. That is to say, the element $[W]$ of $A_{\mathbb{R}}(G)$ is not well defined. In Section 11 , we shall show that, given a zombie module $W$ for $\kappa$ and any $\mathbb{R} G$-module $M$, then $W \oplus M \oplus M$ is a zombie module for $W$. Nor is the image of $[W]$ in $\mathbb{F}_{2} A_{\mathbb{R}}(G)$ well defined. In the same section, we shall exhibit a counterexample in the case $G=D_{2^{n}}$ with $n \geq 4$. However, in Section 8 , we shall prove that the image of $[W]$ in $O_{\mathbb{R}}(G)$ is well defined. That will quickly lead to the following theorem.

Theorem 2.3. There is a well defined inflaky morphism zom ${ }^{\alpha}: K \rightarrow O_{\mathbb{R}}$ such that, letting $W$ be any zombie module for an element $\kappa \in K(G)$, then $\operatorname{zom}_{G}^{\alpha}(\kappa)$ is the image of $[W]$ in $O_{\mathbb{R}}(G)$. Furthermore, $\overline{\operatorname{torn}}^{\pi}={\overline{\operatorname{die}_{\circ}}}_{\circ} \mathrm{zom}^{\alpha}$.

For finite nilpotent groups, Theorem 2.3 follows immediately from Theorem 2.1 and Proposition 2.2. For arbitrary finite groups, though, that argument collapses because the conclusion of Theorem 2.1 no longer holds. Indeed, in Section 5, we shall give examples to show that, for arbitrary finite groups, die is neither an epimorphism nor a monomorphism.

I worry that some readers may be disquieted by the sometimes formulaic mode of this article, especially in the adaptations of Dirac notation and in the use of matrices and coordinates. A fully structuralistic (or "conceptual") treatment of the results would require different arguments (and would, therefore, be of considerable interest).

\section{CONSTRUCTIONS AND DEFINITIONS}

We now give a more detailed account of the constructions involved in the three commutative triangles depicted in Section 1.

Some arithmetical notation will be needed. We write par() to denote the unique group isomorphism from the field $\mathbb{F}_{2}$ to the unit group $\mathbb{Z}^{\times}=\{ \pm 1\}$. Thus, $\operatorname{par}(0)=1$ and $\operatorname{par}(1)=-1$. Abusing notation, we also write $\operatorname{par}()$ to denote the unique group epimorphism $\mathbb{Z} \rightarrow \mathbb{Z}^{\times}$. Thus $\operatorname{par}(n)=(-1)^{n}$ for an integer $n$. Supposing now that $n>0$, and writing $n=p_{1} \ldots p_{r}$ as a product of primes, the $\pi$ adic valuation of $n$ is defined to be $\log _{\pi}(n)=\left|\left\{i: p_{i} \in \pi\right\}\right|$. Eventually, in Section 7, we shall see that Proposition 2.2 and the commutativity of the third triangle derive, in some sense, from the arithmetical relation

$$
\alpha(\sqrt{n})=\operatorname{par}\left(\log _{\pi}(n)\right) \sqrt{n} .
$$

The first triangle, expressing the equality $\overline{\exp }=\overline{\text { die }}_{\mathrm{o}}$ lin, is discussed in Yoshida [19] and Yalçın [18]. Let us review a few features that we shall be needing later. Recall that the Burnside algebra $\mathbb{Q} B(G)$ has a $\mathbb{Q}$-basis consisting of the primitive idempotents. The ghost ring $\beta(G)$ is defined to be the $\mathbb{Z}$-submodule of $\mathbb{Q} B(G)$ spanned by the primitive idempotents. Obviously, $\beta(G)$ is a subring of $\mathbb{Q} B(G)$. The ghost unit group $\beta^{\times}(G)$ is defined to be the unit group of $\beta(G)$.

The set of primitive idempotents of $\mathbb{Q} B(G)$ can be written as $\left\{e_{I}^{G}: I \leq_{G} G\right\}$, where the notation indicates that $I$ runs over representatives of the conjugacy classes of subgroups of $G$. The $I$ th primitive idempotent $e_{I}^{G}$ is the unique primitive idempotent that is not annihilated by the algebra map $\epsilon_{I}^{G}: \mathbb{Q} B(G) \rightarrow \mathbb{Q}$ given by 
$[X] \mapsto\left|X^{I}\right|$. Here, we are writing $X^{I}$ to denote the set of $I$-fixed points of a $G$-set $X$. Gluck's Idempotent Formula [14] is

$$
e_{I}^{G}=\frac{1}{\left|N_{G}(I)\right|} \sum_{U \leq I}|U| \mu(U, I)[G / U],
$$

where $\mu$ is the Möbius function for the poset of subgroups of $G$. Any element $x \in$ $\mathbb{Q} B(G)$ has coordinate decomposition

$$
x=\sum_{I \leq G} \epsilon_{I}^{G}(x) e_{I}^{G},
$$

where, again, the notation indicates that $I$ runs over representatives of the conjugacy classes of subgroups of $G$. We have $x \in \beta(G)$ if and only if each $\epsilon_{I}^{G}(x) \in \mathbb{Z}$. Also, $x \in$ $\beta^{\times}(G)$ if and only if each $\epsilon_{I}^{G}(x) \in \mathbb{Z}^{\times}$. Plainly, $B(G) \leq \beta(G)$ and $B^{\times}(G) \leq \beta^{\times}(G)$.

It will be convenient to switch from this multiplicative notation to an additive notation. We regard $\beta^{\times}(G)$ as an $\mathbb{F}_{2}$-vector space with basis $\left\{\partial_{I}^{G}: I \leq_{G} G\right\}$, where $\partial_{I}^{G}=1-2 e_{G}^{G}$. When $x \in \beta^{\times}(G)$, we write

$$
x=\sum_{I \leq G}[x @ I] \partial_{I}^{G}
$$

with $[x @ I] \in \mathbb{F}_{2}$. The multiplicative notation and the additive notation for the coordinate decomposition of an element $x \in \beta^{\times}(G)$ are related to each other by the equation

$$
\epsilon_{I}^{G}(x)=\operatorname{par}[x @ I]
$$

The notation here, an adaptation of Dirac notation, is developed systematically in [3]. We can read [x@I] as: the value of $x$ at $I$.

Let us recall the definition of the reduced exponential map

$$
\overline{\exp }_{G}: B(G) \rightarrow B^{\times}(G)
$$

Any element of $B(G)$ can be written in the form $[X]-[Y]$, where $[X]$ and $[Y]$ denote the isomorphism classes of (finite) $G$-sets $X$ and $Y$. We define the element $\overline{\exp }_{G}([X]-[Y]) \in \beta^{\times}(G)$ to be such that the $I$ th coordinate $\left[\overline{\exp }_{G}([X]-[Y]) @ I\right] \in$ $\mathbb{F}_{2}$ is zero if and only if the number of $I$-orbits in $X$ has the same parity as the number of $I$-orbits in $Y$. Let us write $f \stackrel{\text { par }}{=} n$ when an element $f \in \mathbb{F}_{2}$ is the modulo 2 reduction of an element $n \in \mathbb{Z}$. Then

$$
\left[\overline{\exp }_{G}([X]-[Y]) @ I\right] \stackrel{\text { par }}{=}|I \backslash X|-|I \backslash Y|,
$$

where $I \backslash X$ denotes the set of $I$-orbits in $X$.

The latest formula realizes $\overline{\exp }_{G}$ as a well defined linear map with codomain $\beta^{\times}(G)$. To realize $\overline{\exp }_{G}$ as a linear map with codomain $B^{\times}(G)$, one must confirm that the image $\overline{\exp }_{G}(B(G))$ is contained in $B^{\times}(G)$. This is, of course, very well 
known, but the argument will be relevant to our later discussions, so let us recall it. The first step of the argument is to define the reduced Tom Dieck map

$$
\overline{\operatorname{die}}_{G}: A_{\mathbb{R}}(G) \rightarrow B^{\times}(G) .
$$

Any element of $A_{\mathbb{R}}(G)$ can be written in the form $[U]-[V]$, where $[U]$ and $[V]$ are the isomorphism classes of (finite-dimensional) $\mathbb{R} G$-modules $U$ and $V$. As an element of $\beta^{\times}(G)$, we define $\overline{\operatorname{die}}_{G}([U]-[V])$ to be such that the $I$ th coordinate $\left[\overline{\operatorname{die}}_{G}([U]-[V]) @ I\right] \in \mathbb{F}_{2}$ is zero if and only if the dimension of the $I$-fixed subspace $U^{I}$ has the same parity as the dimension of the $I$-fixed subspace $V^{I}$. That is to say,

$$
\left[\overline{\operatorname{die}}_{G}([U]-[V]) @ I\right] \stackrel{\text { par }}{=} \operatorname{dim}_{\mathbb{R}}\left(U^{I}\right)-\operatorname{dim}_{\mathbb{R}}\left(V^{I}\right)
$$

As before, the formula realizes $\overline{\operatorname{die}}_{G}$ as a well defined map with codomain $\beta^{\times}(G)$. Using a topological argument, Tom Dieck [12, 5.5.9] showed that $\overline{\operatorname{die}}_{G}(B(G)) \leq$ $B^{\times}(G)$. So we can understand $\overline{\operatorname{die}}_{G}$ to be a map with codomain $B^{\times}(G)$. Finally, from the defining formulas for $\overline{\exp }_{G}$ and $\overline{\operatorname{die}}_{G}$, an easy calculation yields $\overline{\exp }_{G}=\overline{\operatorname{die}}_{G \circ} \operatorname{lin}_{G}$. This completes the confirmation that $\overline{\exp }_{G}(B(G)) \leq B^{\times}(G)$. Thus, we have realized $\overline{\exp }_{G}$ as a map with codomain $B^{\times}(G)$.

Let us make some brief comments on how the coordinate modules $B(G)$ and $A_{\mathbb{R}}(G)$ give rise to biset functors $B$ and $A_{\mathbb{R}}$. Full definitions of $B$ and $A_{\mathbb{R}}$ are given in Bouc [8, 3.2] and [6, 10.1], respectively. Explicit formulas for the elemental maps for $B$ and $A_{\mathbb{R}}$ can be found in Yalçın [18, Section 3], Yoshida [19, Sections 2, 3]. All we shall be needing are the following observations. Given a subgroup $H \leq G$, then the induction maps $\operatorname{ind}_{G, H}: B(H) \rightarrow B(G)$ and $\operatorname{ind}_{G, H}: A_{\mathbb{R}}(H) \rightarrow A_{\mathbb{R}}(G)$ come from the classical induction operations $G \times_{H}$ and $\mathbb{R} G \otimes_{\mathbb{R} H}$ which send $H$-sets and $\mathbb{R} H$-modules to $G$-sets and $\mathbb{R} G$-modules. The restriction maps $\operatorname{res}_{H, G}$ on $B$ and on $A_{\mathbb{R}}$ come similarly from the operations $G \times_{G}$ and $\mathbb{R} G \otimes_{\mathbb{R} G}$ (with $H$ acting by left translation on $G$ and $\mathbb{R} G$ ). Given a normal subgroup $N \unlhd G$, then inflation $\inf _{G, G / N}$ arises by letting $G$ act on $G / N$-sets and on $\mathbb{R} G / N$-modules via the canonical epimorphism $G \rightarrow G / N$. Deflation $\operatorname{def}_{G / N, G}$ arises by replacing a $G$-set $X$ with the set of $N$-orbits $N \backslash X$ and by replacing an $\mathbb{R} G$-module $M$ with the $N$-fixed subspace $M^{N}$. Given a group isomorphism $\phi: G \stackrel{\sim}{\rightarrow} F$, then isogation iso $_{F, G}^{\phi}$ arises by letting $F$ act on $G$-sets and on $\mathbb{R} G$-modules via $\phi$. It is easy to see that, letting $G$ vary, then the $\operatorname{maps} \operatorname{lin}_{G}$ commute with the five elemental maps. In other words, the maps $\operatorname{lin}_{G}$ are the coordinate maps of a morphism of biset functor lin : $B \rightarrow A_{\mathbb{R}}$.

The definitions of $B^{\times}$and $\beta^{\times}$, as biset functors, are rather more complicated. They are discussed in Bouc [8, Sections 5, 7], Yalçın [18, Section 3], Yoshida [19, Sections 2, 3]; see also a review in [3, Section 10]. For the purposes of the present article, though, we need invoke only Yoshida's result [19, 3.5], which asserts that the maps $\overline{\operatorname{die}}_{G}$ commute with the five elemental maps. Hence, as observed in $[19,3.6]$, the maps $\overline{\exp }_{G}=\overline{\operatorname{die}}_{G \circ} \operatorname{lin}_{G}$ commute with the five elemental maps. Thus we obtain morphisms of biset functors $\overline{\text { die }:} A_{\mathbb{R}} \rightarrow B^{\times}$and $\overline{\exp }: B \rightarrow B^{\times}$. Having now given a complete account of those two morphisms, we have now fully established the first and second of the three commutative triangles depicted in Section 1. 
Our main concern, though, is with the third triangle. The reduced Tornehave map

$$
\overline{\operatorname{torn}}_{G}^{\pi}: K(G) \rightarrow B^{\times}(G)
$$

is defined as follows. Consider an element $\kappa \in K(G)$ and, as before, write $\kappa=$ $[X]-[Y]$ where $X$ and $Y$ are $G$-sets. The hypothesis on $\kappa$ implies that $\mathbb{R} X \cong \mathbb{R} Y$. As an element of $\beta^{\times}(G)$, we define $\overline{\operatorname{torn}}_{G}^{\pi}(\kappa)$ to be such that the Ith coordinate $\left[\overline{\operatorname{torn}}_{G}(\kappa) @ I\right] \in \mathbb{F}_{2}$ is zero if and only if, up to parity, $X$ and $Y$ have the same number of $I$-orbits $\mathscr{O}$ with $\log _{\pi}|\mathscr{O}|$ odd. That is,

$$
\left[\overline{\operatorname{torn}}_{G}^{\pi}(\kappa) @ I\right] \stackrel{\text { par }}{=} \sum_{\mathscr{G} \in I \backslash X} \log _{\pi}|\mathscr{O}|-\sum_{\mathscr{O} \in I \backslash Y} \log _{\pi}|\mathscr{O}| .
$$

Once again, the formula specifies a map with codomain $\beta^{\times}$.

To realize $\overline{\operatorname{torn}}_{G}^{\pi}$ as a map with codomain $B^{\times}(G)$, and also to realize $\overline{\operatorname{torn}}^{\pi}$ as an inflaky morphism, we shall argue as we did above for $\overline{\exp }_{G}$. In place of the morphism of biset functors lin, we shall be making use of the inflaky morphism zom $^{\alpha}$. We shall eventually get back to this argument in Proposition 7.2, when we shall have shown that zom ${ }^{\alpha}$ is well defined.

Before defining the zombie map $\operatorname{zom}_{G}^{\alpha}$, we need the following lemma. It is well known, but the author has been unable to locate a full proof of it in the literature.

Lemma 3.1. Let $U$ and $V$ be mutually isomorphic (finite-dimensional) $\mathbb{R} G$-modules equipped with $G$-invariant inner products. Then there exists a $G$-invariant isometry between $U$ and $V$.

Proof. We may assume that $U=V=\mathbb{R}^{n}$ as $\mathbb{R} G$-modules and that $G$ acts as orthogonal operators on $\mathbb{R}^{n}$. Let $\langle-\mid-\rangle$ be the standard inner product on $\mathbb{R}^{n}$, and let $\langle-\mid-\rangle^{\prime}$ be another $G$-invariant inner product on $\mathbb{R}^{n}$. We are required to show that there exists a $G$-invariant invertible matrix $R$ such that $\langle u \mid v\rangle^{\prime}=\langle R u \mid R v\rangle$ for all $u, v \in \mathbb{R}^{n}$. Writing $u^{T}$ to denote the transpose of $u$, then $\langle u \mid v\rangle=u^{T} v$ and $\langle u \mid v\rangle^{\prime}=u^{T} S v$ where $S$ is a $G$-invariant invertible symmetric matrix. Since $S$ is diagonalizable with strictly positive eigenvalues, there exists a $G$-invariant invertible symmetric matrix $R$ such that $R^{2}=S$. We have $\langle u \mid v\rangle^{\prime}=u^{T} R^{2} v=(R u)^{T} R v=$ $\langle R u \mid R v\rangle$.

We shall also be needing some well-known material concerning symmetric bilinear forms on $\mathbb{C}$-vector spaces. Let $S$ and $T$ be finite-dimensional $\mathbb{C}$-vector spaces equipped with nondegenerate symmetric bilinear forms $\langle-\mid-\rangle$. Nondegeneracy is the condition that, for all nonzero vectors $\sigma$ in $S$, the linear map $\langle\sigma \mid-\rangle: S \rightarrow \mathbb{C}$ is nonzero, or equivalently, $S$ has an orthonormal basis. Let $\mathbb{S}$ and $\mathbb{T}$ be orthonormal bases for $S$ and $T$, respectively. Let $\phi: T \rightarrow S$ be a $\mathbb{C}$-linear map. For $\sigma \in S$ and $\tau \in T$, we write $\langle\sigma|\phi| \tau\rangle=\langle\sigma \mid \phi(\tau)\rangle$. Thus, given $s \in \mathbb{S}$ and $t \in \mathbb{T}$, then $\langle s|\phi| t\rangle$ is the $(s, t)$-entry of the matrix representing $\phi$ with respect to the bases $\mathbb{S}$ and $\mathbb{T}$. Note that $\phi$ is an isometry if and only if, with respect to orthonormal bases, the matrices representing $\phi$ and $\phi^{-1}$ are the transposes of each other. That is to say, $\left\langle\phi(\tau) \mid \phi\left(\tau^{\prime}\right)\right\rangle=\left\langle\tau \mid \tau^{\prime}\right\rangle$ for all $\tau, \tau^{\prime} \in T$ if and only if $\langle s|\phi| t\rangle=\left\langle t\left|\phi^{-1}\right| s\right\rangle$ for all $s \in \mathbb{S}$ and $v \in \mathbb{T}$. 
In the previous section, we sketched Tornehave's construction of an $\mathbb{R} G$ module $W$, called a zombie module for an element $\kappa \in K(G)$ (with respect to $\alpha)$. Now we can give the full details. Consider, again, the element $\kappa=[X]-[Y]$. Regarding $\mathbb{R} X$ and $\mathbb{R} Y$ as inner product spaces with orthonormal bases $X$ and $Y$, we choose a $G$-invariant isometry $\imath: \mathbb{R} Y \rightarrow \mathbb{R} X$. Such an $\imath$ exists by the latest lemma. The inner products on $\mathbb{R} X$ and $\mathbb{R} Y$ extend $\mathbb{C}$-linearly to nondegenerate symmetric bilinear forms $\langle-\mid-\rangle$ on $\mathbb{C} X$ and $\mathbb{C} Y$. Moreover, $l$ extends $\mathbb{C}$-linearly to a $G$-invariant isometry $\imath: \mathbb{C} Y \rightarrow \mathbb{C} X$. We allow $\alpha$ to act on $\mathbb{C} X$ such that $\alpha\left(\sum_{x \in X} a_{x} x\right)=\sum_{x \in X} \alpha\left(a_{x}\right) x$, where each $a_{x} \in \mathbb{C}$. Similarly, we allow $\alpha$ to act on $\mathbb{C} Y$. As a function $\mathbb{C} X \rightarrow \mathbb{C} X$, we define

$$
\theta=l_{\circ} \alpha_{\circ} l^{-1} \alpha^{-1}
$$

We claim that $\theta$ is a $G$-invariant orthogonal $\mathbb{C}$-linear operator on $\mathbb{C} X$. (Our convention is to call a linear map an operator when its domain and codomain coincide.) The $G$-invariance of $\theta$ follows from the $G$-invariance of $l$ and $\alpha$. Straightforward manipulation yields

$$
\theta\left(\sum_{x \in X} a_{x} x\right)=\sum_{x^{\prime} \in X, y \in Y, x \in X}\left\langle x^{\prime}|\imath| y\right\rangle \alpha\left(\left\langle y\left|l^{-1}\right| x\right\rangle\right) a_{x} x^{\prime} .
$$

(The intermediate expressions are left to the reader.) So $\theta$ is $\mathbb{C}$-linear. Using the fact that $\langle x|\imath| y\rangle=\left\langle y\left|\imath^{-1}\right| x\right\rangle$ for all $x$ and $y$, we have

$$
\begin{aligned}
\left\langle x^{\prime}|\theta| x\right\rangle & =\sum_{y}\left\langle x^{\prime}|\imath| y\right\rangle \alpha\left(\left\langle y\left|l^{-1}\right| x\right\rangle\right) \\
& =\sum_{y} \alpha(\langle x|\imath| y\rangle)\left\langle y\left|l^{-1}\right| x^{\prime}\right\rangle=\left\langle x\left|\theta^{-1}\right| x^{\prime}\right\rangle .
\end{aligned}
$$

Therefore, $\theta$ is an orthogonal operator. The claim is now established.

A well-known theorem recorded in Bourbaki [11, III.6.10 (Rem. 1 of Def. 4)] asserts that, given a finite-dimensional real Lie group $\Gamma$ with complexification $\Gamma^{\mathbb{C}}$ and writing $\Gamma_{0}$ for the connected component of the identity element of $\Gamma$, then the embedding $\Gamma \hookrightarrow \Gamma^{\mathbb{C}}$ restricts to an identification $\left(\Gamma_{0}\right)^{\mathbb{C}}=\left(\Gamma^{\mathbb{C}}\right)_{0}$ and induces an isomorphism $\Gamma / \Gamma_{0} \cong \Gamma^{\mathbb{C}} / \Gamma_{0}^{\mathbb{C}}$. In other words, each element $\gamma \in \Gamma^{\mathbb{C}}$ can be deformed to an element $\gamma_{\mathbb{R}} \in \Gamma$ and, furthermore, the connected component of $\gamma_{\mathbb{R}}$ is well defined. As explained in, for instance, Onishchik-Vinberg [16, Section 5.1.3], the orthogonal group $O(\mathbb{C} X)$ is the complexification of the orthogonal group $O(\mathbb{R} X)$. It follows that $O_{G}(\mathbb{C} X)=O_{G}(\mathbb{R} X)^{\mathbb{C}}$ where $O_{G}$ indicates the group of $G$-invariant orthogonal operators. Therefore, $\theta$ can be deformed to a $G$-invariant orthogonal operator $\theta_{\mathbb{R}}$ on $\mathbb{R} X$, and the connected component of $\theta_{\mathbb{R}}$ is determined by $\theta$. We let $W$ be the $(-1)$ eigenspace of $\theta_{\mathbb{R}}$. Since $\theta$ is $G$-invariant, $W$ is an $\mathbb{R} G$-submodule of $\mathbb{R} X$.

The zombie module $W$ for $\kappa$ (with respect to $\alpha$ ) depends not only on $\kappa$ and $\alpha$ but also on the arbitrary choices that were made in the course of the construction: the choice of the pair of $G$-sets $X$ and $Y$; the choice of the $G$-invariant isometry $\imath$; the choice of the $G$-invariant real orthogonal operator $\theta_{\mathbb{R}}$. We call $\left(X, Y, l, \theta_{\mathbb{R}}\right)$ a choice tuple for $W$ as a zombie module for $\kappa$. Eventually, in Sections 6, 7, 8, we shall get to grips with the task of proving that the image of $[W]$ in $O_{\mathbb{R}}(G)$ is independent of the choice tuple. First, we need to take a closer look at the orientation functor $O_{\mathbb{R}}$. 


\section{THE ORIENTATION FUNCTOR}

Generally, for any subfield $\mathbb{K}$ of $\mathbb{C}$, we may consider the biset functor $A_{\mathbb{K}}$ associated with the $\mathbb{K} G$-character ring $A_{\mathbb{K}}(G)$, and we can define the quotient biset functor $O_{\mathbb{K}}=A_{\mathbb{K}} /\left(A_{\mathbb{K}} \cap I_{\mathbb{C}}\right)$, where $I_{\mathbb{C}}$ is the biset subfunctor of $A_{\mathbb{C}}$ whose coordinate module $I_{\mathbb{C}}(G)$ is spanned by those elements of $A_{\mathbb{C}}(G)$ that can be expressed as the sum $\chi+\chi^{\prime}$ of two Galois conjugate $\mathbb{C} G$-characters. Since $2 \chi \in$ $I_{\mathbb{C}}(G)$, we can regard $O_{\mathbb{K}}$ as a biset functor over $\mathbb{F}_{2}$. Via the evident isomorphism $O_{\mathbb{K}} \cong\left(A_{\mathbb{K}}+I_{\mathbb{C}}\right) / I_{\mathbb{C}}$, we have a chain of embeddings $O_{\mathbb{Q}} \leq O_{\mathbb{K}} \leq O_{\mathbb{C}}$.

Our concern, though, will be with the case $\mathbb{K}=\mathbb{R}$, which is of particular interest in connection with certain topological constructions. We have called $O_{\mathbb{R}}$ the orientation functor because, as we shall explain in this section, it can be used to record the orientation behaviour of certain kinds of $G$-homotopy automorphisms of certain kinds of $G$-spheres. At the end of this section, we shall give another interpretation of the zombie morphism $\mathrm{zom}^{\alpha}$ and the reduced Tornehave morphism $\overline{\text { torn }}^{\pi}$.

The reduced Tom Dieck map $\overline{\operatorname{die}}_{G}: A_{\mathbb{R}} \rightarrow B^{\times}(G)$ first arose in Tom Dieck's study [12, Sections $5.5,9.1,9.7]$ of $G$-homotopy maps between $G$-spheres. See also Tom Dieck [13, Sections II.8, III.2] and citations therein. In this context, the unit group $B^{\times}(G)$ plays two roles. Firstly, given a suitable $G$-space $X$, we can define the reduced Lefschetz invariant

$$
\tilde{\Lambda}(X)=\sum_{I \leq G} \tilde{\chi}\left(X^{I}\right) e_{I}^{G}
$$

as an element of $B(G)$. If each $I$-fixed subspace $X^{I}$ has the homotopy type of a sphere, then the reduced Euler characteristic $\tilde{\chi}\left(X^{I}\right)$ belongs to $\mathbb{Z}^{\times}$, hence $\widetilde{\Lambda}(X) \in$ $B^{\times}(G)$. Secondly, given a suitable $G$-map $\phi: X \rightarrow X$ for a suitable $G$-space $X$, we can define the reduced Lefschetz invariant

$$
\widetilde{\Lambda}(\phi)=\sum_{I \leq{ }_{G} G} \tilde{\Lambda}\left(\phi^{I}\right) e_{I}^{G}
$$

in $B(G)$, where $\widetilde{\Lambda}\left(\phi^{I}\right)$ is the reduced Lefschetz number of the restriction of $\phi$ to a map $\phi_{\sim}^{I}: X^{I} \rightarrow X^{I}$. We mean to say that, as a sum with only finitely many nonzero terms, $\tilde{\Lambda}\left(\phi^{I}\right)=\sum_{n=-1}^{\infty}(-1)^{n} \operatorname{tr}\left(\phi_{n}^{I}\right)$, where the traces, here, are the traces of the maps $\phi_{n}^{I} \in \operatorname{End}_{\mathbb{Z}}\left(\widetilde{H}_{n}\left(X^{I}\right)\right)$ induced on the reduced homology groups $\widetilde{H}_{n}\left(X^{I}\right)$. If $X$ is a $G$ homotopy sphere and $\phi$ has a $G$-homotopy inverse then, again, $\widetilde{\Lambda}(\phi) \in B^{\times}(G)$. But, for such $X$ and $\phi$, the reduced Lefschetz invariant can usefully be replaced by the degree invariant

$$
\operatorname{deg}(\phi)=\sum_{I \leq{ }_{G} G} \operatorname{deg}\left(\phi^{I}\right) e_{I}^{G}
$$

where $\operatorname{deg}\left(\phi^{I}\right)$ is the degree of $\phi^{I}$. That is to say, if $X^{I}$ has the homotopy type of an $m$-sphere, for some integer $m \geq-1$, then $\phi^{I}$ acts on the unique nonzero reduced homology group $\widetilde{H}_{m}\left(X^{I}\right) \cong \mathbb{Z}$ as multiplication by the integer $\operatorname{deg}\left(\phi^{I}\right)= \pm 1$. It is to be understood that, if $X^{I}=\emptyset$, then $m=-1$ and $\operatorname{deg}\left(\phi^{I}\right)=1$. It is not hard to show 
that the degree invariant and the reduced Lefschetz invariant are related by

$$
\operatorname{deg}(\phi)=-\tilde{\Lambda}\left(\delta_{\circ} \phi\right)=-\tilde{\Lambda}\left(\phi_{\circ} \delta\right),
$$

where $\delta$ is the antipodal $G$-map on $X$. It follows that $\operatorname{deg}(\phi) \in B^{\times}(G)$. One advantage of the degree invariant is that, unlike the Lefschetz invariant, it has the multiplicative property $\operatorname{deg}\left(\phi_{\circ} \phi^{\prime}\right)=\operatorname{deg}(\phi) \cdot \operatorname{deg}\left(\phi^{\prime}\right)$.

For the rest of this section, we shall confine our discussion to the linear case: the homotopy $G$-spheres will be associated with $\mathbb{R} G$-modules, and the homotopy $G$-automorphisms will be associated with $\mathbb{R} G$-automorphisms of $\mathbb{R} G$-modules. Our account will be self-contained, without making appeals to the more general theory indicated above. Besides, appeals to the general theory would not help very much, since most of the difficulty in our discussion will be in showing how, in the linear case, the orientation group $O_{\mathbb{R}}(G)$ can serve as a refinement of the unit group $B^{\times}(G)$.

Remark 4.1. Writing ori : $A_{\mathbb{R}} \rightarrow O_{\mathbb{R}}$ for the canonical epimorphism, the set $\left\{\operatorname{ori}_{G}(\chi): \chi \in_{\mathrm{Gal}} \operatorname{AbsIrr}(\mathbb{R} G)\right\}$ is an $\mathbb{F}_{2}$-basis for $O_{\mathbb{R}}(G)$, where the notation indicates that $\chi$ runs over representatives of the Galois conjugacy classes in the set $\operatorname{Abs} \operatorname{Irr}(\mathbb{R} G)$ of absolutely irreducible $\mathbb{R} G$-characters.

Proof. The set of irreducible $\mathbb{C} G$-characters $\operatorname{Irr}(\mathbb{C} G)$ is a $\mathbb{Z}$-basis for $A_{\mathbb{C}}(G)$, so $\left\{\chi+I_{\mathbb{C}}(G): \chi \in_{\mathrm{Gal}} \operatorname{Irr}(\mathbb{C} G)\right\}$ is an $\mathbb{F}_{2}$-basis for $A_{\mathbb{C}}(G) / I_{\mathbb{C}}(G)$. Therefore, $\operatorname{ori}_{G}(\chi)$ : $\left.\chi \in \in_{\mathrm{Gal}} \operatorname{AbsIrr}(\mathbb{R} G)\right\}$ is linearly independent. On the other hand, the set of irreducible $\mathbb{R} G$-characters $\operatorname{Irr}(\mathbb{R} G)$ is a $\mathbb{Z}$-basis for $A_{\mathbb{R}}(G)$, so $\left\{\operatorname{ori}_{G}(\chi): \chi \in_{\mathrm{Gal}} \operatorname{Irr}(\mathbb{R} G)\right\}$ spans $O_{\mathbb{R}}(G)$. If $\chi$ is not absolutely irreducible, then $\chi$ is the sum of two Galois conjugate $\mathbb{C} G$-characters, hence ori ${ }_{G}(\chi)=0$. Therefore, $\left\{\operatorname{ori}_{G}(\chi): \chi \in_{\mathrm{Gal}} \operatorname{AbsIrr}(\mathbb{R} G)\right\}$ spans $O_{\mathbb{R}}(G)$.

Let us introduce a notation for expressing coordinates with respect to the $\mathbb{F}_{2^{-}}$ basis specified in the remark. Given an element $\omega \in O_{\mathbb{R}}(G)$, we write

$$
\omega=\sum_{\chi \in \in_{\text {Gal }}} \operatorname{AbsIrr}(\mathbb{R} G)[\chi @ \omega] \operatorname{ori}_{G}(\chi),
$$

where each $[\chi @ \omega] \in \mathbb{F}_{2}$. Recall that the set of irreducible $\mathbb{C} G$-characters is an orthonormal basis for the usual inner product $\langle-\mid-\rangle_{G}$ on the $\mathbb{C}$-vector space $\mathbb{C} A_{\mathbb{C}}(G)$. The multiplicity of an absolutely irreducible $\mathbb{R} G$-character $\chi$ in a given element $\rho \in A_{\mathbb{R}}(G)$ is $\langle\chi \mid \rho\rangle_{G}$. Therefore,

$$
\operatorname{ori}_{G}(\rho)=\sum_{\chi \in \operatorname{AbsIrr}(\mathbb{R} G)}\langle\chi \mid \rho\rangle_{G} \operatorname{ori}_{G}(\chi) .
$$

So the $\chi$ th coordinate of $\operatorname{ori}_{G}(\rho)$ is

$$
\left[\chi @ \operatorname{ori}_{G}(\rho)\right] \stackrel{\text { par }}{=} \sum_{\chi^{\prime} \in[\chi]_{\text {Gal }}}\left\langle\chi^{\prime} \mid \rho\right\rangle_{G}
$$

as a sum in $\mathbb{F}_{2}$, where $[\chi]_{\text {Gal }}$ denotes the set of Galois conjugates of $\chi$. 
Plainly, the diagram depicted on the left, below, is a commutative diagram of morphisms of biset functors. Some more notation will be needed to explain the diagram on the right, a commutative triangle of group homomorphisms. Consider an $\mathbb{R} G$-module $M$ equipped with a $G$-invariant inner product. We shall define the degree homomorphism $\operatorname{deg}_{M}$ and the orientation homomorphism ori ${ }_{M}$ as functions, we shall check the commutativity of the triangle, then we shall prove that $\operatorname{deg}_{M}$ and ori $_{M}$ are homomorphisms.
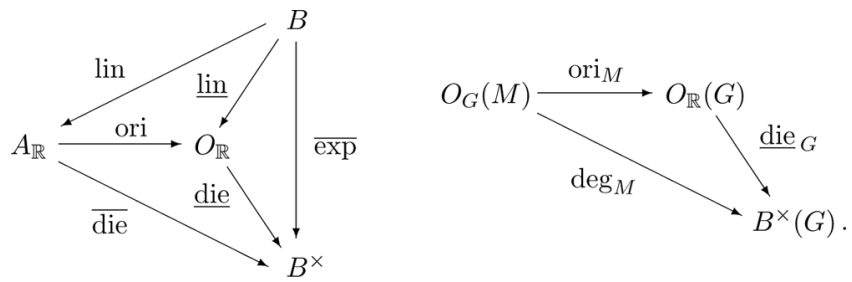

Let $O_{G}(M)$ denote the group of $G$-invariant orthogonal operators on $M$. For any $\phi \in O_{G}(M)$, the $(-1)$-eigenspace of $\phi$, denoted $W_{\phi}$, is an $\mathbb{R} G$-submodule of $M$. We define

$$
\operatorname{deg}_{M}(\phi)=\overline{\operatorname{die}}_{G}\left[W_{\phi}\right], \quad \operatorname{ori}_{M}(\phi)=\operatorname{ori}_{G}\left[W_{\phi}\right]
$$

Plainly, we have a commutative triangle of functions $\operatorname{deg}_{M}=\overline{\operatorname{die}}_{G \circ}$ ori ${ }_{M}$. To prove the group homomorphism property of $\operatorname{deg}_{M}$ and ori ${ }_{M}$, some work will be needed. Let us begin this by making an observation in a context where no $G$-actions are involved.

Remark 4.2. Consider an orthogonal operator $\psi$ on a finite-dimensional real inner product space $L$. Let $W_{\psi}$ denote the $(-1)$-eigenspace of $\psi$. Let $S(L)$ denote the unit sphere of $L$, and let $\operatorname{deg}(\psi)$ denote the degree of $\psi$ as a homeomorphism $S(L) \rightarrow$ $S(L)$. Then

$$
\operatorname{par}\left(\operatorname{dim}_{\mathbb{R}}\left(W_{\psi}\right)\right)=\operatorname{det}(\psi)=\operatorname{deg}(\psi) .
$$

Proof. Let $\psi_{\mathbb{C}}$ be the $\mathbb{C}$-linear extension of $\psi$ to an operator on the complex vector space $\mathbb{C} L$. The integer $\operatorname{dim}_{\mathbb{R}}\left(W_{\psi}\right)$ is the multiplicity of -1 as an eigenvalue of $\psi$, and it is also the multiplicity of -1 as an eigenvalue of $\psi_{\mathbb{C}}$. The only other possible real eigenvalue of $\psi_{\mathbb{C}}$ is 1 . If $\lambda$ is a nonreal eigenvalue of $\psi_{\mathbb{C}}$, then $|\lambda|=$ 1 and the complex conjugate $\lambda^{*}$ is an eigenvalue with the same multiplicity as $\lambda$. So $\operatorname{par}\left(\operatorname{dim}_{\mathbb{R}}\left(W_{\psi}\right)\right)=\operatorname{det}\left(\psi_{\mathbb{C}}\right)=\operatorname{det}(\psi)$. If $\psi$ preserves the orientation of $S(L)$, then $\operatorname{det}(\psi)=1=\operatorname{deg}(\psi)$ otherwise, $\operatorname{det}(\psi)=-1=\operatorname{deg}(\psi)$.

The next remark is essentially the same observation but more conveniently expressed.

Remark 4.3. Let $M, \phi, W_{\phi}$ be as above. Let $U$ be an $\operatorname{End}_{\mathbb{R} G}(M)$-submodule of $M$. Then $\phi$ acts as an orthogonal operator on $U$. Write $\operatorname{det}(\phi: U)$ for the determinant of $\phi$ acting on $U$, and write $\operatorname{deg}(\phi: S(U))$ for the degree of $\phi$ acting on $S(U)$. Then

$$
\operatorname{par}\left(\operatorname{dim}_{\mathbb{R}}\left(W_{\phi} \cap U\right)\right)=\operatorname{det}(\phi: U)=\operatorname{deg}(\phi: S(U))
$$

and, moreover, this element of $\mathbb{Z}^{\times}$depends only on the isomorphism class of $U$. 
Proof. We have $\phi \in \operatorname{End}_{\mathbb{R} G}(M)$, so $\phi$ acts on $U$ and, moreover, $\operatorname{det}(\phi: U)$ depends only on the isomorphism class of $U$. Obviously, $\phi$ acts as an orthogonal operator on $U$. The rest follows from the previous remark by putting $L=U$.

Proposition 4.4. With the notation of the latest remark, the coordinate of $\operatorname{deg}_{M}(\phi)$ at a subgroup $I \leq G$ is $\left[\operatorname{deg}_{M}(\phi) @ I\right]=\operatorname{dim}_{\mathbb{R}}\left(W_{\phi}^{I}\right)$ as an element of $\mathbb{F}_{2}$. That is to say, in the multiplicative notation, the element $\operatorname{deg}_{M}(\phi) \in B^{\times}(G)$ is given by

$$
\epsilon_{I}^{G}\left(\operatorname{deg}_{M}(\phi)\right)=\operatorname{par}\left[\operatorname{deg}_{M}(\phi) @ I\right]=\operatorname{det}\left(\phi: M^{I}\right)=\operatorname{deg}\left(\phi\left(S\left(M^{I}\right)\right)\right) .
$$

In particular, $\operatorname{deg}_{M}: O_{G}(M) \rightarrow B^{\times}(G)$ is a group homomorphism.

Proof. The definitions of $\operatorname{deg}_{\phi}$ and $\operatorname{die}_{G}$ yield $\left[\operatorname{deg}_{M}(\phi) @ I\right]=\operatorname{dim}_{\mathbb{R}}\left(W_{\phi}^{I}\right)$ as an element of $\mathbb{F}_{2}$. Putting $U=M^{I}$, the required equality holds by the remark. The rider follows from the multiplicative property of determinants or, alternatively, from the multiplicative property of degrees.

To deal with ori ${ }_{G}$, we need some further notation. Consider an absolutely irreducible $\mathbb{R} G$-character $\chi$. Letting $e_{\chi}$ be the primitive idempotent of $Z(\mathbb{R} G)$ associated with $\chi$, then

$$
e_{\chi} M \cong U_{\chi} \otimes_{\mathbb{R}} S_{\chi}
$$

as an isomorphism of modules of the algebra $\operatorname{End}_{\mathbb{R} G}(M) \otimes_{\mathbb{R}} \mathbb{R} G$, where $U_{\gamma}$ is a simple $\operatorname{End}_{\mathbb{R} G}(M)$-module and $S_{\chi}$ is the simple $\mathbb{R} G$-module with character $\chi$. Of course, the simple modules $U_{\chi}$ and $S_{\chi}$ are unique up to isomorphism. We call $U_{\chi}$ the simple $\operatorname{End}_{\mathbb{R} G}(M)$-module associated with $\chi$. If $\chi$ does not occur in $M$, we understand that $U_{\chi}$ is the zero module and, in that case, the unique operator on $U_{\chi}$ is understood to have determinant 1 and the unique map on the unit sphere $S\left(U_{\chi}\right)$ is understood to have degree 1 . Note that the multiplicity of $S_{\chi}$ in $M$ is $\langle\chi \mid[M]\rangle_{G}=$ $\operatorname{dim}_{\mathbb{R}}\left(U_{\chi}\right)$.

Lemma 4.5. With the notation above, the multiplicity $\left\langle\chi \mid W_{\phi}\right\rangle_{G}$ of $\chi$ in the (-1)eigenspace $W_{\phi}$ is given, up to parity, by

$$
\operatorname{par}\left\langle\chi \mid W_{\phi}\right\rangle_{G}=\operatorname{det}\left(\phi: U_{\chi}\right)=\operatorname{deg}\left(\phi: S\left(U_{\chi}\right)\right) .
$$

Proof. We may assume that $S_{\chi}$ occurs in $M$, because otherwise all three expressions in the specified equation have value 1. Although the simple module $U_{\chi}$ is defined only up to isomorphism, the rider of Remark 4.3 tells us that $\operatorname{det}\left(\phi: U_{\gamma}\right)$ and $\operatorname{deg}(\phi$ : $\left.S\left(U_{\gamma}\right)\right)$ are well defined and equal to each other.

We shall construct an $\operatorname{End}_{\mathbb{R} G}(M)$-submodule $\widehat{U}_{\chi} \leq e_{\chi} M$ such that $\widehat{U}_{\gamma} \cong U_{\gamma}$. Let $m=\langle\chi \mid[M]\rangle_{G}=\operatorname{dim}_{\mathbb{R}}\left(U_{\chi}\right)$. Write $e_{\chi} M=S_{1} \oplus \cdots \oplus S_{m}$ as a direct sum of mutually orthogonal $\mathbb{R} G$-modules isomorphic to $S_{\chi}$. Choose a vector $u \in S_{1}$. For each $1 \leq$ $j \leq m$, we have $\operatorname{Hom}_{\mathbb{R} G}\left(S_{1}, S_{j}\right) \cong \mathbb{R}$, so there exists a unit vector $u_{j} \in S_{j}$ (unique up to a factor of \pm 1$)$ such that $\operatorname{Hom}_{\mathbb{R} G}\left(S_{1}, S_{j}\right) . \mathbb{R} u=\mathbb{R} u_{j}$. The set $\left\{u_{1}, \ldots, u_{m}\right\}$ is an orthonormal basis for a simple $\operatorname{End}_{\mathbb{R} G}(M)$-submodule $\widehat{U}_{\chi}$ of $e_{\chi} M$. But $e_{\chi} M$ is a direct sum of copies of $U_{\chi}$, so $\widehat{U}_{\chi} \cong U_{\chi}$. 
As we noted in Remark 4.3, $\phi$ acts as an orthogonal operator $\widehat{\phi}$ on $\widehat{U}_{\chi}$. Extending $\mathbb{C}$-linearly, $e_{\chi} \mathbb{C} M \cong \mathbb{C} \widehat{U}_{\chi} \otimes_{\mathbb{C}} \mathbb{C} S_{\chi}$ as modules of $\operatorname{End}_{\mathbb{C} G}(\mathbb{C} M)$. So the action of $\phi$ on $e_{\chi} \mathbb{C} M$ and the action of $\widehat{\phi}$ on $\mathbb{C} \widehat{U}_{\chi}$ have the same eigenvalues. For each eigenvalue $\lambda$, let $E_{\lambda} \leq e_{\chi} \mathbb{C} M$ and $\widehat{E}_{\lambda} \leq \mathbb{C} \widehat{U}_{\gamma}$ be the corresponding eigenspaces. Observing that $e_{\chi} \mathbb{C} M$ and $\mathbb{C} \widehat{U}_{\chi}$ are the direct sums of the eigenspaces, it is easy to show that $E_{\lambda}$ is the $\mathbb{R} G$-submodule generated by $\widehat{E}_{\lambda}$ and

$$
\operatorname{dim}_{\mathbb{C}}\left(E_{\lambda}\right)=\operatorname{dim}_{\mathbb{C}}\left(\widehat{E}_{\lambda}\right) \cdot \operatorname{dim}_{\mathbb{C}}\left(\mathbb{C} S_{\chi}\right)
$$

Complexification of a real operator does not change the dimension of an eigenspace associated with a real eigenvalue. So, putting $\lambda=-1$ and noting that the $(-1)$ eigenspace of the action of $\phi$ on $\widehat{U}_{\chi}$ is $W_{\phi} \cap \widehat{U}_{\chi}$, we deduce that the $(-1)$-eigenspace of $\phi$ on $e_{\chi} M$ is the direct sum of $\operatorname{dim}_{\mathbb{R}}\left(W_{\phi} \cap \widehat{U}_{\chi}\right)$ copies of $S_{\chi}$. In other words, $\left\langle\chi \mid\left[W_{\phi}\right]\right\rangle_{G}=\operatorname{dim}_{\mathbb{R}}\left(W_{\phi} \cap \widehat{U}_{\chi}\right)$. By Remark 4.3, $\operatorname{par}\left\langle\chi \mid W_{\phi}\right\rangle_{G}=\operatorname{det}\left(\phi: \widehat{U}_{\chi}\right)=\operatorname{deg}(\phi:$ $\left.S\left(\widehat{U}_{\chi}\right)\right)$.

Proposition 4.6. With the notation above, and defining $U_{\chi}^{\mathrm{Gal}}=\bigoplus_{\chi^{\prime} \in[\chi]_{\mathrm{Gal}}} U_{\chi^{\prime}}$, the $\chi$ th coordinate $\left[\chi @ \operatorname{ori}_{M}(\phi)\right]$ of ori $_{M}(\phi)$ is given by

$$
\operatorname{par}\left[\chi @ \operatorname{ori}_{M}(\phi)\right]=\operatorname{det}\left(\phi: U_{\chi}^{\mathrm{Gal}}\right)=\operatorname{deg}\left(\phi: S\left(U_{\chi}^{\mathrm{Gal}}\right)\right) .
$$

In particular, ori $_{M}: O_{G}(M) \rightarrow O_{\mathbb{R}}(G)$ is a group homomorphism.

Proof. Using the latest lemma and a formula above for the $\chi$ th coordinate,

$$
\operatorname{par}\left[\chi @ \operatorname{ori}_{M}(\phi)\right]=\operatorname{par}\left[\chi @ \operatorname{ori}_{G}\left(W_{\phi}\right)\right]=\prod_{\chi^{\prime}} \operatorname{det}\left(\phi: U_{\chi^{\prime}}\right)=\prod_{\chi^{\prime}} \operatorname{deg}\left(\phi: S\left(U_{\chi^{\prime}}\right)\right) .
$$

The rider follows easily, as in the proof of Proposition 4.4.

The commutative triangle of group homomorphisms depicted above has now been established. The two propositions in this section also give formulas for $\operatorname{deg}_{M}(\phi)$ and $\operatorname{ori}_{M}(\phi)$. The formula for $\operatorname{deg}_{M}(\phi)$ will be used in Section 7 to prove Proposition 2.2. The formula for $\operatorname{ori}_{G}(\phi)$ will be used in Section 8 to prove Theorem 2.3. Those two applications will also make use of the following obvious remark.

Remark 4.7. Given an element $\kappa \in K(G)$, let $\theta_{\mathbb{R}}$ be the element of $O_{G}(\mathbb{R} X)$ constructed from $\kappa$ as in Section 3, and let $W$ be the (-1)-eigenspace of $\theta_{\mathbb{R}}$. Then $\operatorname{ori}_{G}[W]=\operatorname{ori}_{\mathbb{R} X}\left(\theta_{\mathbb{R}}\right)$.

The next remark, again obvious, points out a relationship between the two commutative triangles $\overline{\operatorname{die}}_{G}=\underline{\operatorname{die}}_{G \circ}$ ori ${ }_{G}$ and $\operatorname{deg}_{M}=\underline{\operatorname{die}}_{G \circ}$ ori ${ }_{M}$.

Remark 4.8. For $M$ as above, $\overline{\operatorname{die}}_{G}[M]=\operatorname{deg}_{M}\left(-\mathrm{id}_{M}\right)$ and $\operatorname{ori}_{G}[M]=\operatorname{ori}_{M}\left(-\mathrm{id}_{M}\right)$.

Taking $M$ to be a permutation $\mathbb{R} G$-module, we obtain the following description of the exponential map $\overline{\exp }_{G}$ and the condensed linearization map $\overline{\operatorname{lin}}_{G}$. 
Remark 4.9. Given a $G$-set $X$, then $\overline{\exp }_{G}[X]=\operatorname{deg}_{\mathbb{R} X}\left(-\mathrm{id}_{\mathbb{R} X}\right)$ and $\overline{\operatorname{lin}}_{G}[X]=$ ori $_{\mathbb{R} X}\left(-\mathrm{id}_{\mathbb{R} X}\right)$.

We noted Remark 4.9, banal as it is, because there is a similar description of the zombie map zom ${ }_{G}^{\alpha}$ and the reduced Tornehave map $\overline{\operatorname{torn}}_{G}^{\pi}$. Indeed, when we have proved Theorem 2.3, we shall immediately obtain the following more satisfying rendition of Remark 4.7.

Corollary 4.10. Given $\kappa \in K(G)$ and letting $\theta_{\mathbb{R}}$ be as above, $\overline{\operatorname{torn}}_{G}^{\pi}(\kappa)=\operatorname{deg}_{\mathbb{R} X}\left(\theta_{\mathbb{R}}\right)$ and $\operatorname{zom}_{G}^{\alpha}(\kappa)=\operatorname{ori}_{\mathbb{R} X}\left(\theta_{\mathbb{R}}\right)$.

Let us mention that our use of inner products is not crucial to the commutative triangle of group homomorphisms $\operatorname{deg}_{M}=\underline{\operatorname{die}}_{G \circ}$ ori ${ }_{M}$. If we drop the assumption that $M$ is equipped with a $G$-invariant inner product, then we can still construct a commutative triangle with $O_{G}(M)$ replaced by the group of $G$-invariant linear automorphisms $\mathrm{GL}_{G}(M)$. In place of the unit sphere $S(M)$, we can consider the punctured space $M-\{0\}$ or the one-point compactification $M \cup\{\infty\}$, both of which are $G$-homotopy spheres. The use of $(-1)$-eigenspaces cannot be adapted to this context, but the group homomorphism properties of $\operatorname{deg}_{M}$ and ori $_{M}$ can still be established using degrees or signs of determinants. One extra difficulty that does arise is in showing that the image of the generalized degree map $\operatorname{deg}_{M}: \mathrm{GL}_{G}(M) \rightarrow$ $\beta^{\times}(G)$ is contained in $B^{\times}(G)$, but hints on a proof can be found in Tom Dieck [13, Exercise II.10.28.7]. Alternatively, we can impose an arbitrarily chosen $G$-invariant inner product on $M$ and then deform operators in $\mathrm{GL}_{G}(M)$ to operators in $O_{G}(M)$. Our reason for not working in this more general context is that, in our applications below, $G$-invariant inner products arise naturally, and it will be convenient to make use of them.

\section{FOR NILPOTENT GROUPS}

For nilpotent groups, some of the material in the previous section descends into triviality by Theorem 2.1, which we are about to prove. We shall be needing the following theorem of Bouc, essentially $[8,8.5,9.5,9.6]$. which is recorded in $[1$, $6.6]$.

Theorem 5.1 (Bouc). If $G$ is nilpotent, then $\operatorname{dim}_{\mathbb{F}_{2}}\left(B^{\times}(G)\right)$ is equal to the number of Galois conjugacy classes of absolutely irreducible $\mathbb{R} G$-characters.

Proof. In the case where $G$ is a 2-group, this version of Bouc's result appears in $[1,6.6]$. For arbitrary nilpotent $G$, let us write $G=P \times P^{\prime}$ where $P$ is the Sylow 2-subgroup and $P^{\prime}$ is the Hall $2^{\prime}$-subgroup. Bouc [8, 6.3] observed that the map $\inf _{G, G / P^{\prime} \circ}$ iso $_{G / P^{\prime}, P}: B^{\times}(P) \rightarrow B^{\times}(G)$ is an $\mathbb{F}_{2}$-linear isomorphism. On the other hand, the map $\inf _{G, G / P^{\prime} \circ}$ iso $_{G / P^{\prime}, P}: A_{\mathbb{R}}(P) \rightarrow A_{\mathbb{R}}(G)$ provides a bijection $\operatorname{AbsIrr}(\mathbb{R} P) \rightarrow \operatorname{Abs} \operatorname{Irr}(\mathbb{R} G)$, and this bijection is preserved under the action of Galois automorphisms. Thus we have reduced to the case where $G$ is a 2-group.

To complete the proof of Theorem 2.1, we also need the following result of Tornehave [17]. Some comments on proofs of this result were made in Section 2. 
Again, it was originally stated only in the case where $G$ is a 2-group, but the general nilpotent case follows immediately from the surjectivity of the map $\inf _{G, G / P^{\prime} \text { o }}$ iso $_{G / P^{\prime}, P}: B^{\times}(P) \rightarrow B^{\times}(G)$.

Theorem 5.2 (Tornehave). If $G$ is nilpotent, then the reduced Tom Dieck map $\overline{\operatorname{die}}_{G}$ : $A_{\mathbb{R}}(G) \rightarrow B^{\times}(G)$ is surjective.

Theorem 2.1 now follows because, for nilpotent $G$, Theorem 5.2 implies that the condensed Tom Dieck morphism $\overline{\operatorname{die}}_{G}: O_{\mathbb{R}}(G) \rightarrow B^{\times}(G)$ is surjective, while Theorem 5.1 implies that $\operatorname{dim}_{\mathbb{F}_{2}}\left(O_{\mathbb{R}}(G)\right)=\operatorname{dim}_{\mathbb{F}_{2}}\left(B^{\times}(G)\right)$. The conclusions of all three theorems fail if we drop the hypothesis that $G$ is nilpotent.

Remark 5.3. Putting $G=A_{5}$, $\underline{\text { die }}_{G}$ is not surjective. Putting $G=\mathrm{SD}_{16} \ltimes C_{3}$ as a semidirect product, where the conjugation action of $\mathrm{SD}_{16}$ on $C_{3}$ has kernel isomorphic to $Q_{8}$, then $\underline{\mathrm{die}}_{G}$ is not injective.

Proof. Suppose that $G=A_{5}$. On the set of subgroups of $G$, we introduce an equivalence relation $\equiv$ whose five equivalence classes are $\left\{1, C_{2}, C_{3}, C_{5}\right\},\left\{V_{4}, A_{4}\right\}$, $\left\{S_{3}\right\},\left\{D_{10}\right\},\left\{A_{5}\right\}$. We mean to say that the cyclic subgroups of $G$ comprise one equivalence class, the subgroups isomorphic to $V_{4}$ or $A_{4}$ comprise another equivalence class, and so on. Yoshida's Criterion $[19,6.5]$ is a necessary and sufficient criterion for a given unit of the ghost ring to belong to $B^{\times}(G)$. Using Yoshida's Criterion, is it is easy to show that an element $x \in \beta^{\times}(G)$ belongs to $B^{\times}(G)$ if and only if $[x @ I]=\left[x @ I^{\prime}\right]$ whenever $I \equiv I^{\prime}$. In particular, $\operatorname{dim}_{\mathbb{F}_{2}}\left(B^{\times}(G)\right)=5$. On the other hand, there are precisely 4 Galois conjugacy classes of absolutely irreducible $\mathbb{R} G$-characters, so $\operatorname{dim}\left(O_{\mathbb{R}}(G)\right)=4$. Therefore, $\underline{\operatorname{die}}_{G}$ cannot be surjective. (As the referee has pointed out, several other counterexamples to surjectivity can be established using the dimensions of Burnside unit groups recorded in Boltje-Pfeiffer [5, Section 4].)

For the semidihedral group $\mathrm{SD}_{16}$ with order 16, the three maximal subgroups are isomorphic to $Q_{8}, D_{8}, C_{8}$. So there exists a unique semidirect product $G=$ $\mathrm{SD}_{16} \ltimes C_{3}$ such that the kernel of the conjugation action of $\mathrm{SD}_{16}$ on $C_{3}$ is isomorphic to $Q_{8}$. We shall show that there exists a unique faithful irreducible $\mathbb{R} G$-character $\chi$ moreover, $\chi$ is absolutely irreducible and $\overline{\operatorname{die}}_{G}(\chi)=0$. It will then follow that $\mathrm{die}_{G}$ annihilates the nonzero element ori ${ }_{G}(\chi)$ of $O_{\mathbb{R}}(G)$, and hence $\underline{\text { die }}_{G}$ is not injective.

We write $G=S \ltimes C$, where $S=\left\langle a, s: a^{8}=s^{2}=1\right.$, sas $\left.s^{-1}=a^{3}\right\rangle \cong \mathrm{SD}_{16}$ and $C=\left\langle v: v^{3}=1\right\rangle \cong C_{3}$, with $S$ acting on $C$ such that $a v a^{-1}=s v s^{-1}=v^{2}$. The centralizer $Q=C_{S}(C)$ consists of the elements having the form $a^{n}$ and $s a^{m}$ where $n$ is even and $m$ is odd. Six of the eight elements of $Q$ have order 4 , so $Q \cong Q_{8}$. A Clifford-theoretic argument shows that any faithful irreducible $\mathbb{C} G$-character $\chi$ is induced from one of the two faithful irreducible $\mathbb{C}(Q \times C)$-characters. By direct calculation, such $\chi$ is unique, and it has the values shown in the table below. For each representative $g$ of the conjugacy classes in $G$, the table shows the order $|\langle g\rangle|$ of $g$ and the size $|[g]|$ of the conjugacy class $[g]$. The table also records the values of $g^{2}$, which are used to show that the Frobenius-Schur indicator of $\chi$ is 1 ; hence, $\chi$ is an absolutely irreducible $\mathbb{R} G$-character. 


\begin{tabular}{r|cc|cccc|cc|ccc}
$g^{2}$ & 1 & $v^{2}$ & 1 & $v^{2}$ & $a^{4}$ & $a^{4} v^{2}$ & $a^{2}$ & $a^{6}$ & 1 & $a^{4}$ & $a^{4} v^{2}$ \\
$|[g]|$ & 1 & 2 & 1 & 2 & 2 & 4 & 6 & 6 & 12 & 4 & 8 \\
$|\langle g\rangle|$ & 1 & 3 & 2 & 6 & 4 & 12 & 8 & 8 & 2 & 4 & 12 \\
$g$ & 1 & $v$ & $a^{4}$ & $a^{4} v$ & $a^{2}$ & $a^{2} v$ & $a$ & $a^{7}$ & $s$ & $s a$ & $s a v$ \\
\hline$\chi$ & 4 & -2 & -4 & 2 & 0 & 0 & 0 & 0 & 0 & 0 & 0
\end{tabular}

Let $S_{\chi}$ be a simple $\mathbb{R} G$-module affording $\chi$. For a contradiction, suppose there exists a subgroup $I$ of $G$ such that the $I$ th coordinate $\left[\overline{\operatorname{die}}_{G}(\chi) @ I\right]$ is nonzero. In other words, the integer $\operatorname{dim}_{\mathbb{R}}\left(S_{\chi}^{I}\right)=\left\langle 1 \mid \operatorname{res}_{I, G}(\chi)\right\rangle_{I}$ is odd. Since $\operatorname{dim}_{\mathbb{R}}\left(S_{\chi}^{C}\right)=$ $\left\langle 1 \mid \operatorname{res}_{C, G}\right\rangle_{C}=0$, we have $I \cap C=1$. So $I$ is a 2-subgroup. Replacing $I$ with a suitable $G$-conjugate, we may assume that $I \leq S$. A similar argument shows that $I \cap\left\langle a^{4}\right\rangle=$ 1. But $\left\langle a^{4}\right\rangle$ is the unique minimal nontrivial subgroup of $Q$, so $I \cap Q=1$. Therefore, $I=1$ or $I$ is $P$-conjugate to $\langle s\rangle$. But, in those two cases, $\operatorname{dim}_{\mathbb{R}}\left(S_{\chi}^{I}\right)$ is 0 or 2 , respectively. Neither of those two dimensions being odd, we have a contradiction, as required.

Let us mention a remarkable curiosity. Few finite groups $G$ have the property that, for some equivalence relation $\equiv$ on the set of subgroups of $G$, an element $x \in$ $\beta^{\times}(G)$ belongs to $B^{\times}(G)$ if and only if $[x @ I]=\left[x @ I^{\prime}\right]$ whenever $I \equiv I^{\prime}$. Above, we made use of the fact that such an equivalence relation $\equiv$ does exist in the case of the group $A_{5}=\mathrm{PSL}_{2}(5)$. Using Yoshida's Criterion and results on group structure in Huppert [15, II.8], it can be shown that, in fact, the group $\operatorname{PSL}_{2}(p)$ has this property for any prime $p$. Let us omit the proof, which is several pages long.

\section{DETERMINANTS}

Tornehave proved Proposition 2.2 by considering determinants of certain orthogonal operators. Theorem 2.3 yields to the same method, but the necessary preliminary results are more general and more intricate. In this section, we shall establish those preliminaries in the generality that will be needed for the latter application. In the next section, we shall recover Proposition 2.2 and, in the section after that, we shall complete the proof of Theorem 2.3.

Our heavy use of matrices seems to be unavoidable. A major difficulty in examining the $\mathbb{C}$-linear map $\theta=l_{0} \alpha_{0} l^{-1}{ }_{\circ} \alpha^{-1}$ is that the functions $\alpha: \mathbb{C} Y \rightarrow \mathbb{C} Y$ and $\alpha^{-1}: \mathbb{C} X \rightarrow \mathbb{C} X$ are not $\mathbb{C}$-linear. To find the determinants of certain restrictions of $\theta$, we shall replace $\alpha$ and $\alpha^{-1}$ with suitable $\mathbb{C}$-linear maps $v$ and $\mu$ defined by formulas for their matrix entries.

Let us return to the scenario that we discussed at the end of Section 3. Fixing an element $\kappa \in K(G)$, let $\left(X, Y, \imath, \theta_{\mathbb{R}}\right)$ be a choice tuple for a zombie module $W$ for $\kappa$ (with respect to $\alpha$ ). Recall that $W$ is the $(-1)$-eigenspace of the element $\theta_{\mathbb{R}} \in$ $O_{G}(\mathbb{R} X)$. Also recall that $\theta_{\mathbb{R}}$ is a deformation of the element $\theta \in O_{G}(\mathbb{C} X)$ where $\theta=l_{\circ} \alpha_{\circ} l^{-1}{ }_{\circ} \alpha^{-1}$. Writing $\mathscr{E}=\operatorname{End}_{\mathbb{R} G}(\mathbb{R} X)$, we now consider an $\mathscr{E}$-submodule $U$ of $\mathbb{R} X$. We have $\theta_{\mathbb{R}} \in \mathscr{E}$, so $\theta_{\mathbb{R}}$ restricts to an orthogonal operator on $U$. We shall be making a study of $\operatorname{det}\left(\theta_{\mathbb{R}}: U\right)$, the determinant of the action of $\theta_{\mathbb{R}}$ on $U$. The first step will be to pass to the complexifications. Noting that $\theta$ belongs to the algebra $\mathbb{C}_{\mathscr{E}}^{\mathscr{E}}=\operatorname{End}_{\mathbb{C} G}(\mathbb{C} X)$, we see that $\theta$ acts as on the $\mathbb{C}_{\mathscr{E}}$-submodule $\mathbb{C} U$ of $\mathbb{C} X$. 
There is a delicate matter concerning determinants which demands clarity even if at the risk of pedagogy. Again, consider finite-dimensional $\mathbb{C}$-vector space $S$ equipped with a non-degenerate symmetric bilinear form $\langle-\mid-\rangle$. One point of variance from the theory of inner product spaces is that not all of the subspaces of $S$ are normal (except in the trivial case $\operatorname{dim}_{\mathbb{C}}(S) \leq 1$ ). Recall that a subspace $R$ of $S$ is said to be normal provided the restriction of $\langle-\mid-\rangle$ to a symmetric bilinear form on $R$ is nondegenerate. This is equivalent to the condition that the subspace $R^{\perp}=$ $\{\rho \in S:\langle\rho \mid \sigma\rangle=0\}$ is complementary to $R$ in $S$. Given an orthogonal operator $\phi$ on $S$ then, with respect to any orthonormal basis, the matrices of $\phi$ and $\phi^{-1}$ are mutual transposes, and hence $\operatorname{det}(\phi)= \pm 1$. Supposing that $R$ is any subspace stabilized by $\phi$, then the determinant of $\phi$ on $R$, denoted $\operatorname{det}(\phi: R)$, need not be \pm 1 . (For a counterexample, consider the eigenspaces of the $\mathbb{C}$-linear extension of a rotation of the real plane.) However, if $R$ is a normal subspace of $S$, then the action of $\phi$ on $R$ is orthogonal, and we do have $\operatorname{det}(\phi: R)= \pm 1$.

Lemma 6.1. We have $\operatorname{det}\left(\theta_{\mathbb{R}}: U\right)=\operatorname{det}(\theta: \mathbb{C} U)= \pm 1$.

Proof. The subspace $\mathbb{C} U$ of $\mathbb{C} X$ is normal, because any orthonormal bases for $U$ is also an orthonormal basis for $\mathbb{C} U$. By comments above, $\operatorname{det}(\theta: \mathbb{C} U)= \pm 1$ and $\operatorname{det}\left(\theta_{\mathbb{R}}: \mathbb{C} U\right)=\operatorname{det}\left(\theta_{\mathbb{R}}: U\right)= \pm 1$. But $\theta$ and $\theta_{\mathbb{R}}$ belong to the same component of the group $O_{G}(\mathbb{C} X)$, which acts on $\mathbb{C} U$. By continuity, $\operatorname{det}\left(\theta_{\mathbb{R}}: \mathbb{C} U\right)=\operatorname{det}(\theta: \mathbb{C} U)$.

Lemma 6.2. There is a ring automorphism of $\mathbb{C} \mathscr{E}$ given by $\phi \mapsto \alpha_{\circ} \phi_{\circ} \alpha^{-1}$ for $\phi \in \mathscr{E}$.

Proof. By direct calculation,

$$
\alpha\left(\phi\left(\alpha\left(\sum_{x \in X} a_{x} x\right)\right)\right)=\sum_{y \in Y, x \in X} \alpha(\langle y|\phi| x\rangle) a_{x} y .
$$

So $\alpha_{\circ} \phi_{\circ} \alpha^{-1}$ is a $\mathbb{C}$-linear map. The rest is plain.

Lemma 6.3. The set $\alpha(\mathbb{C} U)$ is a $\mathbb{C} \mathscr{E}$-submodule of $\mathbb{C} X$. Allowing $\alpha$ to act as an automorphism of $\mathbb{C} \mathscr{E}$ as indicated in the latest lemma, $\alpha$ acts on the isomorphism classes of $\mathbb{C} \mathscr{E}$-modules, and this action sends the isomorphism class of $\mathbb{C} U$ to the isomorphism class of $\alpha(\mathbb{C} U)$.

Proof. Given any subset $S \subseteq \mathbb{C X}, \alpha$ sends the span of $S$ to the span of $\alpha(S)$. So $\alpha$ permutes the subspaces of $\mathbb{C} X$. Again, the rest is plain.

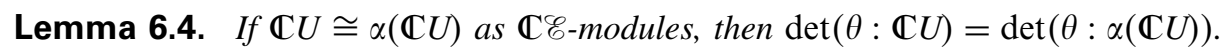

Proof. This is immediate from the fact that $\theta \in \mathbb{C}_{\mathscr{E}}$.

Let $V=\imath^{-1}(U)$ as a subspace of $\mathbb{R} Y$. Let $U$ and $v$ be orthonormal bases for $U$ and $V$, respectively. We extend $U$ and $v$ to orthonormal bases $\mathbb{U}$ and $\mathbb{V}$ for $\mathbb{R} X$ and $\mathbb{R} Y$. Of course, $\mathbb{R} X$ and $\mathbb{R} Y$ also have orthonormal bases $X$ and $Y$. Below, except where otherwise stated, the symbols $u, v, x, y$, sometimes with ornaments, denote arbitrary elements of $\mathbb{U}, \mathbb{V}, X, Y$. 
Lemma 6.5. With respect to the basis $\mathbb{U}$ of $\mathbb{C} X$, the matrix representing $\theta$ has $\left(u^{\prime}, u\right)$ entry

$$
\left\langle u^{\prime}|\theta| u\right\rangle=\sum_{v, v^{\prime}, u^{\prime \prime}}\left\langle u^{\prime}|\imath| v\right\rangle\left\langle v \mid \alpha\left(v^{\prime}\right)\right\rangle \alpha\left(\left\langle v^{\prime}\left|l^{-1}\right| u^{\prime \prime}\right\rangle\right)\left\langle\alpha\left(u^{\prime \prime}\right) \mid u\right\rangle
$$

Proof. Using the equality $u=\sum_{x}\langle x \mid u\rangle x$, a routine manipulation yields

$$
\theta(u)=\sum_{x} l_{\circ} \alpha_{\circ} l^{-1}{ }_{\circ} \alpha^{-1}(\langle x \mid u\rangle x)=\sum_{u^{\prime}, y, x}\left\langle u^{\prime}|\imath| y\right\rangle \alpha\left(\left\langle y\left|l^{-1}\right| x\right\rangle\right)\langle x \mid u\rangle u^{\prime} .
$$

In other words,

$$
\left\langle u^{\prime}|\theta| u\right\rangle=\sum_{y, x}\left\langle u^{\prime}|\imath| y\right\rangle \alpha\left(\left\langle y\left|l^{-1}\right| x\right\rangle\right)\langle x \mid u\rangle
$$

Since $\mathbb{V}$ is a orthonormal basis for $\mathbb{C} Y$, we have $\sum_{v}\langle v \mid v\rangle\left\langle v \mid v^{\prime}\right\rangle=\left\langle v \mid v^{\prime}\right\rangle$ for all $v, v^{\prime} \in \mathbb{C} Y$. (In the more sophisticated notation used by physicists and engineers, this is the "resolution of the identity operator," $\sum_{v}|v\rangle\langle v|=1$.) Similar comments hold for the orthonormal bases $\alpha(\mathbb{V})$ and $Y$ of $\mathbb{C} Y$ and for the orthonormal bases $\mathbb{U}$ and $\alpha(\mathbb{U})$ and $X$ of $\mathbb{C} X$. Hence,

$$
\left\langle u^{\prime}|\imath| y\right\rangle=\sum_{y^{\prime}, v}\left\langle u^{\prime}|\imath| y^{\prime}\right\rangle\left\langle y^{\prime} \mid v\right\rangle\langle v \mid y\rangle=\sum_{v}\left\langle u^{\prime}|\imath| v\right\rangle\langle v \mid y\rangle .
$$

Noting that $\left\langle\alpha\left(v^{\prime}\right) \mid y\right\rangle=\alpha\left(\left\langle v^{\prime} \mid y\right\rangle\right)$ and $\left\langle x\left|\alpha\left(u^{\prime \prime}\right)\right| u\right\rangle=\alpha\left(\left\langle x \mid u^{\prime \prime}\right\rangle\right)$, we obtain

$$
\langle v \mid y\rangle=\sum_{v^{\prime}}\left\langle v \mid \alpha\left(v^{\prime}\right)\right\rangle \alpha\left(\left\langle v^{\prime} \mid y\right\rangle\right), \quad\langle x \mid u\rangle=\sum_{u^{\prime \prime}} \alpha\left(\left\langle x \mid u^{\prime \prime}\right\rangle\right)\left\langle\alpha\left(u^{\prime \prime}\right) \mid u\right\rangle .
$$

Starting from the latest equation for $\left\langle u^{\prime}|\theta| u\right\rangle$, expanding the expression for $\left\langle u^{\prime}|\imath| y\right\rangle$ and then expanding the expressions for $\langle v \mid y\rangle$ and $\langle x \mid u\rangle$, we obtain

$$
\left\langle u^{\prime}|\theta| u\right\rangle=\sum_{v, v^{\prime}, y, x, u^{\prime \prime}}\left\langle u^{\prime}|\imath| v\right\rangle\left\langle v \mid \alpha\left(v^{\prime}\right)\right\rangle \alpha\left(\left\langle v^{\prime} \mid y\right\rangle\right) \alpha\left(\left\langle y\left|l^{-1}\right| x\right\rangle\right) \alpha\left(\left\langle x \mid u^{\prime \prime}\right\rangle\right)\left\langle\alpha\left(u^{\prime \prime}\right) \mid u\right\rangle .
$$

Using the fact that $\alpha$ preserves multiplication, then using the "resolution of the identity operator," we obtain the required formula.

Proposition 6.6. Let $\mu: \mathbb{C} X \rightarrow \mathbb{C} X$ and $o: \mathbb{C} X \rightarrow \mathbb{C} Y$ and $v: \mathbb{C} Y \rightarrow \mathbb{C} X$ be the $\mathbb{C}$ linear maps such that

$$
\left\langle u^{\prime \prime}|\mu| u\right\rangle=\left\langle\alpha\left(u^{\prime \prime}\right) \mid u\right\rangle, \quad\left\langle v^{\prime}|o| u^{\prime \prime}\right\rangle=\alpha\left(\left\langle v^{\prime}\left|l^{-1}\right| u^{\prime \prime}\right\rangle\right), \quad\left\langle v|v| v^{\prime}\right\rangle=\left\langle v \mid \alpha\left(v^{\prime}\right)\right\rangle .
$$

Then we have a commutative pentagon of $\mathbb{C}$-linear isomorphisms $\theta=l_{\circ} v_{\circ} o_{\circ} \mu$ as illustrated in the left-hand side diagram below. Furthermore, we have a commutative pentagon of $\mathbb{C}$-linear isomorphisms $\theta_{U}=l_{U \circ} v_{U \circ} o_{U \circ} \mu_{U}$ as illustrated in the right-hand side diagram, where $\theta_{U}, \imath_{U}, v_{U}, o_{U}, \mu_{U}$ are restrictions of $\theta, l, v, o, \mu$, and the domains and codomains of $\theta_{U}, \imath_{U}, v_{U}, o_{U}, \mu_{U}$ are as indicated in the diagram. 
Proof. The commutativity of the left-hand side diagram is immediate from the previous lemma. It remains only to show that $\theta, l, v, o, \mu$ restrict to isomorphisms with the specified domains and codomains. We first show that $\mu(\alpha(\mathbb{C} U)) \leq \mathbb{C} U$. The subspace $\mathbb{C} U$ is normal in $\mathbb{C} X$; indeed, the subspaces $\mathbb{C} U$ and $(\mathbb{C} U)^{\perp}$ are complementary in $\mathbb{C X}$ because they have orthonormal bases $\mathscr{U}$ and $\mathbb{U}-\mathcal{U}$, respectively. The functions $\left\langle u^{\prime \prime}|\mu|-\right\rangle$ and $\left\langle\alpha\left(u^{\prime \prime}\right) \mid-\right\rangle$ are $\mathbb{C}$-linear maps $\mathbb{C} X \rightarrow \mathbb{C}$, and they agree with each other on the basis $\mathbb{U}$, so $\left\langle u^{\prime \prime}|\mu|-\right\rangle=\left\langle\alpha\left(u^{\prime \prime}\right) \mid-\right\rangle$. In particular, $\left\langle u^{\prime \prime}|\mu| \alpha(u)\right\rangle=\alpha\left(u^{\prime \prime}\right)|\alpha(u)\rangle=\left\langle\alpha\left\langle u^{\prime \prime} \mid u\right\rangle=\delta_{u^{\prime \prime}, u}\right.$. So, when $u \in \mathscr{U}$, the $\mathbb{C}$ linear function $\langle-|\mu| \alpha(u)\rangle$ annihilates $(\mathbb{C} U)^{\perp}$. Hence, by the normality of $\mathbb{C} U$ in $\mathbb{C} X$, we have $\mu(\alpha(u)) \in \mathbb{C} U$. But $\alpha(\mathcal{U})$ is a $\mathbb{C}$-basis for $\alpha(\mathbb{C} U)$. We deduce that $\mu(\alpha(\mathbb{C} U)) \leq \mathbb{C} U$.

Two similar arguments show that $v(\mathbb{C} V) \leq \alpha(\mathbb{C} V)$ and $o_{U}(\mathbb{C} U) \leq \mathbb{C} V$. Since $\mu_{U}, o_{U}, v_{U}$ are restrictions of isomorphisms, they are injective. But the $\mathbb{C}$-vector spaces $\mathbb{C} U, \alpha(\mathbb{C} U), \mathbb{C} V, \alpha(\mathbb{C} V)$ all have the same dimension. So $\mu_{U}, o_{U}, v_{U}$ are isomorphisms with the specified domains and codomains. Since $\theta$ is a unit in $\mathbb{C}_{\mathscr{E}}$, the action of $\theta$ on $\mathbb{C} X$ restricts to a $\mathbb{C}$-linear automorphism $\theta_{U}$ on the $\mathbb{C}^{\mathscr{E}}$-submodule $\alpha(\mathbb{C} U)$. Finally, since $\alpha(\mathbb{C} V)=v_{U}\left(o_{U}\left(\mu_{U}(\alpha(\mathbb{C} U))\right)\right)$, we have $l(\alpha(\mathbb{C} V))=\theta(\alpha(\mathbb{C} U))=$ $\alpha(\mathbb{C} U)$; in other words, $l$ restricts to an isomorphism $\alpha(\mathbb{C} V) \rightarrow \alpha(\mathbb{C} U)$.
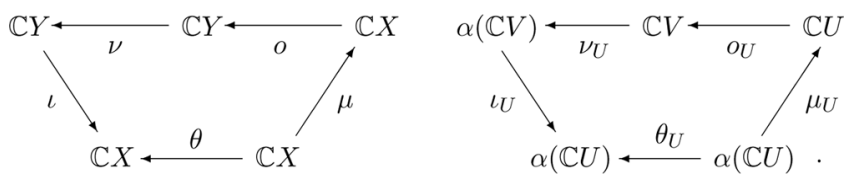

Some further notation for matrices will be convenient. Let $A, B, C$ be finitedimensional vector spaces with bases $\mathscr{A}, \mathscr{B}, \mathscr{C}$, respectively. Let $\phi: A \rightarrow B$ and $\psi$ : $B \rightarrow C$ be linear maps. We write $\langle\mathscr{B}|\phi| \mathscr{A}\rangle$ to denote the matrix representing $\phi$ with respect to the bases $\mathscr{B}$ and $\mathscr{A}$. Matrix multiplication is related to composition of linear maps via the formula $\langle\mathscr{C}|\psi \phi| \mathscr{A}\rangle=\langle\mathscr{C}|\psi| \mathscr{B}\rangle\langle\mathscr{B}|\phi| \mathscr{A}\rangle$. Suppose now that $A$, $B, C$ all have the same dimension and that the bases $\mathscr{A}, \mathscr{B}, \mathscr{C}$ are equipped with orderings (or, at least, with orderings well defined up to even permutations). Then the three matrices are square matrices and

$$
\operatorname{det}\langle\mathscr{C}|\psi \phi| \mathscr{A}\rangle=\operatorname{det}\langle\mathscr{C}|\psi| \mathscr{B}\rangle \cdot \operatorname{det}\langle\mathscr{B}|\phi| \mathscr{A}\rangle
$$

Note that, for these three determinants to be well defined, the orderings on the bases do need to be fixed (up to an even permutation) because, if we apply an odd permutation to one of the three orderings, then two of the determinants will be changed by a factor of -1 . Of course, when $A=B$, the $\operatorname{determinant} \operatorname{det}(\phi)=$ $\operatorname{det}\langle\mathscr{A}|\phi| \mathscr{A}\rangle$ is independent of $\mathscr{A}$.

We now impose arbitrarily chosen orderings $u_{1}<u_{2}<\cdots$ on the elements of $U$ and $v_{1}<v_{2}<\cdots$ on the elements of $v$.

Lemma 6.7. The isometry $l: \mathbb{R} Y \rightarrow \mathbb{R} X$ restricts to an isometry ${ }_{U} l: V \rightarrow U$. We have

$$
\operatorname{det}\left\langle u\left|o_{U}\right| U\right\rangle=\operatorname{det}\left\langle\left.\mathscr{U}\right|_{U} l \mid u\right\rangle= \pm 1
$$


Proof. The first sentence of the assertion is immediate from the definition of $V$. Since $U$ and $v$ are orthonormal bases for $U$ and $V$, we have $\operatorname{det}\left\langle U_{U} l \mid v\right\rangle= \pm 1$. The defining equation for $o_{U}$ can be rewritten as $\left\langle u\left|o_{U}\right| \mathcal{U}\right\rangle=\alpha\left\langle\left. u\right|_{U} l^{-1} \mid \mathcal{U}\right\rangle$. Therefore, $\operatorname{det}\left\langle\mathcal{U}\left|o_{U}\right| \mathcal{U}\right\rangle=\alpha\left(\left(\operatorname{det}\left\langle\left.\mathcal{U}\right|_{U} l \mid v\right\rangle\right)^{-1}\right)=\operatorname{det}\left\langle\left.\mathcal{U}\right|_{U} l \mid v\right\rangle$.

The proof of Proposition 2.2, in the next section, will be based on the following corollary to Proposition 6.6. Let us point out that, if our only aim were to present a proof of Proposition 2.2, then the hypothesis of the corollary could be imposed from the outset, and much the material above could be considerably simplified.

Corollary 6.8. If $\alpha(\mathbb{C} U)=\mathbb{C} U$ and $\alpha(\mathbb{C} V)=\mathbb{C} V$ as equalities of subspaces, then

$$
\operatorname{det}\left(\theta_{\mathbb{R}}: U\right)=\operatorname{det}\left(\theta_{U}\right)=\operatorname{det}\left(v_{U}\right) \cdot \operatorname{det}\left(\mu_{U}\right)
$$

Proof. By Lemma $6.1, \operatorname{det}\left(\theta_{\mathbb{R}}: U\right)=\operatorname{det}\left(\theta_{U}\right)$. The hypothesis on $\mathbb{C} U$ and $\mathbb{C} V$ implies that $v_{U}$ and $\mu_{U}$ are operators, so $v_{U}$ and $\mu_{U}$ have well defined determinants. By Proposition 6.6,

$$
\operatorname{det}\left(\theta_{U}\right)=\operatorname{det}\left\langle\mathscr{U}\left|\imath_{U}\right| v\right\rangle \cdot \operatorname{det}\left(v_{U}\right) \cdot \operatorname{det}\left\langle v\left|o_{U}\right| \mathcal{U}\right\rangle \cdot \operatorname{det}\left(\mu_{U}\right)
$$

The hypothesis on $\mathbb{C} U$ also implies that the $\mathbb{C}$-linear extension of ${ }_{U} l$ coincides with $\imath_{U}$, so the required equality now follows from Lemma 6.7 .

The proof of Theorem 2.3, in Section 8, will be based on the next corollary. Again, the corollary is obtained from Proposition 6.6 by applying determinants to the factorization of $\theta_{U}$. This time, though, we shall be considering different coordinate systems, and the full content of Proposition 6.6 will be needed. We order the elements of $\alpha(\mathcal{U})$ such that $\alpha\left(u_{1}\right)<\alpha\left(u_{2}\right)<\cdots$, likewise for $\alpha(v)$.

Corollary 6.9. If $\alpha(\mathbb{C} U) \cong \mathbb{C} U$ as an isomorphism of $\mathbb{C} \mathscr{E}$-modules, then

$$
\operatorname{det}\left(\theta_{\mathbb{R}}: U\right)=\operatorname{det}\left(\theta_{U}\right)=\operatorname{det}\left\langle\alpha(U)\left|l_{U}\right| \alpha(v)\right\rangle \cdot \operatorname{det}\left\langle\left. U\right|_{U} l \mid v\right\rangle .
$$

Proof. By Lemmas 6.1 and $6.4, \quad \operatorname{det}\left(\theta_{\mathbb{R}}: U\right)=\operatorname{det}\left(\theta_{\mathbb{C}}: U\right)=\operatorname{det}\left(\theta_{U}\right) . \quad$ By Proposition 6.6,

$$
\operatorname{det}\left(\theta_{U}\right)=\operatorname{det}\left\langle\alpha(\mathcal{U})\left|l_{U}\right| \alpha(v)\right\rangle \cdot \operatorname{det}\left\langle\alpha(v)\left|v_{U}\right| v\right\rangle \cdot \operatorname{det}\left\langle v\left|o_{U}\right| \mathcal{U}\right\rangle \cdot \operatorname{det}\left\langle\mathscr{U}\left|\mu_{U}\right| \alpha(\mathcal{U})\right\rangle .
$$

The proof of Proposition 6.6 shows that, given $u_{j}, u_{i} \in \mathcal{U}$, then $\left\langle u_{i}\left|\mu_{U}\right| u_{j}\right\rangle=\delta_{i, j}$. In other words, $\left\langle\mathscr{U}\left|\mu_{U}\right| \alpha(\mathcal{U})\right\rangle$ is the identity matrix. In particular, $\operatorname{det}\langle\mathscr{U}|\mu| \alpha(\mathscr{U})\rangle=1$. A similar argument yields $\left\langle\alpha\left(v_{i}\right)\left|v_{U}\right| v_{j}\right\rangle=\delta_{i, j}$ and $\operatorname{det}\left\langle\alpha(v)\left|v_{U}\right| v\right\rangle=1$. Again, an appeal to Lemma 6.7 completes the argument.

At the end of this article, we shall explain why the hypothesis $\alpha(\mathbb{C} U) \cong \mathbb{C} U$ is necessary. 


\section{THE TORNEHAVE MORPHISM}

We have now assembled more than enough material to recover Tornehave's result, Proposition 2.2. We shall also establish the Tornehave morphism as an inflaky morphism $K \rightarrow B^{\times}$. As before, let $\kappa \in K(G)$, and let $\left(X, Y, \imath, \theta_{\mathbb{R}}\right)$ be a choice tuple for a zombie module $W$ for $\kappa$.

Proof of Proposition 2.2. In view of the definitions of $\overline{\operatorname{die}}_{G}$ and $\overline{\operatorname{torn}}_{G}^{\pi}$, we are required to show that, given $I \leq G$,

$$
\operatorname{dim}_{\mathbb{R}}\left(W^{I}\right) \stackrel{\text { par }}{=} \sum_{\mathscr{O} \in I \backslash X} \log _{\pi}|\mathscr{O}|-\sum_{\mathscr{G} \in I \backslash Y} \log _{\pi}|\mathscr{O}| .
$$

Applying Proposition 4.4 with the substitutions $M=\mathbb{R} Y$ and $\phi=\theta_{\mathbb{R}}$, we have

$$
\operatorname{par}\left(\operatorname{dim}_{\mathbb{R}}\left(W^{I}\right)\right)=\operatorname{par}\left[\operatorname{deg}_{\mathbb{R} X}\left(\theta_{\mathbb{R}}\right) @ I\right]=\operatorname{det}\left(\theta_{\mathbb{R}}:(\mathbb{R} X)^{I}\right) .
$$

Let $U=(\mathbb{R} X)^{I}$ as an $\mathscr{E}$-submodule of $\mathbb{R} X$. Then $\mathbb{C} U=(\mathbb{C} X)^{I}$. Since $\imath$ is a $G$ invariant isometry, $V=l^{-1}(U)=(\mathbb{R} Y)^{I}$ and $\mathbb{C} V=(\mathbb{C} Y)^{I}$. The actions of $\alpha$ on $\mathbb{C} X$ and $\mathbb{C} Y$ commute with the actions of $I$, hence $\alpha(\mathbb{C} U)=\mathbb{C} U$ and $\alpha(\mathbb{C} V)=\mathbb{C} V$. So Corollary 6.8 applies, and

$$
\operatorname{par}\left(\operatorname{dim}_{\mathbb{R}}\left(W^{I}\right)\right)=\operatorname{det}\left(\theta_{\mathbb{R}}: U\right)=\operatorname{det}\left(v_{U}\right) \cdot \operatorname{det}\left(\mu_{U}\right) .
$$

Let $\mathscr{O}_{1}, \mathscr{O}_{2}, \ldots$ be the $I$-orbits in $X$. Put

$$
u_{j}=\frac{1}{\sqrt{\left|\mathscr{G}_{j}\right|}} \sum_{x \in \mathscr{G}_{j}} x
$$

then $\mathscr{U}=\left\{u_{1}, u_{2}, \ldots\right\}$ is an orthonormal basis for $U$. Let $\delta_{j}=\log _{\pi}\left|\mathscr{O}_{j}\right|$. We have $\alpha\left(\sqrt{\left|\mathscr{O}_{j}\right|}\right)=\operatorname{par}\left(\delta_{j}\right) \sqrt{\left|O_{j}\right|}$; hence, $\alpha\left(u_{j}\right)=\operatorname{par}\left(\delta_{j}\right) u_{j}$. So the matrix representing $\mu_{U}$ with respect to $\mathcal{U}$ is the diagonal matrix with $(j, j)$-entry $\left\langle u_{j}|\rho| u_{j}\right\rangle=\left\langle\alpha\left(u_{j}\right) \mid u_{j}\right\rangle=$ $\operatorname{par}\left(\delta_{j}\right)$. Hence $\operatorname{det}\left(\mu_{U}\right)=\prod_{j} \operatorname{par}\left(\delta_{j}\right)=\operatorname{par}\left(\sum_{\mathscr{G} \in I \backslash X} \log _{\pi}|\mathscr{O}|\right)$. A similar argument applied to the $I$-orbits of $Y$ yields $\operatorname{det}\left(v_{U}\right)=\operatorname{par}\left(\sum_{\mathscr{G} \in I \backslash Y} \log _{\pi}|\mathscr{O}|\right)$. Comparing with the formula at the end of the previous paragraph, we obtain the required congruence.

Lemma 7.1. Let $H \leq G \unrhd N$ and let $\phi: G \stackrel{\sim}{\rightarrow} F$ be a group isomorphism.

(1) The $\mathbb{R} H$-module $\operatorname{Res}_{H, G}(W)$ and the $\mathbb{R} F$-module $\operatorname{Iso}_{F, G}^{\phi}(W)$ are zombie modules for $\operatorname{res}_{H, G}(\kappa)$ and $\inf _{G, G / N}(\kappa)$.

(2) Given a zombie $\mathbb{R} H$-module $W^{\prime}$ for an element $\kappa^{\prime} \in K(H)$, then $\operatorname{Ind}_{G, H}\left(W^{\prime}\right)$ is a zombie module for ind $_{G, H}\left(\kappa^{\prime}\right)$.

(3) Given a zombie $\mathbb{R} G / N$-module $W^{\prime \prime}$ for an element $\kappa^{\prime \prime} \in K(G / N)$, then $\operatorname{Inf}_{G, G / N}\left(W^{\prime \prime}\right)$ is a zombie module for $\inf _{G, G / N}\left(\kappa^{\prime \prime}\right)$.

Proof. Let $\left(X^{\prime}, Y^{\prime}, \imath^{\prime}, \theta_{\mathbb{R}}^{\prime}\right)$ be a choice tuple for $W^{\prime}$ as a zombie module for $\kappa^{\prime}$. Let $X=\operatorname{Ind}_{G, H}\left(X^{\prime}\right)$ and $Y=\operatorname{Ind}_{G, H}\left(Y^{\prime}\right)$. Then $\mathbb{C} X=\operatorname{Ind}_{G, H}\left(\mathbb{C} X^{\prime}\right)=\bigoplus_{g H \subseteq G} g . \mathbb{C} X^{\prime}$ 
as a direct sum of mutually orthogonal subspaces, and similarly for $\mathbb{C} Y$ and $\mathbb{C} Y^{\prime}$. Consider the maps

$\operatorname{tr}_{G, H}: \operatorname{Hom}_{\mathbb{C} H}\left(\mathbb{C} X^{\prime}, \mathbb{C} Y^{\prime}\right) \rightarrow \operatorname{Hom}_{\mathbb{C} G}(\mathbb{C} X, \mathbb{C} Y), \operatorname{tr}_{G, H}: \operatorname{End}_{\mathbb{C} H}\left(\mathbb{C} X^{\prime}\right) \rightarrow \operatorname{End}_{\mathbb{C} G}(\mathbb{C} X)$

given by $\operatorname{tr}_{G, H}(\psi)=\sum_{g H \subseteq G} g \psi g^{-1}$. (These are the relative trace maps, but with restricted domains.) Let $\imath=\operatorname{tr}_{G, H}\left(l^{\prime}\right)$ and $\theta_{\mathbb{R}}=\operatorname{tr}_{G, H}\left(\theta_{\mathbb{R}}^{\prime}\right)$. Noting that $\operatorname{tr}_{G, H}$ sends paths in $O_{H}\left(\mathbb{C} X^{\prime}\right)$ to paths in $O_{G}(\mathbb{C} X)$, we see that $\left(X, Y, \imath, \theta_{\mathbb{R}}\right)$ is a choice tuple for $\operatorname{Ind}_{G, H}\left(W^{\prime}\right)$ as a zombie module for $\operatorname{ind}_{G, H}\left(\kappa^{\prime}\right)$. Part (2) is now established. Parts (1) and (3) are similar and easier.

Corollary 7.2. The formula for $\left[\overline{\operatorname{torn}}_{G}^{\pi}(\kappa) @ I\right]$, given in Section 3, specifies a well defined linear map $\overline{\operatorname{torn}}_{G}^{\pi}: K(G) \rightarrow B^{\times}(G)$. The maps $\overline{\operatorname{torn}}_{G}^{\pi}$ are the coordinate maps of an inflaky morphism $\overline{\text { torn }}^{\pi}: K \rightarrow B^{\times}$.

Proof. The formula specifies $\overline{\operatorname{torn}}_{G}^{\pi}(\kappa)$ as an element of $\beta(G)$ and in terms of $G$ sets $X$ and $Y$. It is easy to check that the value of the expression for $\left[\overline{\operatorname{torn}}_{G}^{\pi}(\kappa) @ I\right]$ depends only on $\kappa$ and not on the choice of $X$ and $Y$. By Proposition 2.2, $\overline{\operatorname{torn}}_{G} \pi(\kappa) \in$ $B^{\times}(G)$. The commutativity with induction, restriction, isogation, and inflation was established in Lemma 7.1.

The use of Lemma 7.1, here, can be avoided. Instead, the commutativity with those four elemental maps can be proved by direct manipulation of formulas. Let us mention that, for commutativity with induction, the manipulations are not entirely straightforward. This alternative line of argument is used in [3, Section 4] to show that the lifted Tornehave maps $\operatorname{torn}_{G}^{\pi}: K(G) \rightarrow B^{*}(G)$ are the coordinate maps of an inflaky morphism $\overline{\operatorname{torn}}^{\pi}: K \rightarrow B^{*}$.

\section{THE ZOMBIE MORPHISM}

We now prove Theorem 2.3. Let $\kappa, X, Y, \imath, \theta, \theta_{\mathbb{R}}, W$ be as before.

Lemma 8.1. The element ori $_{G}[W] \in O_{\mathbb{R}}(G)$ depends only on $\kappa$ and $\alpha$, not on the choice tuple $\left(X, Y, \imath, \theta_{\mathbb{R}}\right)$.

Proof. We fix $\kappa$ and $\alpha$. For an absolutely irreducible $\mathbb{R} G$-character $\chi$, we must show that the $\chi$ th coordinate $\left[\chi @ \operatorname{ori}_{G}[W]\right]$ is independent of $\left(X, Y, \imath, \theta_{\mathbb{R}}\right)$. In the notation of Section 4 , put $M=\mathbb{R} X$ and $\phi=\theta_{\mathbb{R}}$ and $U=U_{\chi}^{\mathrm{Gal}}$. By Proposition 4.6 and Remark 4.7,

$$
\operatorname{par}\left[\chi @ \operatorname{ori}_{G}[W]\right]=\operatorname{par}\left[\chi @ \operatorname{ori}_{\mathbb{R} X}\left(\theta_{\mathbb{R}}\right)\right]=\operatorname{det}\left(\theta_{\mathbb{R}}: U\right) .
$$

The $\mathbb{C} \mathscr{E}$-module $\mathbb{C} U$ is isomorphic to the direct sum of the Galois conjugates of the simple $\mathbb{C} \mathscr{E}$-module $\mathbb{C} U_{\chi}$, so $\alpha(\mathbb{C} U) \cong \mathbb{C} U$ and Corollary 6.9 applies. Hence

$$
\operatorname{par}\left[\chi @ \operatorname{ori}_{G}[W]\right]=\operatorname{det}\left\langle\alpha(\mathcal{U})\left|l_{U}\right| \alpha(v)\right\rangle \cdot \operatorname{det}\left\langle\left.\mathcal{U}\right|_{U} l \mid v\right\rangle .
$$

In particular, fixing $X, Y, l$, the elements $\operatorname{par}\left[\chi @ \operatorname{ori}_{G}[W]\right] \in \mathbb{Z}^{\times}$and $\left[\chi @\right.$ ori $\left._{G}[W]\right] \in$ $\mathbb{F}_{2}$ are independent of $\theta_{\mathbb{R}}$. 
Still fixing $X$ and $Y$, we now show independence from the choice of $l$. Let $\iota^{\prime}$ be another $G$-invariant isometry $\mathbb{R} Y \rightarrow \mathbb{R} X$. Then $\imath^{\prime}=\sigma_{\circ} l$ for some $\sigma \in O_{G}(\mathbb{R} X)$. Extending $\mathbb{C}$-linearly and then restricting $\sigma$ to $G$-invariant operators $\sigma_{U}$ on $\alpha(\mathbb{C} U)$ and ${ }_{U} \sigma$ on $\mathbb{C} U$, the operators $\sigma_{U}$ and ${ }_{U} \sigma$ are orthogonal because, as we saw in the proof of Proposition 6.6, $\alpha(\mathbb{C} U)$ and $\mathbb{C} U$ are normal subspaces of $\mathbb{C} X$ (they have orthonormal bases $\alpha(\mathcal{U})$ and $U$ ). So $\operatorname{det}\left(\sigma_{U}\right)= \pm 1$ and $\operatorname{det}\left({ }_{U} \sigma\right)= \pm 1$. But $\alpha(\mathbb{C} U) \cong \mathbb{C} U$ and $\sigma \in \mathbb{C} \mathscr{E}$, so the actions of $\sigma$ on $\alpha(\mathbb{C} U)$ and on $\mathbb{C} U$ have the same determinant, $\operatorname{det}\left(\sigma_{U}\right)=\operatorname{det}\left({ }_{U} \sigma\right)$. Therefore, $\operatorname{det}\left(\sigma_{U}\right) \cdot \operatorname{det}\left({ }_{U} \sigma\right)=1$ and

$$
\begin{aligned}
& \operatorname{det}\left\langle\alpha(U)\left|l_{U}^{\prime}\right| \alpha(v)\right\rangle \cdot \operatorname{det}\left\langle\left. U\right|_{U} \imath^{\prime} \mid v\right\rangle \\
& =\operatorname{det}\left\langle\alpha(U)\left|\sigma_{U}\right| \alpha(U)\right\rangle \cdot \operatorname{det}\left\langle\alpha(U)\left|l_{U}\right| \alpha(v)\right\rangle \cdot \operatorname{det}\left\langle\left. U\right|_{U} \sigma \mid U\right\rangle \cdot \operatorname{det}\left\langle\left.\mathscr{U}\right|_{U} l \mid v\right\rangle \\
& =\operatorname{det}\left(\sigma_{U}\right) \cdot \operatorname{det}\left\langle\alpha(U)\left|l_{U}\right| \alpha(v)\right\rangle \cdot \operatorname{det}\left({ }_{U} \sigma\right) \cdot \operatorname{det}\left\langle\left.\mathscr{U}\right|_{U} l \mid v\right\rangle \\
& =\operatorname{det}\left\langle\alpha(U)\left|l_{U}\right| \alpha(v)\right\rangle \cdot \operatorname{det}\left\langle\left. U\right|_{U} l \mid v\right\rangle .
\end{aligned}
$$

We have shown that, fixing $X$ and $Y$, then $\left[\chi @ \operatorname{ori}_{G}[W]\right]$ is independent of $l$.

Finally, we show independence from the choices of $X$ and $Y$. Let $X_{0}$ and $Y_{0}$ be the $G$-sets, unique up to isomorphism, such that $\kappa=\left[X_{0}\right]-\left[Y_{0}\right]$ and no isomorphism class of transitive $G$-sets occurs in both $X_{0}$ and $Y_{0}$. Then $X \cong X_{0} \sqcup Z$ and $Y \cong Y_{0} \sqcup Z$ for some $G$-set $Z$. Let $\theta_{0}, \theta_{0, \mathbb{R}}, W_{0}$ be constructed from a $G$-invariant isometry $\imath_{0}: \mathbb{R} Y_{0} \rightarrow \mathbb{R} X_{0}$ in the same way that $\theta, \theta_{\mathbb{R}}, W$ were constructed from l. Our argument will be complete when we have shown that $\operatorname{ori}_{G}\left[W_{0}\right]=\operatorname{ori}_{G}[W]$. Making the identifications $\mathbb{R} X=\mathbb{R} X_{0} \oplus \mathbb{R} Z$ and $\mathbb{R} Y=\mathbb{R} Y_{0} \oplus \mathbb{R} Z$, we can extend $\imath_{0}$ to an isometry $\imath^{\prime}: \mathbb{R} Y \rightarrow \mathbb{R} Z$ which acts as the identity operator on $\mathbb{R} Z$. Let $\theta^{\prime}$, $\theta_{\mathbb{R}}^{\prime}, W^{\prime}$ be constructed from $\imath^{\prime}$ as $\theta, \theta_{\mathbb{R}}, W$ were constructed from $\imath$. Then $\theta^{\prime}$ acts as $\theta_{0}$ on $\mathbb{R} X_{0}$ and as the identity on $\mathbb{R} Z$. By the independence from the choice of the deformation $\theta_{\mathbb{R}}$, we may assume that $\theta_{\mathbb{R}}^{\prime}$ acts as $\theta_{0, \mathbb{R}}$ on $\mathbb{R} X_{0}$ and as the identity on $\mathbb{R} Z$. Then $W^{\prime}=W_{0}$. By the independence from the choice of $l$, we have $\operatorname{ori}_{G}\left[W^{\prime}\right]=$ $\operatorname{ori}_{G}[W]$.

All the components of Theorem 2.3 have now been established, and it remains only to put them together. Lemma 8.1 says that there exists a well defined $\mathbb{Z}$-linear

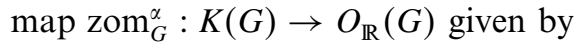

$$
\operatorname{zom}_{G}^{\alpha}(\kappa)=\operatorname{ori}_{G}[W]
$$

Lemma 7.1 implies that the maps $\operatorname{zom}_{G}^{\alpha}$ are the coordinate maps of an inflaky morphism zom $\alpha: K \rightarrow O_{\mathbb{R}}$. Proposition 2.2 implies that

$$
\underline{\operatorname{die}}_{G}\left(\operatorname{zom}_{G}^{\alpha}(\kappa)\right)=\underline{\operatorname{die}}_{G}[W]=\overline{\operatorname{torn}}_{G}^{\pi}(\kappa) .
$$

Therefore, $\overline{\operatorname{torn}}^{\pi}=$ die $_{\mathrm{o}} \mathrm{zom}^{\alpha}$.

\section{EXAMPLES AND PROPERTIES}

We shall give some examples, and then we shall use them to show that $\pi$ uniquely determines the reduced Tornehave morphism $\overline{\operatorname{torn}}$. 
Calculating the values of $\overline{\operatorname{torn}}_{G}^{\pi}$ is straightforward. Indeed, in Section 3, we defined $\overline{\operatorname{torn}}_{G}^{\pi}$ by a formula for the Ith coefficient of $\overline{\operatorname{torn}}_{G}^{\pi}(\kappa)$, where $I \leq G$ and $\kappa \in K(G)$. The following remark expresses that formula in a manner that is often convenient when examining concrete examples.

Remark 9.1. Let $I \leq G$, and let $t_{I}^{\pi}: B(G) \rightarrow \mathbb{Z}$ be the linear map such that

$$
t_{I}^{\pi}[G / U]=\sum_{I g U \subseteq G} \log _{\pi} \frac{|\operatorname{Ig} U|}{|U|}
$$

for $U \leq G$. That is to say, letting $|G: U|=r_{1} .1+r_{2} .2+\ldots$ be the integer partition such that $r_{i}$ is the number of $I$-orbits with size $i$ in $G / U$, then

$$
t_{I}^{\pi}[G / U]=r_{1} \log _{\pi}(1)+r_{2} \log _{\pi}(2)+\cdots
$$

Given $\kappa \in K(G)$, then $\left[\overline{\operatorname{torn}}_{G}^{\pi}(\kappa) @ I\right] \stackrel{\text { par }}{=} t_{I}^{\pi}(\kappa)$.

It would be desirable to have a similar formula for the $\chi$ th coordinate of $\operatorname{zom}_{G}^{\alpha}(\kappa)$, where $\chi \in \operatorname{Abs} \operatorname{Irr}(\mathbb{R} G)$. The next result supplies such a formula, but only in a special case.

Proposition 9.2. Let $\chi$ be an absolutely irreducible linear $\mathbb{R} G$-character, in other words, $\chi$ is an irreducible $\mathbb{R} G$-character such that the kernel $\operatorname{Ker}(\chi)$ has index 1 or 2 in $G$. Let $z_{\chi}^{\pi}: B(G) \rightarrow \mathbb{Z}$ be the linear map such that

$$
z_{\chi}^{\pi}[G / U]= \begin{cases}\log _{\pi}|G: U| & \text { if } U \leq \operatorname{Ker}(\chi) \\ 0 & \text { otherwise. }\end{cases}
$$

Then $\left[\chi @ \operatorname{zom}_{G}^{\alpha}(\kappa)\right] \stackrel{\text { par }}{=} z_{\chi}^{\pi}(\kappa)$.

Proof. Write $K=\operatorname{Ker}(\chi)$. Let $S$ be a simple $\mathbb{R} G$-module with character $\chi$. A Frobenius Reciprocity argument shows that, given a subgroup $U \leq G$, then $S$ occurs in $\mathbb{R} G / U$ if and only if $K \leq U$. Moreover, in that case, $S$ has multiplicity 1 in $\mathbb{R} G / U$.

Let $e$ be the primitive idempotent of $Z(\mathbb{R} G)$ such that $e S=S$. Write $\kappa=[X]-$ $[Y]$ with $X=G / A_{1} \sqcup G / A_{2} \sqcup \ldots$ and $Y=G / B_{1} \sqcup G / B_{2} \sqcup \ldots$ as disjoint unions of transitive $G$-sets. The observation in the previous paragraph implies that, letting $m$ be the multiplicity of $\chi$ in the $\mathbb{R} G$-module $\mathbb{R} X \cong \mathbb{R} Y$, we can number the subgroups $A_{i}$ such that $S$ occurs in $A_{i}$ if and only if $i \leq m$. We can number the subgroups $B_{i}$ similarly. Then $e \mathbb{R} X=e \mathbb{R} G / A_{1} \oplus \cdots \oplus e \mathbb{R} G / A_{m}$ as a direct sum of copies of $S$. The $\mathbb{R} G$-module $e \mathbb{R} Y$ decomposes likewise. For each $1 \leq i \leq m$, let

$$
u_{i}=\frac{1}{\sqrt{\left|G: A_{i}\right|}} \sum_{g A_{i} \subseteq G} \chi\left(g^{-1}\right) g A_{i}, \quad v_{i}=\frac{1}{\sqrt{\left|G: B_{i}\right|}} \sum_{g B_{i} \subseteq G} \chi\left(g^{-1}\right) g B_{i} .
$$


Since $|G| e=\sum_{g \in G} \chi\left(g^{-1}\right) g$, the sets $\left\{u_{1}, \ldots, u_{m}\right\}$ and $\left\{v_{1}, \ldots, v_{m}\right\}$ are orthonormal bases for $e \mathbb{R} X$ and $e \mathbb{R} Y$, respectively. We have

$$
\alpha\left(u_{i}\right)=\operatorname{par}\left(\log _{\pi}\left|G: A_{i}\right|\right) u_{i} \quad \alpha\left(v_{i}\right)=\operatorname{par}\left(\log _{\pi}\left|G: B_{i}\right|\right) v_{i} .
$$

In the notation of Section 6 , put $U=e \mathbb{R} X$. Then $V=e \mathbb{R} Y$. Plainly, $\alpha(\mathbb{C} U)=$ $e \mathbb{C} X=\mathbb{C} U$ and $\alpha(\mathbb{C} V)=\mathbb{C} V$, so Corollary 6.8 applies. We have

$$
\operatorname{det}\left(\mu_{U}\right)=\prod_{i=1}^{m} \operatorname{par}\left(\log _{\pi}\left|G: A_{i}\right|\right)
$$

and similarly for $v_{U}$ and the subgroups $B_{i}$. Therefore,

$$
\operatorname{det}\left(\theta_{\mathbb{R}}: U\right)=\operatorname{par}\left(\sum_{i=1}^{m}\left(\log _{\pi}\left|G: A_{i}\right|-\log _{\pi}\left|G: B_{i}\right|\right)\right)=\operatorname{par}\left(z_{\chi}^{\pi}(\kappa)\right) .
$$

On the other hand, since $S$ is 1-dimensional and stable under Galois automorphisms,

$$
\left[\chi @ \operatorname{zom}_{G}^{\alpha}(\kappa)\right] \stackrel{\text { par }}{=} \operatorname{dim}_{\mathbb{R}}(U \cap W) .
$$

So $\operatorname{par}\left[\chi @ \operatorname{zom}_{G}^{\alpha}(\kappa)\right]=\operatorname{par}\left(\operatorname{dim}_{\mathbb{R}}(U \cap W)\right)=\operatorname{det}\left(\theta_{\mathbb{R}}: U\right)$.

A refinement of the argument shows that, in fact, there exists a zombie module $W$ for $\kappa$ such that, for any linear $\mathbb{C} G$-character $\chi$, the multiplicity of $\chi$ in $W$ has the same parity as $z_{\chi}^{\pi}(\kappa)$, where $z_{\chi}^{\pi}(\kappa)$ is defined by the same formula as above.

Example 9.3. Suppose that $G=V_{4}$, the Klein four-group. Then $K(G)$ is a free cyclic $\mathbb{Z}$-module generated by the element $2 e_{G}^{G}=[G / 1]-[G / X]-[G / Y]-[G / Z]+$ $2[G / G]$ where $X, Y, Z$ are the three proper subgroups of $G$. We have

$$
\overline{\operatorname{torn}}_{G}^{\pi}\left(2 e_{G}^{G}\right)= \begin{cases}1-2 e_{G}^{G} & \text { if } 2 \in \pi \\ 1 & \text { otherwise }\end{cases}
$$

Letting $\chi_{0}, \ldots, \chi_{3}$ be the irreducible $\mathbb{R} G$-characters, then $\left\{\operatorname{ori}_{G}\left(\chi_{0}\right), \ldots\right.$, ori $\left.{ }_{G}\left(\chi_{3}\right)\right\}$ is an $\mathbb{F}_{2}$-basis for $O_{\mathbb{R}}(G)$ and

$$
\operatorname{zom}_{G}^{\alpha}\left(2 e_{G}^{G}\right)= \begin{cases}\operatorname{ori}_{G}\left(\chi_{0}+\chi_{1}+\chi_{2}+\chi_{3}\right) & \text { if } 2 \in \pi \\ 0 & \text { otherwise }\end{cases}
$$

Proof. The argument is similar to the proof of Example 9.6 below. In fact, it is easier, because all the irreducible $\mathbb{R} G$-characters are linear. So let us omit the details.

In [3], it turns out that, for $p$-groups, the theory of the lifted Tornehave morphism torn ${ }^{\{p\}}: K \rightarrow B^{*}$ is much the same in the case $p \neq 2$ as it is in the case $p=2$. However, the reduced Tornehave morphism $\overline{\operatorname{torn}}^{\{p\}}$ vanishes on $p$ groups when $p \neq 2$. In fact, the next remark tells us that all the reduced Tornehave morphisms vanish on groups with odd order. 
Remark 9.4. Supposing that $|G|$ is odd, then $\operatorname{zom}_{G}^{\alpha}$ and $\overline{\operatorname{torn}}_{G}^{\pi}$ are the zero maps.

Proof. For a contradiction, suppose that $\operatorname{zom}_{G}^{\alpha}(\kappa) \neq 0$ for some $\kappa \in K(G)$. The trivial $\mathbb{R} G$-character $\chi_{0}$ is the unique absolutely irreducible $\mathbb{R} G$-character, so $O_{\mathbb{R}}(G)=\left\{0, \operatorname{ori}_{G}\left(\chi_{0}\right)\right\}$ and $\operatorname{zom}_{G}^{\alpha}(\kappa)=\operatorname{ori}_{G}\left(\chi_{0}\right)$. Let $C$ be a cyclic subgroup of $G$, and let $\psi_{0}$ be the trivial $\mathbb{R} C$-character. Since $K(C)=0$, we have

$$
0=\operatorname{zom}_{C}^{\alpha}\left(\operatorname{res}_{C, G}(\kappa)\right)=\operatorname{res}_{C, G}\left(\operatorname{zom}_{G}^{\alpha}(\kappa)\right)=\operatorname{ori}_{G}\left(\operatorname{res}_{C, G}\left(\chi_{0}\right)\right)=\operatorname{ori}_{G}\left(\psi_{0}\right) \neq 0 .
$$

With this contradiction, we have shown that $\operatorname{zom}_{G}^{\alpha}=0$. Hence $\overline{\operatorname{torn}}_{G}^{\pi}=$ $\underline{\operatorname{die}}_{G \text { o }} \operatorname{zom}_{G}^{\alpha}=0$.

The following obvious remark gives another sufficient criterion for the vanishing of $\overline{\operatorname{torn}}_{G}^{\pi}$.

Remark 9.5. We have $\overline{\operatorname{torn}}_{G}^{\pi}=\sum_{p} \overline{\operatorname{torn}}_{G}^{\{p\}}$ where $p$ runs over those prime divisors of $|G|$ which belong to $\pi$. In particular, if no prime divisor of $|G|$ belongs to $\pi$, then $\overline{\operatorname{torn}}_{G}^{\pi}=0$.

Example 9.6. Suppose that $p$ is odd and $G=D_{2 p}$, the dihedral group with order $2 p$. Then $K(G)$ is a free cyclic $\mathbb{Z}$-module generated by the element $2 e_{G}^{G}=[G / 1]-$ $2[G / B]-[G / A]+2[G / G]$ where $B$ and $A$ are subgroups with orders 2 and $p$, respectively. We have

$$
\overline{\operatorname{torn}}_{G}^{\pi}\left(2 e_{G}^{G}\right)= \begin{cases}1-2 e_{G}^{G} & \text { if } p \in \pi, \\ 1 & \text { otherwise. }\end{cases}
$$

Let $\chi_{0}$ be the trivial $\mathbb{R} G$-character, let $\chi_{1}$ be the nontrivial linear $\mathbb{R} G$-character, and let $\chi_{2}$ be a faithful irreducible $\mathbb{R} G$-character. (Recall that $\chi_{2}$ arises from an action of $G$ on a $p$-gon.) Then $\left\{\operatorname{ori}_{G}\left(\chi_{0}\right)\right.$, ori ${ }_{G}\left(\chi_{1}\right)$, ori $\left.{ }_{G}\left(\chi_{2}\right)\right\}$ is an $\mathbb{F}_{2}$-basis for $O_{\mathbb{R}}(G)$ and

$$
\operatorname{zom}_{G}^{\alpha}\left(2 e_{G}^{G}\right)= \begin{cases}\operatorname{ori}_{G}\left(\chi_{0}+\chi_{1}+\chi_{2}\right) & \text { if } p \in \pi, \\ 0 & \text { otherwise. }\end{cases}
$$

Proof. Since $G$ is the unique noncyclic subgroup of $G$, we have $\mathbb{Q} K(G)=\mathbb{Q} e_{G}^{G}$. The equation for $2 e_{G}^{G}$ can be obtained in various ways, for instance, by using Gluck's Idempotent Formula (see Section 3). It follows that $K(G)=\mathbb{Z} e_{G}^{G}$.

By Remark 9.5, we need only establish the equation for $\overline{\operatorname{torn}}_{G}^{\pi}\left(2 e_{G}^{G}\right)$ in the two cases $\pi=\{p\}$ and $\pi=\{2\}$. The values of $t_{I}^{\{p\}}[G / U]$ and $t_{I}^{\{2\}}[G / U]$, calculated using Remark 9.1, are as shown in the first pair of tables below. For instance, in the case $I=U=B$, the integer partition in Remark 9.1 is $|G: U|=p=1.1+$ $(p-1) .2$. Hence $t_{I}^{\pi}[G / U]=\log _{\pi}(1)+(p-1) \log _{\pi}(2)=(p-1) \log _{\pi}(2)$, which is 0 or $p-1$, respectively, when $\pi$ is $\{p\}$ or $\{2\}$. Modulo 2 , we have

$$
\left(\begin{array}{llll}
0 & 0 & 0 & 0 \\
0 & 0 & 0 & 0 \\
2 & 1 & 0 & 0 \\
1 & 1 & 0 & 0
\end{array}\right)\left(\begin{array}{c}
1 \\
-2 \\
-1 \\
2
\end{array}\right) \equiv\left(\begin{array}{l}
0 \\
0 \\
0 \\
1
\end{array}\right), \quad\left(\begin{array}{cccc}
0 & 0 & 0 & 0 \\
p & p-1 & 1 & 0 \\
0 & 0 & 0 & 0 \\
1 & 0 & 1 & 0
\end{array}\right)\left(\begin{array}{c}
1 \\
-2 \\
-1 \\
2
\end{array}\right) \equiv\left(\begin{array}{l}
0 \\
0 \\
0 \\
0
\end{array}\right) \text {. }
$$


So, with respect to the ordered $\mathbb{F}_{2}$-basis $\left\{\partial_{1}^{G}, \partial_{B}^{G}, \partial_{A}^{G}, \partial_{G}^{G}\right\}$ of $\beta^{\times}(G)$, we have $\overline{\operatorname{torn}}_{G}^{\{p\}}\left(2 e_{G}^{G}\right)=(0,0,0,1)$ and $\overline{\operatorname{torn}}_{G}^{\{2\}}\left(2 e_{G}^{G}\right)=(0,0,0,0)$. Passing from additive to multiplicative notation, $(0,0,0,1)=1-2 e_{G}^{G}$ and $(0,0,0,0)=1$. We have obtained the asserted equation for $\overline{\operatorname{torn}}_{G}^{\pi}\left(2 e_{G}^{G}\right)$.

\begin{tabular}{|c|c|c|c|c|c|}
\hline \multicolumn{2}{|c|}{$\begin{array}{c}G=D_{2 p} \\
t_{I}^{\{p\}}[G / U]\end{array}$} & $\begin{array}{l}U \\
1 \\
\end{array}$ & $B$ & $A$ & $G$ \\
\hline \multirow[t]{4}{*}{$I$} & 1 & 0 & 0 & 0 & 0 \\
\hline & $B$ & 0 & 0 & 0 & 0 \\
\hline & $A$ & 2 & 1 & 0 & 0 \\
\hline & $G$ & 1 & 1 & 0 & 0 \\
\hline
\end{tabular}

\begin{tabular}{|c|c|c|c|c|}
\hline $\begin{array}{c}G=D_{2 p} \\
t_{I}^{\{2\}}[G / U]\end{array}$ & 1 & $B$ & $A$ & $G$ \\
\hline 1 & 0 & 0 & 0 & 0 \\
\hline$B$ & $p$ & $p-1$ & 1 & 0 \\
\hline$A$ & 0 & 0 & 0 & 0 \\
\hline$G$ & 1 & 0 & 1 & 0 \\
\hline
\end{tabular}

The set $\left\{\chi_{0}, \chi_{1}, \chi_{2}\right\}$ is a set of representatives for the Galois conjugacy classes of absolutely irreducible $\mathbb{R} G$-modules. So $\left\{\operatorname{ori}_{G}\left(\chi_{0}\right)\right.$, ori ${ }_{G}\left(\chi_{1}\right)$, ori $\left.{ }_{G}\left(\chi_{2}\right)\right\}$ is an $\mathbb{F}_{2}$-basis for $O_{\mathbb{R}}(G)$. Hence,

$$
\operatorname{zom}_{G}^{\alpha}\left(2 e_{G}^{G}\right)=z_{0}^{\pi} \operatorname{ori}_{G}\left(\chi_{0}\right)+z_{1}^{\pi} \operatorname{ori}_{G}\left(\chi_{1}\right)+z_{2}^{\pi} \operatorname{ori}_{G}\left(\chi_{2}\right)
$$

for some $z_{0}^{\pi}, z_{1}^{\pi}, z_{2}^{\pi} \in \mathbb{F}_{2}$. Since $\chi_{0}$ and $\chi_{1}$ are linear $\mathbb{R} G$-characters, we can use Proposition 9.2 to determine $z_{0}^{\pi}=z_{\chi_{0}}^{\pi}\left(2 e_{G}^{G}\right)$ and $z_{1}^{\pi}=z_{\chi_{1}}^{\pi}\left(2 e_{G}^{G}\right)$. The next pair of tables shows the values of $z_{\chi}^{\pi}[G / U]$ in the cases where $\chi \in\left\{\chi_{0}, \chi_{1}\right\}$ and $\pi \in\{\{p\},\{2\}\}$. Modulo 2 again,

$$
\left(\begin{array}{llll}
1 & 1 & 0 & 0 \\
1 & 0 & 0 & 0
\end{array}\right)\left(\begin{array}{c}
1 \\
-2 \\
-1 \\
2
\end{array}\right) \equiv\left(\begin{array}{l}
1 \\
1
\end{array}\right), \quad\left(\begin{array}{llll}
1 & 0 & 1 & 0 \\
1 & 0 & 1 & 0
\end{array}\right)\left(\begin{array}{c}
1 \\
-2 \\
-1 \\
2
\end{array}\right) \equiv\left(\begin{array}{l}
0 \\
0
\end{array}\right)
$$

So $z_{0}^{\{p\}}=z_{1}^{\{p\}}=1$ and $z_{0}^{\{2\}}=z_{1}^{\{2\}}=0$. Generally, $z_{0}^{\pi}$ is 0 or $z_{0}^{\{p\}}$ or $z_{0}^{\{2\}}$ or $z_{0}^{\{p\}}+z_{0}^{\{2\}}$, respectively, when $\pi \cap\{2, p\}$ is $\emptyset$ or $\{p\}$ or $\{2\}$ or $\{2, p\}$. A similar comment holds

\begin{tabular}{|c|c|c|c|c|}
\hline $\begin{array}{c}G=D_{2 p} \\
z_{\chi}^{\{p\}}[G / U]\end{array}$ & 1 & $B$ & $A$ & $G$ \\
\hline$\chi_{0}$ & 1 & 1 & 0 & 0 \\
\hline$\chi_{1}$ & 1 & 0 & 0 & 0 \\
\hline
\end{tabular}
for $z_{1}^{\pi}$. So, if $p \in \pi$ then $z_{0}^{\pi}=z_{1}^{\pi}=1$, while if $p \notin \pi$ then $z_{0}^{\pi}=z_{1}^{\pi}=0$.

\begin{tabular}{|c|c|c|c|c|}
\hline $\begin{array}{c}G=D_{2 p} \\
z_{\chi}^{\{2\}}[G / U]\end{array}$ & $U$ & $B$ & $A$ & $G$ \\
\hline$\chi_{0}$ & 1 & 0 & 1 & 0 \\
\hline$\chi_{1}$ & 1 & 0 & 1 & 0 \\
\hline
\end{tabular}

To determine $z_{2}^{\pi}$, we restrict to the subgroup $B$. The two irreducible $\mathbb{R} B$ characters are $\psi_{0}=\operatorname{res}\left(\chi_{0}\right)$ and $\psi_{1}=\operatorname{res}\left(\chi_{1}\right)$. Since $\psi_{0}$ and $\psi_{1}$ are absolutely irreducible and stable under Galois conjugation, the set $\left\{\operatorname{ori}_{B}\left(\psi_{0}\right), \operatorname{ori}_{B}\left(\psi_{1}\right)\right\}$ is an $\mathbb{F}_{2}$-basis for $O_{\mathbb{R}}(B)$. Since $\operatorname{res}\left(\chi_{2}\right)=\psi_{0}+\psi_{1}$, we have

$$
0=\operatorname{zom}_{B}^{\alpha}\left(\operatorname{res}_{B, G}\left(2 e_{G}^{G}\right)\right)=\operatorname{res}_{B, G}\left(\operatorname{zom}_{G}^{\alpha}\left(2 e_{G}^{G}\right)\right)=\left(z_{0}^{\pi}+z_{2}^{\pi}\right) \operatorname{ori}_{B}\left(\psi_{0}\right)+\left(z_{1}^{\pi}+z_{2}^{\pi}\right) \operatorname{ori}_{B}\left(\psi_{1}\right) .
$$

Therefore, if $p \in \pi$, then $z_{0}^{\pi}=z_{1}^{\pi}=z_{2}^{\pi}=1$ and $\operatorname{zom}_{G}^{\pi}\left(2 e_{G}^{G}\right)=\operatorname{ori}_{G}\left(\chi_{0}+\chi_{1}+\chi_{2}\right)$, while if $p \notin \pi$, then $z_{0}^{\pi}=z_{1}^{\pi}=z_{2}^{\pi}=0$ and $\operatorname{zom}_{G}^{\pi}\left(2 e_{G}^{G}\right)=0$. 
Let us mention an alternative to part of the latest proof. By direct calculation, it is easy to show that, under the hypothesis on $G$, the map $\underline{\operatorname{die}}_{G}: O_{\mathbb{R}}(G) \rightarrow B^{\times}(G)$ is an isomorphism. We have $\underline{\operatorname{die}}\left(\operatorname{ori}_{G}\left(\chi_{0}+\chi_{1}+\chi_{2}\right)\right)=\underline{\operatorname{die}}\left(\chi_{0}+\chi_{1}+\chi_{2}\right)=1-2 e_{G}^{G}$. So the equations for $\overline{\operatorname{torn}}_{G}^{\pi}\left(2 e_{G}^{\bar{G}}\right)$ and $\operatorname{zom}_{G}^{\alpha}\left(2 e_{G}^{G}\right)$ are equivalent to each other.

The methods used in the latest example-by restricting to smaller subgroups or, alternatively, by praying for $\underline{\operatorname{die}}_{G}$ to be injective-do not always enable us to determine the $\chi$ th coordinate $\left[\chi @ \operatorname{zom}_{G}^{\alpha}(\kappa)\right]$ for nonlinear $\chi$. Neither of those methods are applicable, for instance, in the case considered in Section 5, where $G=$ $S \ltimes C$ and $\chi$ is faithful. Indeed, in that particular case, the most practical method we have for calculating $\left[\chi @ \operatorname{zom}_{G}^{\alpha}(\kappa)\right]$ is by applying Corollary 6.9.

Corollary 9.7. Let $\pi^{\prime}$ be a set of primes such that $\pi^{\prime} \neq \pi$. Then $\overline{\operatorname{torn}}^{\pi^{\prime}} \neq \overline{\operatorname{torn}}^{\pi}$. Let $\alpha^{\prime}$ be an automorphism of $\mathbb{C}$ with Kummer symbol $\pi^{\prime}$. Then $\mathrm{zom}^{\alpha^{\prime}} \neq \mathrm{zom}^{\alpha}$.

Proof. Understanding that $D_{4}=V_{4}$, Examples 9.3 and 9.6 show that $\overline{\operatorname{torn}}_{D_{2 p}}^{\pi}$ is nonzero if and only if $p \in \pi$. The rider follows because $\overline{\text { torn }}^{\pi}$ factors through zom ${ }^{\alpha}$.

\section{FACTORIZING THROUGH THE REPRESENTATION FUNCTOR}

Does $\overline{\operatorname{torn}}^{\pi}$ factor through the morphism $\overline{\text { die }}: A_{\mathbb{R}} \rightarrow B^{\times}$? The question demands an answer because the morphisms $\overline{\exp }=\overline{\operatorname{die}} \circ$ lin $=\overline{\text { die }} \circ \overline{\text { in }}$ and $\overline{\text { torn }}^{\pi}=$ $\overline{\text { die }} \circ \mathrm{zom}^{\alpha}$ are, so to speak, siblings. Their kinship will become more clear in [4], especially in view of the uniqueness theorems in [3, Section 5] and also in view the discussion of deflation in [3, Appendix]. So, a priori, a factorization of $\overline{\operatorname{torn}}^{\pi}$ through die might seem plausible. Moreover, if such a factorization could be described explicitly, then it might seem likely that all the hard work in the proof of Theorem 2.3 could be dispensed with.

A more careful phrasing of the question is: given maps $\widetilde{z o m}_{G}^{\alpha}: K(G) \rightarrow$ $A_{\mathbb{R}}(G)$ such that $\operatorname{zom}_{G}^{\alpha}=\overline{\operatorname{die}}_{G} \circ \widetilde{z o m}_{G}^{\alpha}$, then which of the five elemental maps can commute with $\widetilde{z o m}_{G}^{\alpha}$, and for which classes of finite groups can those commutativity properties hold? Since $\overline{\operatorname{die}}_{G}$ commutes with deflation while $\overline{\operatorname{torn}}_{G}^{\pi}$ does not, $\widetilde{z o m}_{G}^{\pi}$ cannot commute with deflation. Since $K(C)=0$ for all cyclic groups $C$ and since an element of $A_{\mathbb{R}}(G)$ is zero when its restriction to all cyclic subgroups is zero, $\widetilde{z o m}_{G}^{\pi}$ cannot commute with restriction.

Theorem 10.1 implies that, for finite abelian groups, $\widetilde{z o m}_{G}^{\alpha}$ can commute with isogation, induction, and inflation. Actually, the main significance of Theorem 10.1 is that it provides an explicit formula for $\operatorname{zom}_{G}^{\alpha}$, albeit only in a very special case. However, Remark 10.4 tells us that, for finite 2-groups, $\widetilde{z o m}^{\alpha}$ cannot commute with isogation. Ultimately, this is a completely negative resolution of the matter because, to abandon commutativity with isogation would be to abandon transport of structure and group isomorphism invariance; one might as well abandon group theory entirely.

Theorem 10.1. Let $\operatorname{lin}_{G}^{\pi}$ be the restriction to $K(G)$ of the map $B(G) \rightarrow A_{\mathbb{R}}(G)$ given by

$$
[G / U] \mapsto[\mathbb{R} G / U] \log _{\pi}|G: U|
$$


for $U \leq G$. Then the maps $\operatorname{lin}_{G}^{\pi}$ commute with isogation, induction and inflation. Furthermore, if all the absolutely irreducible $\mathbb{R} G$-characters are linear, then $\operatorname{zom}_{G}^{\alpha}=$ ori $_{G o} \operatorname{lin}_{G}^{\pi}$ and $\overline{\operatorname{torn}}_{G}^{\pi}=\overline{\operatorname{die}}_{G o} l i n_{G}^{\pi}$.

To prove the theorem, we shall need the next two results. the first being a classification of the induction morphisms $\mathbb{C} B \rightarrow \mathbb{C} A_{\mathbb{C}}$. Let us define an elementgroup symbol to be a formal function $v$ such that, given any element $g$ of any finite group $I$, then $v(g, I) \in \mathbb{C}$ and, for any group isomorphism $\phi$ with domain $I$, we have $v(\phi(g), \phi(I))=v(g, I)$. When $I \leq G$ and $g \in G-I$, we understand that $v(g, I)=0$.

Proposition 10.2. There is a bijective correspondence between the induction morphisms $v: \mathbb{C} B \rightarrow \mathbb{C} A_{\mathbb{C}}$ and the element-group symbols $v$ such that $v \leftrightarrow v$ provided, for all $I \leq G \ni g$, the value of $v_{G}\left(e_{I}^{G}\right)$ at $g$ is

$$
v_{G}\left(e_{I}^{G}\right)(g)=\sum_{x N_{G}(I) \subseteq G} v\left({ }^{x} g, I\right) .
$$

Proof. Let $v$ be an element-group symbol, and let $v_{G}$ be the map $\mathbb{C} B(G) \rightarrow$ $\mathbb{C} A_{\mathbb{C}}(G)$ as specified by the formula. Obviously, the maps $v_{G}$ commute with isogation. Recall that ind $\mathrm{in}_{G, H}\left(e_{J}^{H}\right)=\left|N_{G}(J): N_{H}(J)\right| e_{J}^{G}$ for $J \leq H \leq G$. Regarding the elements ind $\operatorname{in}_{G, H}\left(v_{H}\left(e_{J}^{H}\right)\right)$ and $v_{G}\left(\operatorname{ind}_{G, H}\left(e_{J}^{H}\right)\right)$ as $G$-invariant functions $G \rightarrow \mathbb{C}$, and evaluating at an element $g \in G$, we have

$$
\begin{aligned}
\operatorname{ind}_{G, H}\left(v_{H}\left(e_{J}^{H}\right)\right)(g) & =\frac{1}{|H| \cdot\left|N_{H}(J)\right|} \sum_{x \in G, y \in H} v\left({ }^{y x} g, J\right) \\
& =\frac{1}{\left|N_{H}(J)\right|} \sum_{x \in G} v\left({ }^{x} g, J\right)=v_{G}\left(\operatorname{ind}_{G, H}\left(e_{J}^{H}\right)\right)(g) .
\end{aligned}
$$

So the maps $v_{G}$ commute with induction. Thus, any element-group symbol $v$ determines a corresponding induction morphism $v$. Conversely, any induction morphism $v$ determines a corresponding element-group symbol $v$ by $v(g, I)=$ $v_{I}\left(e_{I}^{I}\right)(g)$, whereupon

$$
v_{G}\left(e_{I}^{G}\right)(g)=\frac{1}{\left|N_{G}(I): I\right|} \operatorname{ind}_{G, I}\left(v_{I}\left(e_{I}^{I}\right)\right)(g)=\sum_{x N_{G}(I) \subseteq G} v\left({ }^{x} g, I\right) .
$$

Evidently, the correspondences $v \mapsto v$ and $v \mapsto v$ are mutually inverse.

Let $v^{\pi}: \mathbb{C} B \rightarrow \mathbb{C} A_{\mathbb{C}}$ be the induction morphism whose corresponding elementgroup symbol $v^{\pi}$ is given by

$$
v^{\pi}(g, I)=\sum_{U: g \in U \leq I} \mu(U, I) \log _{\pi}|I: U|
$$

Note that, if $I$ is a noncyclic subgroup of $G$, then

$$
v^{\pi}(g, I)=\sum_{U: g \in U \leq I} \mu(U, I) \log _{\pi}|G: U|
$$

because $\log _{\pi}|G: U|=\log _{\pi}|G: I|+\log _{\pi}|I: U|$ and $\sum_{U} \mu(U, I)=0$. 
Lemma 10.3. The map $v_{G}^{\pi}$ restricts to the map $\operatorname{lin}_{G}^{\pi}: K(G) \rightarrow A_{\mathbb{R}}(G)$.

Proof. The $\mathbb{C}$-vector space $\mathbb{C} K(G)$ has a basis consisting of the elements having the form $e_{I}^{G}$ where $I$ is a noncyclic subgroup of $G$. Linearly extending $\operatorname{lin}_{G}^{\pi}$ to a map $\mathbb{C} K(G) \rightarrow \mathbb{C} A_{\mathbb{C}}(G)$, it suffices to show that $\operatorname{lin}_{G}^{\pi}\left(e_{I}^{G}\right)=v_{G}^{\pi}\left(e_{I}^{G}\right)$ for all noncyclic $I$. The value of the permutation character $[\mathbb{R} G / U]$ at an element $g \in G$ is $[\mathbb{R} G / U](g)=\left|\left\{x \in G:{ }^{x} g \in U\right\}\right| /|U|$. Combining this with the above formula for $e_{I}^{G}$, an easy manipulation yields $\operatorname{lin}_{G}^{\pi}\left(e_{I}^{G}\right)(g)=v_{G}^{\pi}\left(e_{I}^{G}\right)(g)$.

Let us now prove Theorem 10.1. It is obvious that the maps $\operatorname{lin}_{G}^{\pi}$ commute with inflation and isogation. By the latest two results, $\operatorname{lin}_{G}^{\pi}$ also commutes with induction. Now let $\chi$ be a linear $\mathbb{R} G$-character. As we noted in the proof of Proposition 9.2, $\chi$ occurs in $\mathbb{R} G / U$ if and only if $U \leq \operatorname{Ker}(\chi)$ and, in that case, $\chi$ occurs exactly once. So, by Proposition 9.2, $\left[\chi @ \operatorname{zom}_{G}^{\alpha}(\kappa)\right] \stackrel{\text { par }}{=}\left[\chi @ \operatorname{lin}_{G}^{\pi}(\kappa)\right]$. The rider to Theorem 10.1 now follows, and the proof of the theorem is complete.

The rider to the theorem can fail if we drop the hypothesis on $G$. In the next result, we shall show that the case $G=D_{8}$ is a counter-example. For the sake of a more general discussion in the next section, let us recall some material from Bouc [7, Section 6] concerning the non-abelian dihedral 2-groups in general.

Given an integer $n \geq 3$, then the dihedral group $D_{2^{n}}$ has generators $a$ and $s$ such that, writing $t=a s$, the relations are $a^{2^{n-1}}=s^{2}=t^{2}=1$. Letting integers $i$ and $j$ run over the ranges $0 \leq i \leq n-1$ and $1 \leq j \leq n-1$, then the subgroups

$$
A_{i}=\left\langle a^{2^{n-1-i}}\right\rangle \cong C_{2^{i}}, \quad S_{j}=\left\langle A_{j-1}, s\right\rangle \cong D_{2^{j}}, \quad T_{j}=\left\langle A_{j-1}, t\right\rangle \cong D_{2^{j}}
$$

comprise a set of representatives of the conjugacy classes of strict subgroups of $D_{2^{n}}$. The boundary element of $K\left(D_{2^{n}}\right)$ is defined to be

$$
\kappa_{n}=\left[D_{2^{n}} / S_{2}\right]-\left[D_{2^{n}} / T_{2}\right]-\left[D_{2^{n}} / S_{1}\right]+\left[D_{2^{n}} / T_{1}\right] .
$$

(We point out that, for a given group $G$ isomorphic to $D_{2^{n}}$, the boundary element of $K(G)$ is well defined only up to a \pm sign, since an outer automorphism of $D_{2^{n}}$ interchanges the conjugacy classes $S_{j}$ and $T_{j}$.) Gluck's Idempotent Formula gives a quick proof that $\kappa_{n}$ does indeed belong to $K\left(D_{2^{n}}\right)$. Writing $D=D_{2^{n}}$, it is easy to show that $4 e_{S_{2}}^{D}=[D / 1]-\left[D / A_{1}\right]-2\left[D / S_{1}\right]+2\left[D / S_{2}\right]$ and similarly for $e_{S_{2}}^{D}$. Hence

$$
\kappa_{n}=2\left(e_{S_{2}}^{D}-e_{T_{2}}^{D}\right) \text {. }
$$

Since $S_{2} \cong T_{2} \cong V_{4}$, which is noncyclic, we deduce that $\kappa_{n} \in K(D)$. A different proof of this fact is given in [7, 6.10].

Remark 10.4. Suppose that $G=D_{8}$. Let $\phi$ be an outer automorphism of $G$. Then there does not exist a map $\widetilde{\operatorname{zom}}_{G}^{\{2\}}$ such that $\overline{\operatorname{torn}}_{G}^{\{2\}}=\overline{\operatorname{die}}_{G \circ} \widetilde{\operatorname{zom}}_{G}^{\{2\}}$ and $\widetilde{\operatorname{zom}_{G}}\{$ commutes with iso ${ }_{G}^{\phi}$. In particular, there does not exist an isogation morphism $\widetilde{\mathrm{zom}}^{\{2\}}$ such that $\overline{\operatorname{torn}}^{\{2\}}=\overline{\mathrm{die}}_{\mathrm{o}} \widetilde{\mathrm{zom}}^{\{2\}}$.

Proof. Let $\widetilde{\operatorname{zom}}_{G}^{\{2\}}$ be a $\mathbb{Z}$-linear map $K(G) \rightarrow A_{\mathbb{R}}(G)$ such that $\overline{\operatorname{torn}}_{G}^{\{2\}}=$ $\overline{\operatorname{die}}_{G \circ} \widetilde{z o m}_{G}^{\{2\}}$. Let $\chi_{S}$ and $\chi_{T}$ be the linear $\mathbb{R} G$-characters with kernels $S_{2}$ and 
$T_{2}$, respectively. Let $\chi$ be the unique faithful irreducible $\mathbb{R} G$-character. A straightforward application of the method illustrated in Example 9.6 yields

$$
\overline{\operatorname{torn}}_{G}^{\{2\}}\left(\kappa_{3}\right)=\partial_{P}+\partial_{Q}=\overline{\operatorname{die}}\left(\chi_{S}+\chi_{T}+\chi\right) .
$$

(These are special cases of two equalities proved in the next section.) But $\overline{\operatorname{die}}_{G}$ is an isomorphism by Theorem 2.1 , so the $\chi$-coordinate $\left\langle\chi \mid \widetilde{\operatorname{zom}}_{G}^{\{2\}}\left(\kappa_{3}\right)\right\rangle$ is an odd integer, perforce nonzero. For an outer automorphism $\phi$ of $G$, the action of iso ${ }_{G}^{\phi}$ on $A_{\mathbb{R}}(G)$ fixes $\chi$ and interchanges $\chi_{S}$ and $\chi_{T}$, so

$$
\left\langle\chi \mid \operatorname{iso}_{G}^{\phi}\left(\widetilde{\operatorname{zom}}_{G}^{\{2\}}\left(\kappa_{3}\right)\right)\right\rangle=\left\langle\chi \mid \widetilde{\operatorname{zom}}_{G}^{\{2\}}\left(\kappa_{3}\right)\right\rangle \neq 0 .
$$

Since the action of $\phi$ interchanges the conjugacy classes of $S_{j}$ and $T_{j}$, we have $\operatorname{iso}_{G}^{\phi}\left(\kappa_{3}\right)=-\kappa_{3}$ and

$$
\left\langle\chi \mid \widetilde{\operatorname{zom}}_{G}^{\{2\}}\left(\operatorname{iso}_{G}^{\phi}\left(\kappa_{3}\right)\right)\right\rangle=-\left\langle\chi \mid \widetilde{\operatorname{zom}}_{G}^{\{2\}}\left(\kappa_{3}\right)\right\rangle .
$$

Therefore, iso ${ }_{G}^{\phi}\left(\widetilde{\mathrm{zom}}_{G}^{\{2\}}\left(\kappa_{3}\right)\right) \neq{\widetilde{\mathrm{zom}_{G}}}_{G}^{\{2\}}\left(\operatorname{iso}_{G}^{\phi}\left(\kappa_{3}\right)\right)$.

The remark also shows that the zombie modules for $\kappa_{3}$ are not well defined up to isomorphism. In the next section, we shall show that, for $n \geq 4$, even in the modulo 2 reduction $\mathbb{F}_{2} A_{\mathbb{R}}\left(D_{2^{n}}\right)$, the images of the zombie modules for $\kappa_{n}$ are not well defined.

\section{WHY THE ORIENTATION FUNCTOR?}

We shall calculate the images of the boundary elements $\kappa_{n} \in K\left(D_{2^{n}}\right)$ under the reduced Tornehave map $\overline{\operatorname{torn}}_{D_{2^{n}}}^{\pi}$ and the zombie map zom ${ }_{D_{2^{n}}}^{\alpha}$. Generally, any serious study of the morphisms $\overline{\operatorname{torn}}^{\pi}$ and zom $^{\alpha}$ must surely require some guidance from concrete data, and the concrete examples $\kappa=\kappa_{n}$ are surely the first examples to consider, since Bouc [7, 6.12] tells us that, for 2-groups, the biset functor $K$ is generated by the elements $\kappa_{n}$ for $n \geq 3$.

That data will be of use to us in the other main purpose of this section, which is to address the question: Why $O_{\mathbb{R}}$ ? This article arose from a search for a refined sense in which a zombie module $W$ of an element $\kappa \in K(G)$ is well defined. Proposition 11.1 and Corollary 11.5 both reveal, in different ways, that $W$ is very far from being well defined up to isomorphism. But that does not squash the proposal that perhaps $W$ could become well defined, modulo some refined equivalence, if we were to impose some suitable constraints on the choices made in the construction of $W$. Thus the problem, stated more precisely, is to find an inflaky subfunctor $J \leq$ $\operatorname{Ker}(\overline{\mathrm{die}})$ such that $\overline{\text { torn }}^{\pi}$ factors through the inflaky morphism $A_{\mathbb{R}} / J \rightarrow B^{\times}$induced

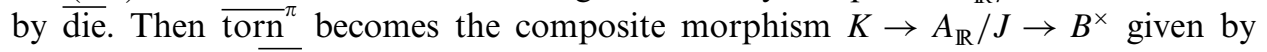
$\kappa \mapsto[W]+J \mapsto \overline{\operatorname{die}}_{G}[W]=\operatorname{torn}_{G}^{\pi}(\kappa)$. The element $[W]+J \in A_{\mathbb{R}}(G) / J(G)$ must be well defined, but it is conceivable that this could be ensured by imposing suitable constraints on the construction of $W$.

Of course, one solution to the problem is to put $J=\operatorname{Ker}(\overline{\operatorname{die}})$. Our more refined solution has been to put $J=A_{\mathbb{R}} \cap I_{\mathbb{C}}$, the factorization becoming $\overline{\operatorname{torn}}^{\pi}=$ 
die $_{\text {ozom }}{ }^{\alpha}$. The quotient functor $O_{\mathbb{R}}=A_{\mathbb{R}} /\left(A_{\mathbb{R}} \cap I_{\mathbb{C}}\right)$ has the advantage that, as we saw in Remark 4.1, the coordinate modules $O_{\mathbb{R}}(G)$ can be described quite explicitly. Nevertheless, one can still ask as to whether there exists an even smaller subfunctor $J$ that would yield an even more refined factorization of torn ${ }^{\pi}$. Below, we shall not actually prove the negative answer, but we shall give some compelling reasons for putting $J=A_{\mathbb{R}} \cap I_{\mathbb{C}}$.

Remark 10.4 says that, even as an isogation morphism, $\overline{\operatorname{torn}}^{\pi}$ does not factorize through $\overline{\text { die, }}$, so we cannot put $J=0$. The next result tells us that, if we do not impose any constraints on the construction of $W$, then we must have $J \geq 2 A_{\mathbb{R}}$, since otherwise the element $[W]+J \in A_{\mathbb{R}} / J$ would not be well defined.

Proposition 11.1. Let $\kappa \in K(G)$, let $W$ be a zombie $\mathbb{R} G$-module for $\kappa$, and let $M$ be any $\mathbb{R} G$-module. Then $W \oplus M \oplus M$ is a zombie $\mathbb{R} G$-module for $\kappa$.

Proof. Let $X, Y, \imath, \theta, \theta_{\mathbb{R}}$ be as before, with $\kappa=[X]-[Y]$ and with $W$ as the $(-1)$ eigenspace of $\theta_{\mathbb{R}}$. Let $Z$ be a $G$-set such that $M$ is isomorphic to a direct summand of $\mathbb{R} Z$. We can write $\mathbb{R} Z=M \oplus N$ as a direct sum of two mutually orthogonal $\mathbb{R} G$-modules, the inner product on $\mathbb{R} Z$ being such that $Z$ is an orthonormal basis. Let $Z^{\prime}$ be a $G$-set isomorphic to $Z$, and let $\phi: Z \rightarrow Z^{\prime}$ be an isomorphism. The $\mathbb{R}$ linear extension $\phi: \mathbb{R} Z \rightarrow \mathbb{R} Z^{\prime}$ is a $G$-invariant isometry. Letting $X_{+}=X \sqcup Z \sqcup Z^{\prime}$ and $Y_{+}=Y \sqcup Z \sqcup Z^{\prime}$, then $\kappa=\left[X_{+}\right]-\left[Y_{+}\right]$.

As direct sums of mutually orthogonal $\mathbb{R} G$-modules,

$$
\mathbb{R} X_{+}=\mathbb{R} X \oplus M \oplus M^{\prime} \oplus N_{+}, \quad \mathbb{R} Y_{+}=\mathbb{R} Y \oplus M \oplus M^{\prime} \oplus N_{+}
$$

where $M^{\prime}=\phi(M)$ and $N_{+}=N \oplus \phi(N)$. Consider variables

$$
\xi \in \mathbb{R} X, \quad \eta \in \mathbb{R} Y, \quad \mu \in M, \quad m^{\prime} \in M^{\prime}, \quad v \in N_{+} .
$$

Any element of $\mathbb{R} X$ can be written uniquely in the form $\xi+\mu+\mu^{\prime}+v$. Any element of $\mathbb{R} Y$ can be written uniquely in the form $\eta+\mu+\mu^{\prime}+v$. Let $c$ and $s$ be real numbers such that $c^{2}+s^{2}=1$. Let $l_{+}: \mathbb{R} Y_{+} \rightarrow \mathbb{R} X_{+}$be the map of $\mathbb{R} G$-modules such that

$$
l_{+}(\eta)=\imath(\eta), \quad \imath(\mu)=c \mu+s \phi(\mu), \quad \imath\left(\mu^{\prime}\right)=-s \phi^{-1}\left(\mu^{\prime}\right)+c \mu^{\prime}, \quad l(v)=v .
$$

Thus $l_{+}$is the extension of $l$ such that $l_{+}$acts as the identity operator on $N_{+}$while $l_{+}$acts on each Euclidian plane $\mathbb{R} \mu \oplus \mathbb{R} \phi(\mu)$ as the rotation through angle $\vartheta$, where $c=\cos (\vartheta)$ and $s=\sin (\vartheta)$. Since $l_{+}^{-1}$ acts on those Euclidian planes as the rotation though angle $-\vartheta$, we have

$$
l_{+}^{-1}\left(\xi+\mu+\mu^{\prime}+v\right)=l^{-1}(\xi)+\left(c \mu+s \phi^{-1}\left(\mu^{\prime}\right)\right)+\left(-s \phi(u)+c \mu^{\prime}\right)+v
$$

(the extra brackets displaying the expression as a sum of four vectors in $\mathbb{R} X, M, M^{\prime}$, $N_{+}$, respectively). Let $\theta_{+}=l_{+\circ} \alpha_{\circ} l_{+}^{-1} \alpha^{-1}$. Using the fact that $\alpha$ commutes with $\phi$, a straightforward calculation yields

$$
\begin{aligned}
\theta_{+}\left(\xi+\mu+\mu^{\prime}+v\right)= & \theta(\xi)+(c \alpha(c)+s \alpha(s))\left(\mu+\mu^{\prime}\right) \\
& +(c \alpha(s)-\alpha(c) s) \phi^{-1}\left(\mu^{\prime}\right)+(\alpha(c) s-c \alpha(s)) \phi(\mu)+v
\end{aligned}
$$


Now let $p$ be the smallest prime in $\pi$, and put $c=1 / \sqrt{p}$. Then $s= \pm \sqrt{(p-1) / p}$. Since $\alpha(\sqrt{p})=-\sqrt{p}$ and $\alpha(\sqrt{p-1})=\sqrt{p-1}$, we have $\alpha(c)=-c$ and $\alpha(s)=-s$. Hence

$$
\theta_{+}\left(\xi+\mu+\mu^{\prime}+v\right)=\theta(\xi)-\mu-\mu^{\prime}+v
$$

Since the $\mathbb{C}$-linear operator $\theta_{+}$already acts as an $\mathbb{R}$-linear operator on $M \oplus M^{\prime} \oplus$ $N_{+}$, we can deform $\theta_{+}$to an operator $\theta_{+, \mathbb{R}} \in O_{G}\left(\mathbb{R} X_{+}\right)$such that

$$
\theta_{+, \mathbb{R}}\left(\xi+\mu+\mu^{\prime}+v\right)=\theta_{\mathbb{R}}(\xi)-\mu-\mu^{\prime}+v .
$$

The (-1)-eigenspace $\theta_{+, \mathbb{R}}$ is $W \oplus M \oplus M^{\prime}$.

Before continuing with our justification for putting $J=A_{\mathbb{R}} \cap I_{\mathbb{C}}$, we need to make a study of the non-abelian dihedral 2-groups. For the rest of this article, we let $D=D_{2^{n}}$ with $n \geq 3$, and we employ the notation introduced in the previous section. We shall assume that $2 \in \pi$, since otherwise $\overline{\operatorname{torn}}_{D}^{\pi}$ and $\operatorname{zom}_{D}^{\alpha}$ would be the zero maps.

Example 11.2. For all $n \geq 3$, we have $\overline{\operatorname{torn}}_{D}^{\pi}\left(\kappa_{n}\right)=\partial_{S_{2}}^{D}+\partial_{T_{2}}^{D}+\partial_{S_{3}}^{D}+\partial_{T_{3}}^{D}+\cdots+$ $\partial_{S_{n-1}}^{D}+\partial_{T_{n-1}}^{D}$.

Proof. We are to show that, given $I \leq D$, then $\left[\operatorname{torn}_{D}^{\pi}\left(\kappa_{n}\right) @ I\right]=0$ if and only if $I=$ $S_{j}$ or $I=T_{j}$ with $2 \leq j \leq n-1$. We shall apply Remark 9.1. The integer $t_{A_{i}} \pi\left(\kappa_{n}\right)$ is even because $t_{A_{i}} \pi\left[D / S_{j}\right]=t_{A_{i}} \pi\left[D / T_{j}\right]$ for all $0 \leq i \leq n-1$ and all $1 \leq j \leq n-1$. So $\left[\overline{\operatorname{torn}}_{D}^{\pi}\left(\kappa_{n}\right) @ A_{j}\right]=0$. Similarly, $\left[\overline{\operatorname{torn}}_{D}^{\pi}\left(\kappa_{n}\right) @ D\right]=0$. In the notation of Remark 9.1, putting $I=S_{j}$ and $U \in\left\{S_{1}, T_{1}, S_{2}, T_{2}\right\}$, the integer partitions are easily seen to be

$$
\begin{aligned}
& \left|D: S_{1}\right|=2 \cdot 2^{j-1}+\left(2^{n-j-1}-1\right) \cdot 2^{j}, \quad\left|D: T_{1}\right|=2^{n-1-j} \cdot 2^{j}, \\
& \left|D: S_{2}\right|= \begin{cases}2 \cdot 4+\left(2^{n-3}-1\right) \cdot 8 & \text { if } j=1, \\
2 \cdot 2^{j-1}+\left(2^{n-1-j}-1\right) \cdot 2^{j-1} & \text { if } 2 \leq j \leq n-1,\end{cases} \\
& \left|D: T_{2}\right|= \begin{cases}2^{n-3} \cdot 2 & \text { if } j=1, \\
2^{n-1-j} \cdot 2^{j-1} & \text { if } 2 \leq j \leq n-1 .\end{cases}
\end{aligned}
$$

By the formula in Remark 9.1,

$$
\begin{gathered}
{\left[\operatorname{torn}_{D}^{\pi}\left[D / S_{1}\right] @ S_{j}\right] \stackrel{\text { par }}{=} \begin{cases}j & \text { if } 1 \leq j \leq n-2, \\
0 & \text { if } j=n-1,\end{cases} } \\
{\left[\operatorname{torn}_{D}^{\pi}\left[D / T_{1}\right] @ S_{j}\right] \stackrel{\text { par }}{=} \begin{cases}0 & \text { if } 1 \leq j \leq n-2, \\
n-1 & \text { if } j=n-1,\end{cases} } \\
{\left[\operatorname{torn}_{D}^{\pi}\left[D / S_{2}\right] @ S_{j}\right] \stackrel{\text { par }}{=} \begin{cases}1 & \text { if } j=1, \\
j-1 & \text { if } 2 \leq j \leq n-2, \\
0 & \text { if } j=n-1,\end{cases} }
\end{gathered}
$$




$$
\left[\operatorname{torn}_{D}^{\pi}\left[D / T_{2}\right] @ S_{j}\right] \stackrel{\text { par }}{=} \begin{cases}0 & \text { if } 1 \leq j \leq n-2, \\ 1 & \text { if } j=n-1 .\end{cases}
$$

The defining formula for $\kappa_{n}$ now yields $\left[\operatorname{torn}_{D}^{\pi}\left(\kappa_{n}\right) @ S_{j}\right] \stackrel{\text { par }}{=}\left\{\begin{array}{cc}0 & \text { if } j=1, \\ 1 & \text { if } 2 \leq j \leq n-1 .\end{array}\right.$

We shall calculate the element $\operatorname{zom}_{D}^{\alpha}\left(\kappa_{n}\right) \in O_{\mathbb{R}}(D)$. But first let us describe the orientation group $O_{\mathbb{R}}(D)$. Let $\chi_{D}, \chi_{A}, \chi_{S}, \chi_{T}$ be the linear $\mathbb{R} D$-characters with kernels $D, A, S_{n-1}, T_{n-1}$, respectively. Let $d=2^{n-1}$. Let $r$ be an integer running over the range $1 \leq r \leq d / 2-1$, and let $\chi_{r}$ be the 2-dimensional $\mathbb{R} D$-character whereby $s$ acts as a reflection and $a$ acts as a rotation through an angle of $\pm 2 \pi r / d$. The irreducible $\mathbb{R} D$-characters are $\chi_{D}, \chi_{A}, \chi_{S}, \chi_{T}$ and $\chi_{r}$. All of these characters are absolutely irreducible. Two characters having the form $\chi_{r}$ and $\chi_{r^{\prime}}$ are Galois conjugate if and only if $\log _{2} r=\log _{2} r^{\prime}$. So the characters $\chi_{D}, \chi_{A}, \chi_{S}, \chi_{T}$, and $\chi_{2^{k}}$ with $0 \leq k \leq n-$ 3 comprise a set of representatives of the Galois conjugacy classes of absolutely irreducible $\mathbb{R} D$-characters. Via Remark 4.1, we obtain the next result.

Example 11.3. The elements $\operatorname{ori}_{D}\left(\chi_{D}\right), \operatorname{ori}_{D}\left(\chi_{A}\right), \operatorname{ori}_{D}\left(\chi_{S}\right), \operatorname{ori}_{D}\left(\chi_{T}\right)$, and $\operatorname{ori}_{D}\left(\chi_{2^{k}}\right)$ with $0 \leq k \leq n-3$ comprise an $\mathbb{F}_{2}$-basis for $O_{\mathbb{R}}(D)$.

We shall express $\operatorname{zom}_{D}^{\pi}\left(\kappa_{n}\right)$ in coordinate form with respect to that basis. Given $I \leq D$, it is easy to show that the $I$-fixed subspace of the $\mathbb{R} G$-module affording $\chi_{S}+$ $\chi_{T}+\chi_{1}$ has odd dimension if and only if $I=S_{j}$ or $I=T_{j}$ with $2 \leq j \leq n-1$. This, together with Example 11.2, yields $\overline{\operatorname{die}}_{D}\left(\chi_{S}+\chi_{T}+\chi_{1}\right)=\overline{\operatorname{torn}}_{D}^{\pi}\left(\kappa_{n}\right)$. But $\overline{\operatorname{torn}}_{D}^{\pi}=$ $\underline{\text { die }}_{D \circ}$ zom $_{D}^{\alpha}$ and, by Theorem 2.1, $\underline{\text { die }}_{D}$ is injective. The next result follows.

Example 11.4. For all $n \geq 3$, we have $\operatorname{zom}_{D}^{\alpha}\left(\kappa_{n}\right)=\operatorname{ori}_{D}\left(\chi_{S}+\chi_{T}+\chi_{1}\right)$.

The faithful irreducible $\mathbb{R} D$-characters are the characters $\chi_{r}$ where $1 \leq r \leq$ $d / 2-1$ and $r$ is odd. There are $d / 4$ such characters and they comprise a single Galois conjugacy class. When $n \geq 4$, the integer $d / 4=2^{n-3}$ is even, so Example 11.4 has the following corollary.

Corollary 11.5. Suppose that $n \geq 4$, and let $W$ be a zombie $\mathbb{R} D$-module for $\kappa_{n}$. Then the faithful irreducible $\mathbb{R} D$-characters comprise a single Galois conjugacy class, the number of such characters is even, and the number of such characters occuring with odd multiplicity in $W$ is odd. In particular, there exists an automorphism $\gamma$ of $\mathbb{C}$ such that $[W]-\left[{ }^{\gamma} W\right] \notin 2 A_{\mathbb{R}}(D)$.

We can now complete our justification for putting $J=A_{\mathbb{R}} \cap I_{\mathbb{C}}$. Let us assume that the function $K(G) \ni \kappa \mapsto[W]+J \in A_{\mathbb{R}}(G) / J(G)$ is well defined, where $G$ is an arbitrary finite group and $W$ is any zombie module of $\kappa$. Above, we proved that the assumption implies the inequality $J \geq 2 A_{\mathbb{R}}$. Corollary 11.5 implies that, in fact, the inequality is strict, $J>A_{\mathbb{R}}$. The corollary also suggests (but does not imply) that $J \geq$ $S$, where $S$ is the biset subfunctor of $A_{\mathbb{R}}$ such that $S(G)$ is the $\mathbb{Z}$-span of those virtual $\mathbb{R} G$-characters which can be written as a difference $\chi-\chi^{\prime}$ of two Galois conjugate $\mathbb{C} G$-characters. We arrive at the inequality $J \geq 2 A_{\mathbb{R}}+S=A_{\mathbb{R}} \cap I_{\mathbb{C}}$. Theorem 2.3 tells us that, if we take $J$ to be the minimal solution to that inequality, namely, 
$J=A_{\mathbb{R}} \cap I_{\mathbb{C}}$, then $\overline{\operatorname{torn}}^{\pi}$ does indeed factorize through the morphism $A_{\mathbb{R}} / J \rightarrow B^{\times}$ induced by die.

\section{NO SYMMETRY-BREAKING}

It is worth making a further examination of the scenario of Corollary 11.5. As in the previous section, we consider the group $D=D_{2^{n}}$ but, for convenience of discussion, we now assume that $n \geq 4$. (The calculations are still valid for $n=3$, but parts of our commentary fail in that case.) We shall show that, in the construction of a zombie $\mathbb{R} D$-module module $W$ of $\kappa_{n}$, the isomorphism class of $W$ depends not only on the choice of isometry $\imath$ but also on the choice of automorphism $\alpha$, even when the Kummer symbol $\pi$ is fixed.

The calculations in this section lie at the heart of the work behind this article, because Proposition 12.1 served as a "no-go theorem," giving the clue that led to the hypothesis $\alpha(\mathbb{C} U) \cong \mathbb{C} U$ that is needed in Corollary 6.9. In fact, the "no go theorem" is quite subtle and, as we shall see, it is associated with an illusion of a symmetrybreaking paradox which needs to be dispelled along the way.

We must add to the notation of the previous section. By the definition of $\kappa_{n}$, we have $\kappa_{n}=[X]-[Y]$ where $X$ and $Y$ are the $D$-sets $X=D / T_{1} \sqcup D / S_{2}$ and $Y=$ $D / S_{1} \sqcup D / T_{2}$. As before, let $\imath: \mathbb{R} Y \rightarrow \mathbb{R} X$ be a $D$-invariant isometry, and let $\theta=$ $\imath \circ \alpha \circ l^{-1} \circ \alpha^{-1}$. Let $\theta_{\mathbb{R}}$ be an element of $O_{D}(\mathbb{R} X)$ such that $\theta$ and $\theta_{\mathbb{R}}$ belong to the same connected component of $O_{D}(\mathbb{C} X)$. We take $W$ to be the $(-1)$-eigenspace of $\theta_{\mathbb{R}}$. Let $d=2^{n-1}$. A straightforward application of character theory yields

$$
[\mathbb{R} X]=[\mathbb{R} Y]=2 \chi_{D}+\chi_{S}+\chi_{T}+2 \sum_{q} \chi_{q}+\sum_{r \in \mathscr{R}} \chi_{r},
$$

where $q$ runs over the even integers in the range $2 \leq q \leq d / 2-2$ and $\mathscr{R}$ is the set of odd integers $r$ in the range $1 \leq r \leq d / 2-1$. The term $\sum_{r \in \mathscr{R}} \chi_{r}$ is the sum of the faithful irreducible $\mathbb{R} D$-characters $\chi_{r}$. Corollary 11.5 now implies that an odd number of those characters occur in the $\mathbb{R} D$-submodule $W \leq \mathbb{R} X$.

Given an even number of indistinguishable objects, there does not exist a deterministic rule for selecting an odd number of them. Since $n \geq 4$, there are an even number of faithful irreducible $\mathbb{R} D$-characters $\chi_{r}$, and they comprise Galois conjugacy class, so how is it that an odd number of them appears in $W$ ? Of course, the construction of $W$ involved arbitrary choices of $X, Y, \alpha, l, \theta_{\mathbb{R}}$. But let us look at those choices carefully. We have already fixed $X$ and $Y$. The parameter $\alpha$ can be regarded as given, and we have registered it as a given variable in our notation zom $^{\alpha}$. In the course of the argument below, we shall see that, when the choices of $X$, $Y, \alpha, l$ have been made, there is no freedom of choice for $\theta_{\mathbb{R}}$. So, having fixed $X, Y$, and $\alpha$, the selection of those faithful $\chi_{r}$ that are to appear in $W$ must be determined by the choice of $l$.

But that is not enough to satisfy the question. Let $e_{r}$ be the primitive idempotent of $Z(\mathbb{R} D)$ associated with $\chi_{r}$. The $\chi_{r}$-components of $\mathbb{R} X$ and $\mathbb{R} Y$ are the simple submodules $e_{r} \mathbb{R} X$ and $e_{r} \mathbb{R} Y$, which both have character $\chi_{r}$. For each $r \in \mathscr{R}$, there are precisely two choices for the restriction of $\imath$ to an isometry $l_{r}: e_{r} \mathbb{R} Y \rightarrow$ $e_{r} \mathbb{R} Y$. As $r$ varies, the choices of the isometries $l_{r}$ can be made independently. Suppose there are an even number of indistinguishable objects. An imp arbitrarily 
colours each of the objects black or white. Then there still does not exist a deterministic rule for selecting an odd number of the objects. Indeed, were the imp to unhelpfully colour all of them black, we would be reduced to the same situation as before. So we appear to have a paradox: having fixed $X, Y$, and $\alpha$, then the selection of those $r \in \mathscr{R}$ such that $\chi_{r}$ is to appear in $W$ must be determined by the independent arbitrary choices of the isometries $l_{r}$. The two possible values for each $l_{r}$ can be labeled black and white. Thus, an arbitrary black-white colouring of the even-sized set $\mathscr{R}$ is to determine an odd-sized subset!

To spoil the tension, let us resolve the paradox immediately. The characters $\chi_{r}$ are, indeed, symmetrically indistinguishable in the sense of Galois conjugacy. For each $r \in \mathscr{R}$, there are, indeed, two possible values for the isometry $l_{r}$. However, there is no Galois-invariant rule for assigning the colours black and white to those two values.

Still, we surely do now have a reason for wishing to bring the scenario into the light with some explicit formulas and concrete coordinates. Our purpose is to find out just how the choices of $\imath$ and $\alpha$ determine those indices $r \in \mathscr{R}$ such that $e_{r} \mathbb{R} X$ is a summand of the submodule $W \leq \mathbb{R} X$. The calculations-which also hold for $n=3$-will yield an alternative proof of Corollary 11.5.

Let $T=T_{1}$ and $S=S_{1}$. Since $\chi_{r}$ occurs in $\mathbb{R} D / T$ and in $\mathbb{R} D / S$, we have $e_{r} \mathbb{R} X=e_{r} \mathbb{R} D / T$ and $e_{r} \mathbb{R} Y=e_{r} \mathbb{R} D / S$. We claim that $e_{r} \mathbb{R} X$ has orthonormal basis $\left\{\xi_{r}^{1}, \xi_{r}^{2}\right\}$ where $\xi_{r}^{1}=2^{n / 2-1} e_{r} T$ and $\xi_{r}^{2}=a^{d / 4} \xi_{r}^{1}$. Writing $\operatorname{cs}(\lambda)=\cos (2 \pi \lambda)$ and $\operatorname{sn}(\lambda)=$ $\sin (2 \pi \lambda)$ for $\lambda \in \mathbb{R}$,

$$
e_{r}=\frac{\chi_{r}(1)}{|D|} \sum_{g \in D} \chi_{r}\left(g^{-1}\right) g=2^{2-n} \sum_{k=0}^{d-1} \operatorname{cs}(r k / d) a^{k}, \quad \xi_{r}^{1}=2^{1-n / 2} \sum_{k=0}^{d-1} \operatorname{cs}(r k / d) a^{k} T .
$$

By the definition of the inner product on $\mathbb{R} X$, the set $\left\{a^{k} T: 0 \leq k \leq d-1\right\}$ is orthonormal. As $k$ runs over the indicated range, $\operatorname{cs}(r k / d)$ and $\operatorname{sn}(r k / d)$ run over the same values as each other, albeit in a different order. But $\operatorname{cs}^{2}(r k / d)+\operatorname{sn}^{2}(r k / d)=1$. Hence $\xi_{r}^{1}$ is a unit vector. The group element $a^{d / 4}$ acts on the Euclidian plane $e_{r} \mathbb{R} X$ as a rotation through $\pm \pi / 2$, so $\xi_{r}^{2}$ is a unit vector orthogonal to $\xi_{r}^{1}$. The claim is now established. A similar argument shows that $e_{r} \mathbb{R} Y$ has orthonormal basis $\left\{\eta_{r}^{1}, \eta_{r}^{2}\right\}$ where $\eta_{r}^{1}=2^{n / 2-1} e_{r} S$ and $\eta_{r}^{2}=a^{d / 4} \eta_{r}^{1}$.

For convenience, let us orientate the Euclidian planes $e_{r} \mathbb{R} X$ and $e_{r} \mathbb{R} Y$ such that $\xi_{r}^{1}$ and $\eta_{r}^{1}$ are due east of the origin, while $\xi_{r}^{2}$ and $\eta_{r}^{2}$ are due north. We measure angles anticlockwise from the east. Thus $a^{d / 4}$ acts on the two planes as the rotation through $\pi / 2$, so $a$ acts on the two planes as the rotation through an angle of $2 \pi \sigma_{r} r / d$ where $\sigma_{r}=(-1)^{(r-1) / 2}$. (That is, if $r \equiv 1 \bmod 4$, then $\sigma_{r}=1$; otherwise, $\sigma_{r}=-1$.) We now extend the notation. Let $\pm \mathscr{R}$ denote the set of odd integers $r$ in the range $1-d / 2 \leq r \leq d / 2-1$. We understand that $\chi_{-r}=\chi_{r}$ and $e_{-r}=e_{r}$. Still writing $\sigma_{r}=$ $(-1)^{(r-1) / 2}, \sigma_{-r}=-\sigma_{r}$ and the value of $2 \pi \sigma_{r} r / d$ remains unchanged when we replace $r$ with $-r$. So there is no ambiguity in our polar coordinates; for all $r \in \pm \mathscr{R}$, the group element $a$ still acts on the planes $e_{r} \mathbb{R} X=e_{-r} \mathbb{R} X$ and $e_{r} \mathbb{R} Y=e_{-r} \mathbb{R} Y$ as the rotation through an angle of $2 \pi \sigma_{r} r / d$.

The $D$-invariant isometry $l: \mathbb{R} Y \rightarrow \mathbb{R} X$ restricts to an isometry (1$\left.\sum_{r} e_{r}\right) \mathbb{R} Y \rightarrow\left(1-\sum_{r} e_{r}\right) \mathbb{R} X$ and to $d / 4$ isometries $l_{r}=l_{-r}: e_{r} \mathbb{R} Y=e_{-r} \mathbb{R} Y \rightarrow$ $e_{r} \mathbb{R} X=e_{-r} \mathbb{R} X$. When choosing $l$, all $1+d / 4$ of these $D$-invariant isometries can 
be chosen independently of one another. Since $e_{r} \mathbb{R} Y$ and $e_{r} \mathbb{R} X$ are absolutely irreducible, there are, as we noted above, precisely two choices for the isometry $l_{r}=$ $l_{-r}$. We shall be needing a formula for $l_{r}$ in terms of the polar coordinates that we have imposed.

The group element $s$ acts as a reflection on $e_{r} \mathbb{R} X$ and $e_{r} \mathbb{R} Y$. Plainly, $s$ fixes the line $\mathbb{R} \eta_{r}^{1}$ in $\mathbb{R} Y$. We have $s t=a^{-1}$, hence $s T=a^{-1} T$ and

$$
s \eta_{r}^{1}=a^{-1} \eta_{r}^{1}=\operatorname{cs}\left(-\sigma_{r} r / d\right) \eta_{r}^{1}+\operatorname{sn}\left(-\sigma_{r} r / d\right) \eta_{r}^{2}=\operatorname{cs}\left(\sigma_{r} r / d\right) \eta_{r}^{1}-\operatorname{sn}\left(\sigma_{r} r / d\right) \eta_{r}^{2} .
$$

So $s$ fixes the line $L_{r}=\mathbb{R}\left(\xi_{r}^{1}+s \xi_{r}^{1}\right)=\mathbb{R}\left(\operatorname{cs}\left(\sigma_{r} r / 2 d\right) \xi_{r}^{1}-\operatorname{sn}\left(\sigma_{r} r / 2 d\right) \xi_{r}^{2}\right)$. Being $D$ invariant, $l_{r}$ must send the $s$-fixed line $\mathbb{R} \eta_{r}^{1}$ to the $s$-fixed line $L_{r}$. But $l_{r}$ preserves unit vectors, and it also preserves the senses of the rotations $a^{k}$. Therefore, $l_{r}$ is given by

$$
l_{r}\left(\eta_{r}^{1}\right)=\operatorname{cs}\left(m_{r} / 2 d\right) \xi_{r}^{1}-\operatorname{sn}\left(m_{r} / 2 d\right) \xi_{r}^{2}, \quad l_{r}\left(\eta_{r}^{2}\right)=\operatorname{sn}\left(m_{r} / 2 d\right) \xi_{r}^{1}+\operatorname{cs}\left(m_{r} / 2 d\right) \xi_{r}^{2},
$$

where the integer $m_{r}=m_{-r}$ belongs to $\left\{\sigma_{r} r, \sigma_{r} r+d\right\}$. The equation for $l_{r}^{-1}$ is similar, with a change of \pm sign for the sn terms.

The case where $n=4$ and $r=3$ is illustrated in the diagram below. The lefthand half of the diagram depicts the plane $e_{3} \mathbb{R} Y$. Here, $a$ acts as a rotation through $2 \pi \sigma_{3} r / d=2 \pi \sigma_{3} 3 / 8$, and the sense $\sigma_{3}=-1$ is negative because the rotation $a^{d / 4}=a^{2}$ acts as a rotation through $\pi / 2$. The reflection $s$ fixes the $\eta_{1}$-axis. In the right-hand half of the diagram, $a$ again acts as a rotation through $-2 \pi 3 / 8$, but $s$ now fixes the line $L_{3}$. We can choose $l_{3}$ to be either one of the two rotations which sends the $\eta_{1}$-axis to the line $L_{3}$.
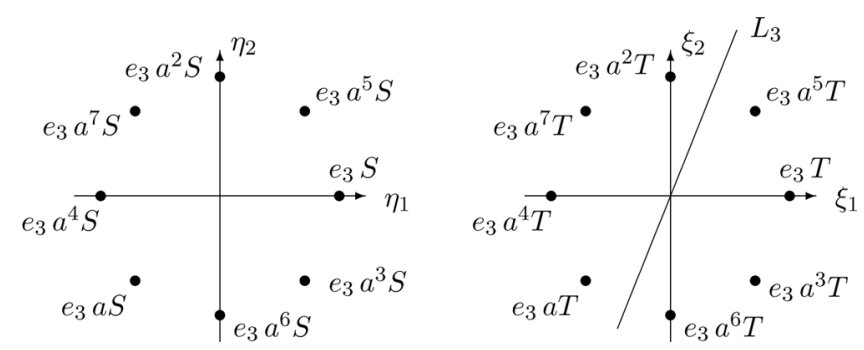

We have $\alpha(i)= \pm i$. For simplicity of discussion, let us assume that $\alpha(i)=i$; the argument in the other case is similar. There exists an integer $\ell$, well defined up to congruence modulo $2 d$, such that $\alpha(\epsilon)=\epsilon^{\ell}$ for all $2 d$ th roots of unity $\epsilon$. The condition $\alpha(\sqrt{2})=-\sqrt{2}$ implies that $\ell \equiv 3$ or $\ell \equiv 5 \bmod 8$. But the condition $\alpha(i)=i$ forces $\ell \equiv 5 \bmod 8$; indeed, $e^{i \pi / 4}=(1+i) / \sqrt{2}$, and hence $\alpha\left(e^{\pi i / 4}\right)=-e^{i \pi / 4}$. The condition $\alpha(i)=i$ also implies that $\alpha(\operatorname{cs}(c / 2 d))=\operatorname{cs}(\ell c / 2 d)$ and $\alpha(\operatorname{cs}(c / 2 d))=$ $\operatorname{cs}(\ell c / 2 d)$ for any integer $c$. By identifying $\pm \mathscr{R}$ with the multiplicative group of odd integers modulo $d$, we allow $\ell$ to act on $\pm \mathscr{R}$. Then $\alpha^{-1}\left(\xi_{r}^{1}\right)=\xi_{\ell^{-1} r}^{1}$. Since the actions of $\alpha$ and $a^{d / 4}$ commute, $\alpha^{-1}\left(\xi_{r}^{2}\right)=\xi_{\ell^{-1} r}^{2}$. Similarly, $\alpha\left(\eta_{r}^{1}\right)=\xi_{\ell r}^{1}$ and $\alpha\left(\eta_{r}^{2}\right)=\xi_{\ell r}^{2}$.

Using the above equations for the actions of $l^{ \pm 1}$ and $\alpha^{ \pm 1}$, a few lines of straightforward manipulation yield

$$
\theta\left(\xi_{r}^{1}\right)=\operatorname{cs}\left(n_{r} / 2 d\right) \xi_{r}^{1}+\operatorname{sn}\left(n_{r} / 2 d\right) \xi_{r}^{2}, \quad \theta\left(\xi_{r}^{1}\right)=-\operatorname{sn}\left(n_{r} / 2 d\right) \xi_{r}^{1}+\operatorname{cs}\left(n_{r} / 2 d\right) \xi_{r}^{2},
$$


where $n_{r}=\ell m_{\ell^{-1} r}-m_{r}$. We understand $n_{r}$ to be an integer determined by $l$ and $\alpha$ up to congruence modulo $2 d$. Note that $n_{r}=n_{-r}$. But $\chi_{r}$ occurs only once in $\mathbb{R} X$, and the equations for $\theta$ have real coefficients, so $\theta$ and $\theta_{\mathbb{R}}$ must restrict to the same operator on each $e_{r} \mathbb{R} X$. Moreover, this operator on $e_{r} \mathbb{R} X$ is $D$-invariant and orthogonal, so it must act as multiplication by \pm 1 . Therefore, $n_{r} \in\{0, d\}$ up to congruence modulo $2 d$, and we have $n_{r} \equiv d \bmod 2 d$ if and only if $\chi_{r}$ occurs in $W$.

Since $n_{r}=n_{-r}$ and since $\ell$ permutes the elements of $\pm \mathscr{R}$, we have

$$
\begin{aligned}
\sum_{r \in \mathscr{R}} n_{r} & =\frac{1}{2} \sum_{r \in \pm \mathscr{R}} n_{r} \equiv \frac{\ell-1}{2} \sum_{r \in \pm \mathscr{R}} m_{r}=(\ell-1) \sum_{r \in \mathscr{R}} m_{r} \\
& =(\ell-1)(1-3+5-7+\cdots+(d / 2-3)-(d / 2-1))=(1-\ell) d / 4
\end{aligned}
$$

modulo $2 d$. But $\ell \equiv 5 \bmod 8$, so $\sum_{r \in \mathscr{R}} n_{r} \equiv d \bmod 2 d$. The alternative proof of Corollary 11.5 is now complete.

The alternative proof shows something more. Let us confine our attention to the case $n=4$, that is, $D=D_{16}$. We understand the variables $r, m_{r}, n_{r}, \ell$ to be integers well defined up to congruence modulo $8,8,16,16$, respectively. Still assuming that $\alpha(i)=i$, there are eight possibilities, as indicated in Table 1 .

Changing the value of any one of the three variables $\ell, m_{1}, m_{3}$ changes the faithful irreducible character $\chi_{r}$ that occurs in $W$. In particular, we have another surprising result in the same vein as Corollary 11.5.

Proposition 12.1. Let $\alpha_{5}$ and $\alpha_{13}$ be automorphisms of $\mathbb{C}$ with the same Kummer symbols and such that $\alpha_{5}(\epsilon)=\epsilon^{5}$ and $\alpha_{13}(\epsilon)=\epsilon^{13}$ for any 16 th root of unity $\epsilon$. (Such automorphisms exist by Zorn's Lemma.) With the notation above, suppose that $n=4$. Fix a D-invariant isometry $\imath: \mathbb{R} Y \rightarrow \mathbb{R} X$. Let $W_{5}$ and $W_{13}$ be zombie modules for $\kappa_{4}$ constructed as above in the cases $\alpha=\alpha_{5}$ and $\alpha=\alpha_{13}$, respectively. Then the elements $\left[W_{5}\right]$ and $\left[W_{13}\right]$ of $A_{\mathbb{R}}(D)$ are not congruent modulo $2 A_{\mathbb{R}}(D)$.

We can now fulfill the promise that was made at the end of Section 6. In the proof of Lemma 8.1, we had to find a suitable E्E-submodule $U$ that was small enough to yield a certain desired conclusion, yet large enough to satisfy the hypothesis of Corollary $6.9, \alpha(\mathbb{C} U) \cong \mathbb{C} U$. If that hypothesis could be dropped then, instead of putting $U=U_{\chi}^{\mathrm{Gal}}$, we could put $U=U_{\chi}$, and the rest of the argument in Lemma 8.1 would imply that, fixing $X, Y$ and $\alpha$ then, up to parity, the multiplicity

Table 1

\begin{tabular}{lccccc}
\hline$\ell$ & $m_{1}=m_{7}$ & $m_{3}=m_{5}$ & $n_{1}=n_{7}$ & $n_{3}=n_{5}$ & {$\left[\left(e_{1}+e_{3}\right) W\right]$} \\
\hline 5 & 1 & 5 & 0 & 8 & $\chi_{3}=\chi_{5}$ \\
5 & 1 & 13 & 8 & 0 & $\chi_{1}=\chi_{7}$ \\
5 & 9 & 5 & 8 & 0 & $\chi_{1}=\chi_{7}$ \\
5 & 9 & 13 & 0 & 8 & $\chi_{3}=\chi_{5}$ \\
13 & 1 & 5 & 8 & 0 & $\chi_{1}=\chi_{7}$ \\
13 & 1 & 13 & 0 & 8 & $\chi_{3}=\chi_{5}$ \\
13 & 9 & 5 & 0 & 8 & $\chi_{3}=\chi_{5}$ \\
13 & 9 & 13 & 8 & 0 & $\chi_{1}=\chi_{7}$ \\
\hline
\end{tabular}


of $\chi$ in $W$ is independent of the choice of $\imath$. This conflicts with the data in the table above. In conclusion, the orientation behaviour of $\theta_{\mathbb{R}}$ at $\chi$ is entangled with the orientation behaviour of $\theta_{\mathbb{R}}$ at the Galois conjugates of $\chi$.

\section{REFERENCES}

[1] Barker, L. (2006). Genotypes of irreducible representations of finite p-groups. $J$. Algebra 306:655-681.

[2] Barker, L. (2008). Rhetorical biset functors, rational biset functors and their semisimplicity in characteristic zero. J. Algebra 319:3810-3853.

[3] Barker, L. (2010). Tornehave morphisms II: the lifted Tornehave morphism and the dual of the Burnside functor. J. Pure Appl. Algebra 214:1759-1777.

[4] Barker, L. Tornehave morphisms III: the reduced Tornehave morphism and the Burnside unit functor. Preprint.

[5] Boltje, R., Pfeiffer, G. (2007). An algorithm for the unit group of the Burnside ring of a finite group. Groups St. Andrews 2005 1:230-236 (LMS Lecture Notes Ser. 339, Cambridge Univ. Press, Cambridge).

[6] Bouc, S. (1996). Foncteurs d'ensembles munis d'une double action. J. Algebra 183:664736.

[7] Bouc, S. (2006). The Dade group of a p-group. Invent. Math. 164:189-231.

[8] Bouc, S. (2007). The functor of units of Burnside rings for p-groups. Comment. Math. Helv. 82:583-615.

[9] Bouc, S. (2008). Rational p-biset functors. Journal of Algebra 319:1776-1800.

[10] Bouc, S., Yalçın, E. (2007). Borel-Smith functions and the Dade group. J. Algebra 311:821-839.

[11] Bourbaki, N. (1989). Elements of Mathematics. Lie Groups and Lie Algebras. Chapters 1-3. Berlin: Springer.

[12] Tom Dieck, T. (1979). Transformation Groups and Representation Theory. Lecture Notes in Math. 766. Berlin: Springer.

[13] Tom Dieck, T. (1987). Transformation Groups Berlin: De Gruyter.

[14] Gluck, D. (1981). Idempotent formula for the Burnside algebra with applications to the $p$-subgroup simplical complex. Illinois J. Math. 25:63-67.

[15] Huppert, B. (1967). Endliche Gruppen, I. Berlin: Springer.

[16] Onishchik, A. L., Vinberg, E. B. (1990). Lie Groups and Algebraic Groups. Berlin: Springer.

[17] Tornehave, J. (1984). The Unit Theorem for the Burnside Ring of a 2-Group. Aarhaus Universitet, Preprint Series 1983/84, No. 41.

[18] Yalçın, E. (2005). An induction theorem for the unit groups of Burnside rings of 2-groups. J. Algebra 289:105-127.

[19] Yoshida, T. (1990). On the unit groups of Burnside rings. J. Math. Soc. Japan 42:31-64. 